\title{
Roles of Plant Growth-Promoting Rhizobacteria (PGPR) in Stimulating Salinity Stress Defense in Plants: A Review
}

\author{
Dung Minh Ha-Tran ${ }^{1,2,3} \mathbb{D}$, Trinh Thi My Nguyen ${ }^{2}$, Shih-Hsun Hung ${ }^{2,4} \mathbb{D}$, Eugene Huang ${ }^{5}$ \\ and Chieh-Chen Huang $2,6, *,+$
}

1 Molecular and Biological Agricultural Sciences Program, Taiwan International Graduate Program, Academia Sinica and National Chung Hsing University, Taipei 11529, Taiwan; hatranminhdung@gmail.com

2 Department of Life Sciences, National Chung Hsing University, Taichung 40227, Taiwan; mytrinhnguyen0410@gmail.com (T.T.M.N.); walter030170@gmail.com (S.-H.H.)

3 Graduate Institute of Biotechnology, National Chung Hsing University, Taichung 40227, Taiwan

4 Department of Horticulture, National Chung Hsing University, Taichung 40227, Taiwan

5 College of Agriculture and Natural Resources, National Chung Hsing University, Taichung 40227, Taiwan; eugenehuang@smail.nchu.edu.tw

6 Innovation and Development Center of Sustainable Agriculture, National Chung Hsing University, Taichung 40227, Taiwan

* Correspondence: cchuang@dragon.nchu.edu.tw

† Mailing Address: Life Sciences Building 4F. R.403, No.145, Xingda Rd., Taichung 402, Taiwan.

Citation: Ha-Tran, D.M.; Nguyen, T.T.M.; Hung, S.-H.; Huang, E.;

Huang, C.-C. Roles of Plant

Growth-Promoting Rhizobacteria (PGPR) in Stimulating Salinity Stress Defense in Plants: A Review. Int. J. Mol. Sci. 2021, 22, 3154. https:// doi.org/10.3390/ijms22063154

Academic Editor: Daniela Romano

Received: 28 February 2021

Accepted: 18 March 2021

Published: 19 March 2021

Publisher's Note: MDPI stays neutral with regard to jurisdictional claims in published maps and institutional affiliations.

Copyright: (c) 2021 by the authors. Licensee MDPI, Basel, Switzerland. This article is an open access article distributed under the terms and conditions of the Creative Commons Attribution (CC BY) license (https:// creativecommons.org/licenses/by/ $4.0 /)$.

\begin{abstract}
To date, soil salinity becomes a huge obstacle for food production worldwide since salt stress is one of the major factors limiting agricultural productivity. It is estimated that a significant loss of crops (20-50\%) would be due to drought and salinity. To embark upon this harsh situation, numerous strategies such as plant breeding, plant genetic engineering, and a large variety of agricultural practices including the applications of plant growth-promoting rhizobacteria (PGPR) and seed biopriming technique have been developed to improve plant defense system against salt stress, resulting in higher crop yields to meet human's increasing food demand in the future. In the present review, we update and discuss the advantageous roles of beneficial PGPR as green bioinoculants in mitigating the burden of high saline conditions on morphological parameters and on physiobiochemical attributes of plant crops via diverse mechanisms. In addition, the applications of PGPR as a useful tool in seed biopriming technique are also updated and discussed since this approach exhibits promising potentials in improving seed vigor, rapid seed germination, and seedling growth uniformity. Furthermore, the controversial findings regarding the fluctuation of antioxidants and osmolytes in PGPR-treated plants are also pointed out and discussed.
\end{abstract}

Keywords: PGPR; salt stress; salinity; abiotic stress; ACC deaminase; seed priming; IAA

\section{Introduction}

Soil salinization caused by saline irrigation regimes [1], by water scarcity [2], and by the rise in sea level due to global warming [3]. Another potential source causing soil salinity comes from compost fertilizer since the raw materials for composting operations are food waste and municipal organic waste that contain large quantities of $\mathrm{NaCl}$ [4]. Salinity not only hampers crop productivity, but also threatens the sustainability of agro-ecosystems worldwide. The osmotic stress caused by high salinity $(100-200 \mathrm{mM})$ is originated from the reduction in solute potential of soil solution. The reduced solute potential, in turn, leads to the decrease in hydraulic conductance and then in water and solute uptake by plants [5]. This conducts the prevalence of drought-like conditions and makes drought and salinity occur simultaneously in various agricultural systems [6]. Salinity stress also imposes nutrients deficiencies by interfering directly with ion transporters in the root plasma membrane (e.g., $\mathrm{K}^{+}$-selective ion channels) [7], and by inhibiting root growth [8-10]. Due to the rising severity of salinity on global food production, numerous strategies have been offered 
to cope with the increasing challenging soil conditions. Along with plant breeding [11], plant genetic engineering [12], and genetic transformation [13], agricultural practices have dramatically contributed to the improvement of plant tolerance to salinity stress. The supplement of calcium $\left(5 \mathrm{mM} \mathrm{CaCl}_{2}\right)$ ameliorated the reduction in shoot and root of salt-treated strawberry plants [14]. The pivotal physio-mechanical property of silicon (Si) has been widely noticed in most plants, especially its alleviating role in improving photosynthetic activity, enhancing essential nutrient uptake, and mitigating negative influence of abiotic stress [15]. In the study of Hassanvand et al. (2019) [16], the reduction of pigment content and essential oil yield in geranium (Pelargonium graveolens) plants caused by elevated EC levels was effectively ameliorated by a weekly $\mathrm{K}_{2} \mathrm{SiO}_{3}$ application. Green leaf volatiles (GLVs) are an important group of volatile organic compounds (VOCs) emitted by plants under stressful conditions [17], and enable plants to activate defense-related genes [18]. Z-3-hexeny-1-yl acetate (Z-3-HAC), a GLV, was used in seed priming to promote a better salt stress tolerance [17]. The Z-3-HAC-primed peanut (Arachis hypogaea L.) seedlings exhibited higher antioxidant enzymes (AEs) activities, a higher net photosynthetic rate (Pn), and an increased osmolyte accumulation, while reduced reactive oxygen species (ROS) levels, electrolyte leakage (EL), and lipid peroxidation (LP) as compared to the non-primed plants [17]. Being a metabolic intermediate in higher plants, 5-aminolevulinic acid (ALA) is a common precursor of tetrapyrroles such as chlorophyll (Chl), heme and siroheme, and this small signaling molecule also participates in several physiological processes to counteract salt stress damage [19]. In the study of Wu et al. (2018) [20], an exogenous application of ALA under salinity increased the contents of intermediates and Chl a, Chl b, as well as repaired the damages of photosynthetic apparatus. Melatonin (N-acetyl-5methoxytryptamine) (Mel), a ubiquitous multifunctional signaling molecule, functions as a stimulator in several physiochemical responses against stresses [21]. The application of exogenous Mel mitigated salt stress by increasing the contents of polyamines (PAs), the ubiquitous cellular components acting as antistress agents, in wheat seedlings [22]. Moreover, salt tolerance in Mel-treated rice plants was improved via the upregulation of $\mathrm{K}^{+}$transporter genes, the modulation of $\mathrm{K}^{+}$homeostasis and the scavenging of hydroxyl radicals [23].

The bacterization of plant crops with PGPR and the implementation of these useful rhizobacteria in seed biopriming have demonstrated their beneficial properties in enhancing plant growth and development, and in augmenting plant salt stress tolerance through different mechanisms. PGPR aid to alleviate salinity stress in plants by boosting water absorption capability, enhancing essential nutrients uptake, accumulating osmolytes (OS) (e.g., proline (Pro), glutamate (Glu), glycine betaine, soluble sugars, choline, O-sulphate, and polyols), increasing AEs activities (e.g., superoxide dismutase (SOD, EC 1.15.1.1), peroxidase (POD, EC 1.11.1.7), catalase (CAT, EC 1.11.1.6), ascorbate peroxidase (APX, EC 1.11.1.11), monodehydroascorbate reductase (MDAR, EC 1.6.5.4), dehydroascorbate reductase (DHAR, EC 1.8.5.1), glutathione reductase (GR, EC 1.6.4.2), and non-enzymatic antioxidants (NEAs) (e.g., ascorbate (ASC), glutathione (GSH), tocopherols (TCP), carotenoids (Car), and polyphenols (PPs)) in plant tissues [24-28]. In all types of salinity, sodium chloride $(\mathrm{NaCl})$ is the most soluble and widespread salt [17] and $\mathrm{Na}^{+}$is the primary cause of ion-specific damage for many plants, especially for graminaceous crops [29]. Consequently, to narrow down the scope of this review, we focus mainly on the negative effects of $\mathrm{Na}^{+}$ ion on plants, although high concentrations of $\mathrm{Cl}^{-}$anion are also toxic to plants. In this point of view, three terms "salt", "saline", and " $\mathrm{Na}^{+}$" were used interchangeably in the review to indicate the salinity. 


\section{Adverse Effects of Salinity on Plants}

\section{1. $\mathrm{Na}^{+}$Accumulation, Nutrients Uptake Inhibition, and Plant Growth Reduction}

Under salt stress, $\mathrm{Na}^{+}$is accumulated at higher concentrations in plant tissues, causing changes in $\mathrm{Na}^{+} / \mathrm{K}^{+}$ratio and the inhibition of essential nutrient uptake $[14,24,30]$. This could be attributed to the competition between similar ionic radii of $\mathrm{Na}^{+}$and $\mathrm{K}^{+}$in soils [31], causing the dysfunctional ionic selectivity of the cell membranes. In the review of Manishankar et al. [32], high $\mathrm{Na}^{+}$concentration in soil can change soil texture, leading to a decrease in soil porosity. This leads to the reduction of soil aeration and water conductance. Also according to Manishankar et al. [32], the zones of low water potential caused by high salt deposition in the soil make difficult for the roots to uptake water and nutrients. With $35 \mathrm{mM} \mathrm{NaCl}$ treatment, the $\mathrm{Na}^{+}$concentration in strawberry leaves and roots was 3.4-fold higher than that in the control plants [14]. Moreover, salt stress also caused the critical reduction in the fruit yield (FY) with 35\% yield loss in the variety Camarosa and $45 \%$ in the variety Oso Grande [14]. At $150 \mathrm{mM} \mathrm{NaCl}$, a tremendous increase (50.4-fold) in the $\mathrm{Na}^{+}$content, and an increase in the $\mathrm{Na}^{+} / \mathrm{K}^{+}$ratio $(1.48 \mathrm{vs.} 0.02)$ in the roots of Broussonetia papyrifera, a woody plant used in paper industry, was recorded, in harmony with the decrease in $\mathrm{K}^{+}(25.6 \%), \mathrm{Ca}^{2+}(23.3 \%), \mathrm{Mg}^{2+}(21.4 \%)$, and $\mathrm{P}^{3+}(8.4 \%)$ contents [33]. In contrast, an upsurge of $\mathrm{Na}^{+}$concentration was found in the leaves of canola plants (Brassica napus L.), with approximately 4 -fold greater than that in the roots [30]. The $\mathrm{Na}^{+}$content in the common bean leaves (Phaseolus vulgaris L.) was 5-7-fold higher than that in the control common bean leaves, whereas the $\mathrm{K}^{+}$content was decreased by $32-35 \%$ relative to the control plants [34]. Likewise, the $\mathrm{Na}^{+}$content in the salt treated-chickpea leaves (Cicer arietinum L.) was 3.2-fold higher than that in the control leaves, leading changes in $\mathrm{Na}^{+} / \mathrm{K}^{+}$ratio from 0.31 in the non-saline condition to 2.24 in the saline condition. The reduction in N, K, Ca, $\mathrm{Mg}$ contents was also recorded by $54 \%, 55 \%, 60 \%$, and $55 \%$, respectively as compared to those in the control leaves [35].

In general, phytotoxicity caused by high salt concentrations was found under in vitro and greenhouse conditions and the toxic symptoms increase correlatively with the increase in $\mathrm{NaCl}$ treatments. High salinity significantly affects plant growth and physio-biochemical aspects, resulting in the decrease in germination rate (GRA), fresh and dry matters, photosynthetic pigments, essential nutrients uptake, and most importantly, in the loss of final crop yields. In contrast, a significant increase in AEs activities, osmoregulators, LP, membrane damage, ROS contents, $\mathrm{Na}^{+}$accumulation, and $\mathrm{Na}^{+} / \mathrm{K}^{+}$ratio was obviously observed with the increasing $\mathrm{NaCl}$ concentrations [36]. The shoot dry weight (SDW) and root dry weight (RDW) of $35 \mathrm{mM} \mathrm{NaCl}$-treated strawberry plants (Fragaria $\mathrm{x}$ ananassa Duch) were $45.8 \%$ and $58.6 \%$ lower than those in the control plants, respectively [14]. Salt stress adversely hampers all stages of plant growth, causing the reduction in FY (227 vs. $415 \mathrm{~g} /$ plant), fruit weight (FW) (8.4 vs. $9.6 \mathrm{~g} /$ fruit), number of fruits per plant (NF) (27 vs. 43$)$, and water-soluble dry matter (SDM) (6.6\% vs. $8.4 \%)$ of stressed plants relative to the unstressed plants. The root dry weight (RDW) of common bean decreased by $59-61 \%$ and the final yield lost by $27-30 \%$ under $200 \mathrm{mM} \mathrm{NaCl}$ [34]. Similarly, at $200 \mathrm{mM} \mathrm{NaCl}$ concentration, salt stress reduced 38\% SDW and 50\% RDW of chickpea (Cicer arietinum cv. Giza 1) compared to the control plants [35]. Regarding the influence of salinity on nutritional values, although moderate saline stress enhanced glucosinolates and antioxidants contents in broccoli (Brassica oleracea L. var. italica cv. Marathon) $(40 \mathrm{mM} \mathrm{NaCl})$ [22], and the application of $6 \mathrm{dS} \mathrm{m}^{-1}(66 \mathrm{mM} \mathrm{NaCl})$ increased the contents of lycopene, $\beta$-carotene, vitamin $C$ and overall phenolic compounds (PCs) of tomato fruits [23], high salinity concentrations ( $15 \mathrm{dS} \mathrm{m}^{-1} \sim 200 \mathrm{mM}$ ) markedly reduced protein, fat, and crude fiber contents of wheat grains [24]. Moreover, the fruit size of tomato [37] and the FW of pepper [38], which are considered major determinants of price and marketable characteristics, were strongly reduced with the increase of saline levels. However, it is noteworthy that although high salt concentration affects plants in an adverse manner, the definition of "low", "moderate", or "high" salinity depends fundamentally on plant variety, growth stage, nutrient composition in soil, and irrigation regime, etc. 


\subsection{Impairment of Physio-Biochemical Attributes}

\subsubsection{Reduction in Photosynthetic Pigments}

Salinity stress causes an unrepairable damage to the photosynthetic apparatus at any development stage of plant's life as it alters the chloroplasts structure, degrades chloroplast envelope, and triggers chloroplast protrusions [39]. Numerous studies indicated that high salinity led to a serious degradation of Chl and Car in salt-stressed plants; however, the degrees of reduction in these photosynthetic pigments (PhoPs) depended largely on plant species, plant age, $\mathrm{NaCl}$ concentration and the duration of salt stress exposure. Specifically, only $9 \%, 11 \%, 13 \%$, and $14 \%$ reduction in the total chlorophyll (Tchl) were determined in the rice (Oryza sativa L.) [40], soybean (Glycine max (L.) Merr.) [41], maize (Zea mays L.) [42], and cucumber (Cucumis sativus L.) [43] seedlings, respectively. However, contrary to these studies, the reduced contents of Tchl were tremendously varied from $22 \%$ in oat (Avena sativa) seedlings [44], $41-42 \%$ in tomato (Solanum lycopersicum L.) seedlings [45,46], $44 \%$ in tomato (Solanum lycopersicum L.) plants [47], 50\% in peanut (Arachis hypogaea) seedlings [48], $56 \%$ in rice (Oryza sativa L.) seedlings [49] to 61\% in rapeseed (Brassica napus L.) plants [30], respectively. In addition, under salt detriment, $16 \%$ of Car decreased in the ginseng plantlets, $19 \%$ of Car reduced in the mung bean plants, and $49 \%$ of Car decreased in the tomato seedings were reported by Sukweenadhi et al. (2018) [50], Shahid et al. (2021) [36], and Akram et al. (2019) [45], respectively. In addition, $\mathrm{NaCl}$ toxicity also declined $\mathrm{Pn}$, stomatal conductance, and transpiration rate in the stressed plants [51].

\subsubsection{Increase in $\mathrm{LP}$}

Lipids are essential components of cell membranes responsible for structure maintenance and cell functions control [52]. ROS are generated from several life processes and an excess of ROS can damage cell, tissues and organs [53]. Salinity exposure brings about a disturbance, an overflow, or even a disruption of electron transport chains (ETC) in mitochondria and chloroplasts in higher plants, resulting in ROS accumulation [54]. The major site involved in the production of $\mathrm{O}_{2}{ }^{\bullet-}$ is the photosystem I (PSI). In the presence of light, $\mathrm{O}_{2}$ which is continuously provided by the water autolysis (Reaction $1: 2 \mathrm{H}_{2} \mathrm{O} \rightarrow 4 \mathrm{e}^{-}+\mathrm{O}_{2}$ $+4 \mathrm{H}^{+}$) can be reduced to $\mathrm{O}_{2}{ }^{\bullet-}$ (Reaction 2: $2 \mathrm{O}_{2}+2 \mathrm{e}^{-} \rightarrow 2 \mathrm{O}_{2}{ }^{\bullet-}$ ). The excess amount of reduced ferredoxin $\left(\mathrm{Fd}_{\mathrm{red}}\right)$ and the limited NADP availability induce the autoxidation of $\mathrm{Fd}_{\text {red }}$ to $\mathrm{Fd}_{\mathrm{ox}}$ and the generation of $\mathrm{O}_{2}{ }^{--}$(Reaction 3: $\mathrm{Fd}_{\text {red }}+\mathrm{O}_{2} \rightarrow \mathrm{Fd}_{\mathrm{ox}}+\mathrm{O}_{2}{ }^{\bullet-}$ ). In addition, the $\mathrm{Fd}_{\text {red }}$ can react with $\mathrm{O}_{2}{ }^{\bullet-}$ to form $\mathrm{H}_{2} \mathrm{O}_{2}$ (Reaction 4: $\mathrm{Fd}_{\text {red }}+\mathrm{O}_{2}{ }^{\bullet-}+2 \mathrm{H}^{+}$ $\rightarrow \mathrm{Fd}_{\mathrm{ox}}+\mathrm{H}_{2} \mathrm{O}_{2}$ ). Lipids are primary targets of ROS attack and the free radicals oxidation of polyunsaturated fatty acids is called LP [55]. As a byproduct of LP, malonaldehyde (MDA) has been largely used as an important indicator to evaluate the extent of damaging effects caused by ROS and oxidative stress combination on membrane lipids to reduce membrane stability [56]. The MDA content was tremendously increased by $36 \%$ in ginseng root plantlets [50], 39\% in maize [42], $47 \%$ in peanut [48], $70 \%$ in chickpea [35], $131 \%$ in oat [44], $153 \%$ in mung bean [36], and 300\% in rice seedlings [57], indicating an severe damage to cell membrane integrity and/or membrane permeability during salinity exposure [58].

\subsection{Increased Accumulation of ROS and Elevated Production of AEs, NEAs, and OS}

On the one hand, ROS function as signaling molecules to mediate a wide range of important biological processes during plant growth and development such as seed germination [59], cell differentiation [60], root primary growth [61], and stem cell activities [62]. On the other hand, an elevated accumulation of ROS in plant tissues also causes oxidative damage to protein, DNA, lipids, and Chl biosynthesis [63,64]. Salinity stress brings about excessive accumulations of ROS including superoxide radical $\left(\mathrm{O}_{2}{ }^{\bullet-}\right)$, hydrogen peroxide $\left(\mathrm{H}_{2} \mathrm{O}_{2}\right)$, singlet oxygen $\left({ }^{1} \mathrm{O}_{2}\right)$, and hydroxyl radicals $\left({ }^{\bullet} \mathrm{OH}\right)$, which disturb cellular redox homeostasis and lead to oxidative stress [65]. ROS homeostasis, therefore, is essential to maintain a delicate balance for plant growth, especially under environmentally adverse conditions. To deal with salinity-derived oxidative stress, plants possess enzymatic defense system that synthesizes an array of AEs, along with NEAs to neutralize and detoxify 
ROS [26,27]. The AEs conduct the scavenging activity by breaking down and removing free radicals, while the NEAs perform their scavenging functions by interrupting free radical chain reactions [66]. Furthermore, the accelerated synthesis and accumulation of OS are also the common responses executed by plants to provide osmotic adjustments and to protect cell membrane integrity [67]. In plants, Pro is synthesized by either glutamate pathway or orinithine pathway [68] and is accumulated in cytosol and vacuole under stressful conditions. Under non-stress conditions, Pro only accounts for less than $5 \%$ of the total pool of free amino acids in plants. However, under various stresses, the Pro concentration might increase up to $80 \%$ of the total amino acid pool, indicating its vital roles in ROS homeostasis and water balance in plants [69]. Pro was found to exhibit protective roles against damages caused by ${ }^{1} \mathrm{O}_{2}$ or ${ }^{\bullet} \mathrm{OH}$ [70]. Ethylene $\left(\mathrm{C}_{2} \mathrm{H}_{4}\right)$, a small volatile phytohormone in higher plants, is involved in all stages of plant growth and development, from seed germination to fruit ripening [71]. Furthermore, ethylene has been considered as a stress hormone since it participates in plant responses to various types of stress such as wounding [72], salinity [73], and drought [74]. Although a small amount of ethylene, which is immediately produced after the onset of a stress, can initiate the systemic resistance in plants, the excess amount of ethylene from the second peak could bring about the inhibition of plant growth or even lead to cell death [75].

\section{Plant Growth-Promoting Rhizobacteria as the Promising Bioinoculants for Plant Crops}

\subsection{Key Criteria for Being Applicable PGPR}

The close alliance among soil, plant, and microbes exists during the entire life cycle of plants promotes plant development, induces systemic resistance in the host plant against pathogens and mitigates salinity stress [76]. PGPR have been widely used for decades to control insects pests [77], plant diseases [78], to promote plant growth [79], to manage nutrient [80], and to alleviate abiotic stress [81]. The ameliorative functions of PGPR consist of three aspects, namely, the ability to protect themselves against hyperosmotic conditions and abnormal $\mathrm{NaCl}$ concentrations, the capacity to aid plant tolerate better to elevated salinity, and to improve soil quality [82]. Regarding the alleviating roles of PGPR in promoting plant salinity tolerance, PGPR exhibit beneficial traits in mitigating the toxic effects of high salt concentrations on morphological, physiological, and biochemical processes in plants, resulting in the significant rescue of yield loss. According to Fouda et al. [83], the application of PGPR could ameliorate the negative impacts of salinity via two main mechanisms as follows: (1) PGPR activate stress response systems in the host plants soon after the exposure of the plants to salinity, and (2) PGPR synthesize antistress biochemicals such as AEs, NEAs, and OS that are responsible for the removal of ROS [84]. Furthermore, PGPR can also mitigate salt stress symptoms by producing $\mathrm{Na}^{+}-$ binding exopolysaccharides (EPS), improving ion homeostasis, decreasing ethylene levels through enzyme 1-aminocyclopropane-1-carboxylate (ACC) deaminase, and synthesizing phytohormones [85-87].

\subsubsection{ACC Deaminase-Producing PGPR and Other Plant Growth Promoting Attributes}

Enzyme ACC deaminase [EC 4.1.99.4] catalyzes the cleavage of 1-aminocyclopropane1-carboxylate (ACC), an intermediate precursor of ethylene in higher plants, to produce $\alpha$-ketobutyrate and ammonia [88]. A proper amount of ethylene derived from the existing pool of ACC, or so called the small peak of ethylene in the biphasic ethylene response model described by Glick et al. [89] and Pierik et al. [90], is thought to be useful to plants in activating plant defensive responses to stress stimuli (e.g., temperature extremes, drought or flooding, insect pest damages, phytopathogens, and mechanical wounding) [91]. However, an elevated ethylene accumulation, also called stress ethylene or the larger peak of ethylene in the biphasic model, may cause harmful effects (e.g., chlorosis, abscission, and senescence) on plant growth [92], even lead to dead when present at high concentrations in plant tissues [93]. Although PGPR possess many different mechanisms to maintain plant growth 
under salinity detriment, the production of ACC deaminase is extremely important in reducing the elevated levels of ethylene, thereby indirectly support plant growth. The ACC deaminase-producing PGPR that live on plant surfaces or colonize in the plant tissues function as a sink for ACC [30] and the use of ACC as a nitrogen (N) source is beneficial to plant health since $\mathrm{N}$ uptake is always suppressed under salt conditions [94]. Up to now, a plethora of PGPR that have been studied to evaluate their roles in mitigating salinity stress in plants. The PGPR, namely, Pseudomonas putida UW4 [30], Arthrobacter protophormiae [95], Enterobacter sp. EJ01 [96], Enterobacter sp. UPMR18 [97], Zhihengliuella halotolerans, Bacillus gibsonii, Halomonas sp. [98], Chryseobacterium gleum sp. SUK [99], Pseudomonas fluorescens 002 [100], Microbacterium oleivorans KNUC7074, Brevibacterium iodinum KNUC7183, and Rhizobium massiliae KNUC7586 [101], Stenotrophomonas maltophilia SBP-9 [102], Enterobacter sp. P23 [49], Burkholderia sp. MTCC 12259 [57], Paenibacillus yonginensis DCY84 [50], Bacillus pumilus strain FAB10 [51], Pantoea agglomerans [103], Aneurinibacillus aneurinilyticus and Paenibacillus sp. [88], Leclercia adecarboxylata MO1 [104], Pseudomonas argentinensis and Pseudomonas azotoformans [105], Bacillus subtilis (NBRI 28B), B. subtilis (NBRI 33 N), Bacillus safensis (NBRI 12 M) [106], Bacillus megaterium NRCB001, B. subtilis subsp. subtilis NRCB002, B. subtilis NRCB003 [107], and Kosakonia sacchari [36] can produce ACC deaminase, as well as other important products such as indole-3-acetic acid (IAA), siderophore (Sid), EPS, and Pro. In addition, PGPR can conduct biofilm forming, N fixation, phosphate (P) solubilization, hydrogen cyanide $(\mathrm{HCN})$ and antifungal enzymes production [99]. The capability of PGPR for moderating salinity damage could be considered an indispensable trait for strain selection [108], reflecting in the elevated amounts of ACC deaminase, IAA, EPS, GSH, and Pro produced by themselves during salt exposure to protect their cells against the damaging effects of high $\mathrm{NaCl}$ concentrations. For instance, at $500 \mathrm{mM} \mathrm{NaCl}$, Sphingomonas sp. LK11 produced more GSH and Pro to counteract the detrimental effects of salinity imposed on its growth [108]. Similarly, the productions of ACC deaminase and Pro by the halotolerant Burkholderia sp. MTCC 12259 were highly correlated with the increasing $\mathrm{NaCl}$ concentrations in the medium broth, in which ACC deaminase reached the highest at $600 \mathrm{mM} \mathrm{NaCl}$, while the highest Pro level was obtained at $1000 \mathrm{mM} \mathrm{NaCl}$ [57]. This result was in accordance with the report of Ilyas et al. [109] when the Pro produced by a consortium consisting of Bacillus sp. (KF719179), Azospirillum brasilense (KJ194586), Azospirillum lipoferum (KJ434039), and Pseudomonas stutzeri (KJ685889) reached the maximum value at the highest $\mathrm{NaCl}$ concentration $(10 \%, w / v)$. Also, the productions of ROS-quenching enzymes SOD, CAT, POD, PPO, and Pro in Enterobacter sp. P23 were increased with the increase in $\mathrm{NaCl}$ concentrations [49]. The levels of IAA, Sid, and ACC deaminase produced by $\mathrm{K}$. sacchari strain MSK1 were increased with the increasing $\mathrm{NaCl}$ concentrations and reached the highest levels at the highest $\mathrm{NaCl}$ concentration $(400 \mathrm{mM}$ ) [36]. Recently, Misra and Chauhan [106] found that two B. subtilis strains NBRI 28B, NBRI 33N, and B. safensis NBRI $12 \mathrm{M}$ increased the production of ACC deaminase, biofilm, EPS, and Alginate (Alg) in proportion to the increasing $\mathrm{NaCl}$ concentrations in nutrient broth. This finding was in corroboration with the previous study of Mukherjee et al. [110], in which Halomonas sp. Exo1 could tolerate up to $20 \%(w / v)$ salt concentration and its EPS yield was directly proportional to the increasing $\mathrm{NaCl}$. These findings indicate that to be selected as potential bioinoculants for improving crop yield in saline soil, the PGPR candidates need to possess an ability to withstand and respond appropriately to high salinity in the environment.

3.1.2. Improvements of Growth Parameters, Nutrients Uptake, and Photosynthetic Pigments in PGPR-Inoculated Plants under Non-Stress Conditions

The halotolerant bacterium Enterobacter sp. strain P23 isolated from India's rice fields possesses the abilities to exhibit high ACC deaminase activity, to solubilize $\mathrm{P}$, to produce IAA, Sid, and HCN [49]. In non-tress conditions, the P23-inoculated rice seedlings showed better morphological parameters, namely shoot length (SL), root length (RL), shoot fresh weight (SFW), SDW, root fresh weight (RFW), and RDW, higher Chl content than those in the non-inoculated rice seedlings. This result was consistent with numerous other studies where the PGPR-inoculated plants grew better than the non-inoculated plants in 
normal environments. Specifically, the values of SFW, RFW, SDW, RDW, Chl a, Chl b, Car, and N, $\mathrm{P}$, and $\mathrm{K}$ concentrations in the S20-inoculated maize seedlings were increased by $2 \%, 6 \%, 5 \%, 2 \%, 4 \%, 7 \%, 2 \%, 16 \%, 43 \%$, and $2 \%$, respectively, as compared to the control seedlings [111]. Also, in the Chryseobacterium gleum sp. SUK + feather lysate inoculum (FLI)-inoculated wheat seedlings, an increase in $24 \%$ Tchl, and in $13 \%$ amino acids was noticed [99]. Likewise, an increase in SL, RL, SFW, RFW, and Tchl was observed in the L. adecarboxylata-inoculated tomato plants with $22 \%, 16 \%, 28 \%, 51 \%$, and $13 \%$ higher than those in the control plants, respectively [104]. The same trend in increased vegetative parameters was found in the studies of Li and Jiang [42], Khan et al. [40], Sapre et al. [44], Sarkar et al. [49], Akram et al. [45], and Alexander et al. [48]. The increase in Tchl was widely observed in various studies, however, the extent to which these pigments increased depends on PGPR strains, $\mathrm{NaCl}$ treatments, and plant species. For instance, only a $5 \%$ Chl increase in maize seedling bacterized with B. aquimaris DY-3 was noticed by Li and Jiang (2017) [42], whereas a $12 \%$ increase in P. putida H-2-3-inoculated soybean seedlings [41], a 17\% increase in S. maltophilia BJ01-peanut seedlings [48], a 29\% increase in K. sacchari-treated mung bean seedlings [36], a 41\% increase in Bacillus megaterium BMA12bacterized tomato seedlings [45], 46\% in B. pupilus-inoculated rice seedlings [40], and 60\% in A. brasilense-treated white clover plants [58].

PGPR can change root-system architecture by producing phytohormones, especially auxins (Aux) [112], volatile compounds [113,114], and by mediating plant ethylene levels via enzyme ACC deaminase [115]. The inoculation of Arabidopsis plants with Bacillus megaterium caused a suppression in primary root growth, while induced lateral root growth development, increased lateral root number, and promoted root hair length [116]. Recently, the research group of Chu et al. (2020) [117] also found that Pseudomonas PS01 inhibited the elongation of primary roots and triggered the formation of lateral root and the development of root hair. López-Bucio and colleagues [116] suggested that the inhibition of primary root was caused by a decrease in cell elongation and by a reduced cell proliferation in the root meristem. Vegetative parameters of the endophytes-inoculated sorghum plants (Sorghum bicolor) were widely varied with different endophytic PGPR species [118]. Intriguingly, although the amounts of IAA produced by Pseudomonas plecoglossicida-R382, Serratia marcescens-R381, Pantoea coffeiphila-R342, Bacillus cereus-R8, Rhodopseudomonas boonkerdiiR102, and Nocardioides aromaticivorans-R21 were comparable, the RDWs of their respective inoculated sorghum plants were significantly different [118]. This finding suggests that besides the effects of the bacterial IAA on root plant architecture, the interactions between plant and microbe are multifaceted and might play a major role in shaping root system development [119].

The positive influences of PGPR treatment on fruit/grain quality, total yield, and marketable grade yield were also investigated. The FY, fruit marketable yield (FMY), FW, fruit length (FL), fruit diameter (FD), and texture of red fruit in Bacillus subtilis BEB-13bsinoculated tomato plants were improved by $21 \%, 6 \%, 29 \%, 9 \%$, and $5 \%$, respectively in comparison with the control plants [120]. The maximum grain yield was recorded in the wheat plants treated with a triple combination of Bacillus megaterium, Enterobacter sp. and Arthrobacter chlorophenolicus [121], as well as the highest nutrient contents (e.g., N, P, $\mathrm{Cu}, \mathrm{Zn}, \mathrm{Mn}$, and $\mathrm{Fe}$ ) were observed in the treated wheat grains.

Nevertheless, in some exception cases, the applications of PGPR under normal conditions did not promote plant growth and yield. The PGPR even exhibited negative effects on the growth of eggplant and tomato plants as reported in the studies of Abd El-Azeem et al. [24] and Vaishnav et al., respectively [47]. Specifically, the SFW, SDW, and yield of eggplant were decreased by $8 \%, 9 \%, 12 \%$, respectively after inoculated with $X$. autotrophicus BM13, decreased by $12 \%, 21 \%$, and $30 \%$, respectively when inoculated with Bacillus brevis FK2 [24], as well as the SL of Sphingobacterium BHU-AV3-inoculated tomato was reduced by $11 \%$ [47]. Similarly, the SL, RL, and total plant fresh weight (TPFW) of C. gleum-inoculated wheat plants were decreased by $16 \%, 36 \%$, and $13 \%$, respectively relative to the control [99]. The data in these previous reports were in accordance with our 
preliminary data (unpublished data) as the SFW and RFW values of the Curtobacterium sp. C1-inoculated Arabidopsis plants were lower than those in the uninoculated plants.

Although the suppressive impacts of PGPR on plant growth and yield are scarcely recorded under non-stress conditions, this should be taken into consideration prior to PGPR bacterization practices in field. Furthermore, the response of plant variety to PGPR is genotype-dependent as shown in the report of Nawaz et al. [122] where the salt tolerant wheat genotype Aas-11 responded positively to Bacillus pumilus and Exiguobacterium aurantiacum, whereas the salt sensitive wheat genotype Galaxy-13 responded better to Pseudomonas fluorescence. In this regard, we should agree that the interactions between host plants and microbes are complicated and not always a win-win situation. In addition, the adaptation of plant species to PGPR might markedly vary from case to case due to genetic variation. More investigations at molecular level are required to deeply elucidate the multi-dimensional impacts of microbes on plants.

\subsubsection{Improvements of Growth Parameters, Nutrients Uptake, and Photosynthesis in} PGPR-Inoculated Plants under Salinity Conditions

Although PGPR can promote plant growth and improve nutrients uptake, as well as stimulate the synthesis of PhoPs in non-stress environments, their ameliorative roles in plant defense responses are fully expressed till plant crops endure harsh environmental conditions. In the reports of Awad et al. [123] and Abd El-Ghany and Attia [124], they found that the bacterization of maize (Zea mays L.) plants and faba bean (Vicia faba cv. Giza3) seeds with Azotobacter chroococcum, an EPS-producing bacterium, had the decreased $\mathrm{Na}^{+}$and $\mathrm{Cl}^{-}$concentrations and the increased $\mathrm{N}, \mathrm{P}$, and $\mathrm{K}$ concentrations in their plant tissues. PPs, known as potent antioxidants, can eliminate radical species (e.g., ${ }^{1} \mathrm{O}_{2}$, $\mathrm{O}_{2}{ }^{\bullet-}, \mathrm{OH}^{-}, \mathrm{H}_{2} \mathrm{O}_{2}$ ), thus preventing the propagation of oxidative chain reactions [125]. In the study of Hichem et al. [126], the amounts of total PPs including phenolic acids, flavonoids, anthocyanins and proanthocyanidins increased accordingly with the increased salinity in young and mature maize leaves and the elevated concentrations of these PCs had an inverse correlation with $\mathrm{H}_{2} \mathrm{O}_{2}$ content and LP level in leaves, indicating the scavenging activity of endogenous PCs against free radicals [127]. The total PPs in the leaves of Azotobacter chroococcum-inoculated maize seedlings were always higher than those in the non-inoculated maize seedlings, regardless of salt concentrations [128]. Moreover, the total PPs reached the highest level at the highest $\mathrm{NaCl}$ treatment $(5.85 \mathrm{~g} \mathrm{NaCl} / \mathrm{kg}$ soil). Abd_Allah et al. [35], who evaluated the effects of endophytic B. subtilis (BERA71) on mitigating saline soil stress in chickpea plants (Cicer arietinum cv. Giza 1), found that the B. subtilis (BERA71)-inoculated chickpea plants yielded higher plant biomass, achieved higher photosynthetic pigments, while reduced ROS levels, and LP compared to the non-inoculated seedlings. The positive correlation between Pro accumulation and salt stress adaptation has been widely recognized. However, the results are still controversial, and more investigations should be conducted to thoroughly explain the underlying mechanisms that regulate AEs and OS production.

Regarding nutrient acquisition, the PGPR helped to decrease $\mathrm{Na}^{+}$accumulation, whereas enhanced the acquisition of $\mathrm{N}, \mathrm{Ca}, \mathrm{Mg}$, and $\mathrm{K}$ contents in the chickpea plants [35]. The increased uptake of $\mathrm{Mg}^{2+}$ induced by Bacillus subtilis and Bacillus pumilus inoculation was associated with the elevated PhoPs contents since $\mathrm{Mg}^{2+}$ is the major component of $\mathrm{Chl}[40,129]$. Accordingly, the expression level of $\mathrm{Cab2}$, the gene encoding a $\mathrm{Chl} \mathrm{a} / \mathrm{b}$ protein in Arabidopsis plant, was downregulated in Mg-deficient plants before any obvious symptom of chlorophyll deficiency appears [130]. However, Abd_Allah and his colleagues [35] did not investigate the mechanisms that enhanced the uptake of essential nutrients. Therefore, it is unclear whether the increased nutrient acquisition in the B. subtilis-inoculated chickpea plants was due to the modulation of root architecture $[117,131]$, the mobilization of $\mathrm{P}$ in the soil $[132,133]$, or the N fixation $[134,135]$ induced by B. subtilis. Similarly, Khan et al. [40] noticed a limited uptake of $\mathrm{Na}^{+}$in B. pumilus-inoculated paddy plants, but the fundamental mechanism that suppressed $\mathrm{Na}^{+}$uptake was not thoroughly investigated yet. In contrast, an extensive accumulation of $\mathrm{Na}^{+}$was observed in the shoots of 
Bacillus-inoculated halophyte Arthrocnemum macrostachyum under high $\mathrm{NaCl}$ concentration $(1030 \mathrm{mM})$ [136]. Up to now, a plenty of studies recognize the roles of PGPR in increasing $\mathrm{K}^{+} / \mathrm{Na}^{+}$ratio, in activating $\mathrm{K}^{+}-\mathrm{Na}^{+}$selectivity, in maintaining PhoPs, in enhancing nutrient uptakes, thereby alleviating salt stress in saline environments [40]. However, more studies are needed to clearly elucidate the mechanisms underlying these phenomena. The key findings in recent PGPR studies were presented in Table 1.

3.1.4. Improvements of Growth Parameters, Nutrients Uptake, and Photosynthesis in PGPR-Primed Seeds and Their Respective Seedlings under Salinity Conditions

Seed is a dramatically important component of agricultural production since it is considered the primary determinant in establishing a fruitful crop. Moreover, seed germination is the first and the most critical stages of the plant's life cycle $[137,138]$. The uniformity of seed germination is one of the fundamental criteria that is used to evaluate SV [139]. In the era of climate change, seeds always suffer from the environmental challenges that may cause the reduction in seed GRA, GP, and the dysfunction of seedlings, resulting in a decrease in ultimate crop yields. Germinating seeds and seedlings appear to be more sensible to salinity than the growing plants since the germination stage occurs on saline soil surface where the drought-like condition reduces SV, suppresses protein synthesis, and disturbs structural organization in germinating embryos $[140,141]$. In addition, seed germination is strongly associated with the seedlings survival rate, as well as the subsequent vegetative growth [142]. $\alpha$-amylase is a key player in starch hydrolysis during seed germination since it supplies carbon source and energy to germinating seeds in the early stages of development before the initiation of the photosynthetic machinery [137]. A reduced water uptake and a decrease in $\alpha$-amylase activity caused by $\mathrm{NaCl}$ might cause the delay of germination time [143]. Furthermore, the data from Dehnavi et al. (2020) [138] demonstrated that salinity accounted for $98 \%$ of the variation in tested parameters including GP, germination index, mean germination time, SVI, SL, and RL of seedlings, fresh and dry weight of seedlings, and salinity tolerance indices.

Seed biopriming with living PGPR inoculum stimulates a speed and an uniformity of gemination, assures a rapid, uniform, and high establishment of crops, thereby improving yield and fruit/grain quality in non-stress and harsh conditions [144]. Under non-stress conditions, the GRAs of two endangered fir plant species Abies hickelii and Abies religiosa were highly stimulated by a combination of 12 h-hydropriming with PGPR biopriming, resulting an improved GRA up to $91 \%$ of $P$. fluorescens JUV8-primed $A$. hickelli seeds vs. $28 \%$ of unprimed control and up to $68 \%$ of $B$. subtilis BsUV-primed $A$. religiosa seeds vs. $32 \%$ of unprimed control [145]. Similarly, the GRA of isolate Ac26-primed wheat seeds was increased to $93.3 \%$ and the vigor index was 2830.7, much higher than those of the unprimed control with $53.3 \%$ and 1097.5 , respectively [146]. The subsequent development of primed plants was also better than the unprimed plants, suggesting the lasting impacts of PGPR treatment on physio-biochemical attributes of the treated plants $[145,146]$. 
Table 1. Ameliorative effects of plant growth-promoting rhizobacteria (PGPR) on plant growth and physio-biochemical parameters under salinity conditions.

\begin{tabular}{|c|c|c|c|c|c|c|c|c|c|c|}
\hline PGPR & Treatments & GP & Hormones & PhoPs & MDA & AEs & NEAs & Pro & Ion Content & Sources \\
\hline \multirow{5}{*}{$\begin{array}{l}\text { B. cepacia SE4, } \\
\text { Promicromonospora } \\
\text { SE188, } \\
\text { and A. calcoaceticus } \\
\text { SE370. } \\
\text { 7-day-old tomato } \\
\text { seedlings inoculated } \\
\text { with PGPR. }\end{array}$} & Control & & & & & & & & & [43] \\
\hline & $\begin{array}{l}120 \mathrm{mM} \mathrm{NaCl}+ \\
\text { Uninoculated }\end{array}$ & $\begin{array}{l}\downarrow 17 \% \text { SFW } \\
\downarrow 25 \% \text { SDW }\end{array}$ & $\begin{array}{l}\uparrow 255 \% \\
\mathrm{ABA}, \\
\uparrow 194 \% \mathrm{SA}\end{array}$ & $\downarrow 14 \%$ Tchl & \multirow{4}{*}{ N/A } & $\begin{array}{l}\uparrow 86 \% \text { CAT } \\
\uparrow 213 \% \text { POD } \\
\uparrow 456 \% \text { PPO }\end{array}$ & $\uparrow 79 \% \mathrm{PP}$ & \multirow{4}{*}{$\mathrm{N} / \mathrm{A}$} & $\begin{array}{l}\text { In shoot: } \\
\uparrow 740 \% \mathrm{Na}^{+} \\
\downarrow 4 \% \mathrm{~K}^{+} \\
\mathrm{Na}^{+} / \mathrm{K}^{+} \text {ratio } \sim 0.28\end{array}$ & \\
\hline & $\underset{\mathrm{SE} 4}{120 \mathrm{mM} \mathrm{NaCl}}+$ & $\begin{array}{l}\downarrow 11 \% \text { SFW } \\
\downarrow 8 \% \text { SDW }\end{array}$ & $\begin{array}{l}\uparrow 10 \% \mathrm{ABA} \\
\uparrow 367 \% \mathrm{SA}\end{array}$ & $\downarrow 0 \%$ Tchl & & $\begin{array}{l}\uparrow 27 \% \text { CAT } \\
\uparrow 163 \% \text { POD } \\
\uparrow 333 \% \text { PPO }\end{array}$ & $\uparrow 45 \% \mathrm{PP}$ & & $\begin{array}{l}\text { In shoot: } \\
\uparrow 297 \% \mathrm{Na}^{+}\end{array}$ & \\
\hline & $\begin{array}{c}120 \mathrm{mM} \mathrm{NaCl}+ \\
\text { SE118 }\end{array}$ & $\begin{array}{l}\downarrow 13 \% \mathrm{SFW} \\
\downarrow 6 \% \mathrm{SDW}\end{array}$ & $\begin{array}{l}\uparrow 6 \% \mathrm{ABA} \\
\uparrow 217 \% \mathrm{SA}\end{array}$ & $\downarrow 0 \%$ Tchl & & $\begin{array}{l}\uparrow 23 \% \text { CAT } \\
\uparrow 131 \% \text { POD } \\
\uparrow 322 \% \text { PPO }\end{array}$ & $\uparrow 35 \% \mathrm{PP}$ & & $\mathrm{Na}^{+} / \mathrm{K}^{+}$ratio $\sim 0.11$ & \\
\hline & $\begin{array}{l}120 \mathrm{mM} \mathrm{NaCl}+ \\
\text { SE370 }\end{array}$ & $\begin{array}{l}\downarrow 10 \% \text { SFW } \\
\downarrow 9 \% \text { SDW }\end{array}$ & $\begin{array}{l}\uparrow 23 \% \mathrm{ABA}, \\
\uparrow 261 \% \mathrm{SA}\end{array}$ & $\downarrow 0 \%$ Tchl & & $\begin{array}{l}\uparrow 46 \% \text { CAT } \\
\uparrow 156 \% \text { POD } \\
\uparrow 322 \% \text { PPO }\end{array}$ & $\uparrow 52 \% \mathrm{PP}$ & & & \\
\hline \multirow{4}{*}{$\begin{array}{l}\text { P. putida } \mathrm{H}-2-3 \text {. } \\
\text { 21-day-old soybean } \\
\text { seedlings inoculated } \\
\text { with P. putida. }\end{array}$} & Control & & & & & & & & & [41] \\
\hline & $\begin{array}{l}120 \mathrm{mM} \mathrm{NaCl}+ \\
\text { Uninoculated }\end{array}$ & $\begin{array}{l}\downarrow 18 \% \text { SL } \\
\downarrow 12 \% \text { TPFW }\end{array}$ & $\begin{array}{l}\uparrow 33 \% \mathrm{ABA} \\
\uparrow 114 \% \mathrm{SA} \\
\downarrow 11 \% \mathrm{JA}\end{array}$ & $\downarrow 11 \%$ Tchl & \multirow{3}{*}{ N/A } & $\uparrow 301 \%$ SOD & $\begin{array}{l}\downarrow 23 \% \\
\text { total PP }\end{array}$ & \multirow{3}{*}{$\mathrm{N} / \mathrm{A}$} & $\begin{array}{l}\text { In whole plant: } \uparrow 86 \% \mathrm{Na}^{+}, \uparrow \\
55 \% \mathrm{P}\end{array}$ & \\
\hline & $\begin{array}{c}0 \mathrm{mM} \mathrm{NaCl} \\
\text { putida }\end{array}$ & $\begin{array}{l}\uparrow 17 \% \text { SL } \\
\uparrow 8 \% \text { TPFW }\end{array}$ & $\begin{array}{l}\uparrow 18 \% \mathrm{ABA} \\
\uparrow 29 \% \mathrm{SA} \\
\downarrow 25 \% \mathrm{JA}\end{array}$ & $\uparrow 12 \%$ Tchl & & $\uparrow 2 \% \mathrm{SOD}$ & $\begin{array}{l}\text { Unchanged } \\
\text { total PP }\end{array}$ & & $\begin{array}{l}\text { In whole plant: } \downarrow 17 \% \mathrm{Na}^{+}, \uparrow \\
22 \% \mathrm{P}\end{array}$ & \\
\hline & $\begin{array}{l}120 \mathrm{mM} \mathrm{NaCl}+ \\
\text { P. putida }\end{array}$ & $\begin{array}{l}\downarrow 9 \% \text { SL } \\
\downarrow 0 \% \mathrm{TPFW}\end{array}$ & $\begin{array}{l}\downarrow 6 \% \mathrm{ABA} \\
\downarrow 26 \% \mathrm{SA} \\
\uparrow 54 \% \mathrm{JA}\end{array}$ & $\downarrow 7 \%$ Tchl & & $\uparrow 4 \% \mathrm{SOD}$ & $\begin{array}{l}\uparrow 4 \% \\
\text { total PP }\end{array}$ & & $\begin{array}{l}\text { In whole plant: } \uparrow 45 \% \mathrm{Na}^{+}, \uparrow \\
30 \% \mathrm{P}\end{array}$ & \\
\hline \multirow{3}{*}{$\begin{array}{l}\text { B. pumilus. } \\
\text { 14-day-old rice } \\
\text { seedlings inoculated } \\
\text { with B. pumilus. }\end{array}$} & Control & & & & & & & & & [40] \\
\hline & $\underset{\text { pumilus }}{0 \mathrm{mM} \mathrm{NaCl}}+B$ & $\uparrow 22 \%$ SFW & \multirow{2}{*}{ N/A } & $\uparrow 46 \%$ Tchl & \multirow{2}{*}{$\mathrm{N} / \mathrm{A}$} & $\begin{array}{l}\uparrow 22 \% \text { SOD } \\
\uparrow 20 \% \text { POD } \\
\uparrow 73 \% \text { CAT }\end{array}$ & \multirow{2}{*}{ N/A } & $\uparrow 7 \%$ & $\begin{array}{l}\text { In shoot: } \downarrow 54 \% \mathrm{Na}^{+}, \\
\uparrow 57 \% \mathrm{~K}^{+}, \uparrow 76 \% \mathrm{Mg}^{2+}, \\
\uparrow 18 \% \mathrm{Ca}^{2+}, \mathrm{Na}^{+} / \mathrm{K}^{+} \text {ratio } 0.27\end{array}$ & \\
\hline & $\begin{array}{l}10 \text { ppm Boron + } \\
\text { Uninoculated }\end{array}$ & $\downarrow 0 \% \mathrm{SFW}$ & & $\downarrow 18 \%$ Tchl & & $\begin{array}{l}\uparrow 274 \% \text { SOD } \\
\uparrow 212 \% \text { POD } \\
\uparrow 204 \% \text { CAT }\end{array}$ & & $\uparrow 41 \%$ & $\begin{array}{l}\text { In shoot: } \downarrow 23 \% \mathrm{Na}^{+}, \\
\uparrow 7 \% \mathrm{~K}^{+}, \uparrow 5 \% \mathrm{Mg}^{2+}, \\
\uparrow 0 \% \mathrm{Ca}^{2+}, \mathrm{Na}^{+} / \mathrm{K}^{+} \text {ratio } \sim 0.67\end{array}$ & \\
\hline
\end{tabular}


Table 1. Cont.

\begin{tabular}{|c|c|c|c|c|c|c|c|c|c|c|}
\hline PGPR & Treatments & GP & Hormones & PhoPs & MDA & AEs & NEAs & Pro & Ion Content & Sources \\
\hline & $\begin{array}{l}10 \text { ppm Boron + } \\
\text { Inoculated }\end{array}$ & $\uparrow 18 \%$ SFW & & $\uparrow 59 \%$ Tchl & & $\begin{array}{l}\uparrow 400 \% \text { SOD } \\
\uparrow 272 \% \text { POD } \\
\uparrow 254 \% \text { CAT }\end{array}$ & & $\uparrow 74 \%$ & $\begin{array}{l}\text { In shoot: } \downarrow 31 \% \mathrm{Na}^{+}, \uparrow 61 \% \mathrm{~K}^{+}, \uparrow \\
67 \% \mathrm{Mg}^{2+}, \uparrow 18 \% \mathrm{Ca}^{2+}, \mathrm{Na}^{+} / \mathrm{K}^{+} \\
\text {ratio } \sim 0.4\end{array}$ & \\
\hline & $\begin{array}{l}150 \mathrm{mM} \mathrm{NaCl}+ \\
\text { Uninoculated }\end{array}$ & $\downarrow 10 \%$ SFW & & $\downarrow 9 \%$ Tchl & & $\begin{array}{l}\uparrow 248 \% \text { SOD } \\
\uparrow 168 \% \text { POD } \\
\uparrow 204 \% \text { CAT }\end{array}$ & & $\uparrow 56 \%$ & $\begin{array}{l}\text { In shoot: } \uparrow 458 \% \mathrm{Na}^{+}, \\
\downarrow 50 \% \mathrm{~K}^{+}, \downarrow 38 \% \mathrm{Mg}^{2+}, \\
\downarrow 76 \% \mathrm{Ca}^{2+}, \\
\mathrm{Na}^{+} / \mathrm{K}^{+} \text {ratio } \sim 10.4\end{array}$ & \\
\hline & $\begin{array}{l}150 \mathrm{mM} \mathrm{NaCl}+ \\
\text { B. pumilus }\end{array}$ & $\uparrow 11 \%$ SFW & & $\uparrow 86 \%$ Tchl & & $\begin{array}{l}\uparrow 348 \% \text { SOD } \\
\uparrow 220 \% \text { POD } \\
\uparrow 273 \% \text { CAT }\end{array}$ & & $\uparrow 83 \%$ & $\begin{array}{l}\text { In shoot: } \uparrow 185 \% \mathrm{Na}^{+}, \\
\uparrow 24 \% \mathrm{Mg}^{2+}, \downarrow 7 \% \mathrm{~K}^{+}, \\
\downarrow 18 \% \mathrm{Ca}^{2+}, \mathrm{Na}^{+} / \mathrm{K}^{+} \text {ratio } 3\end{array}$ & \\
\hline & $\begin{array}{l}10 \mathrm{ppm} \text { Boron + } \\
150 \mathrm{mM} \mathrm{NaCl}+ \\
\text { Uninoculated }\end{array}$ & $\downarrow 10 \% \mathrm{SFW}$ & & $\downarrow 23 \%$ Tchl & & $\begin{array}{l}\uparrow 300 \% \text { SOD } \\
\uparrow 388 \% \text { POD } \\
\uparrow 377 \% \text { CAT }\end{array}$ & & $\uparrow 146 \%$ & $\begin{array}{l}\text { In shoot: } \uparrow 531 \% \mathrm{Na}^{+}, \\
\downarrow 32 \% \mathrm{~K}^{+}, \downarrow 33 \% \mathrm{Mg}^{2+} \\
\downarrow 27 \% \mathrm{Ca}^{2+}, \mathrm{Na}^{+} / \mathrm{K}^{+} \text {ratio } \sim 8.6\end{array}$ & \\
\hline & $\begin{array}{l}10 \text { ppm Boron }+ \\
150 \mathrm{mM} \mathrm{NaCl}+ \\
\text { B. pumilus }\end{array}$ & $\uparrow 3 \%$ SFW & & $\downarrow 5 \%$ Tchl & & $\begin{array}{l}\uparrow 322 \% \text { SOD } \\
\uparrow 316 \% \text { POD } \\
\uparrow 254 \% \text { CAT }\end{array}$ & & $\uparrow 85 \%$ & $\begin{array}{l}\text { In shoot: } \uparrow 115 \% \mathrm{Na}^{+}, \\
\downarrow 11 \% \mathrm{~K}^{+}, \\
\downarrow 5 \% \mathrm{Mg}^{2+}, \downarrow 4 \% \mathrm{Ca}^{2+}, \mathrm{Na}^{+} / \mathrm{K}^{+} \\
\text {ratio } \sim 2.2\end{array}$ & \\
\hline \multirow{6}{*}{$\begin{array}{l}\text { C. gleum SUK. } \\
\text { Wheat plantlets } \\
\text { inoculated with } \\
\text { C. gleum. }\end{array}$} & $\begin{array}{l}0 \mathrm{mM} \mathrm{NaCl}+ \\
\text { Uninoculated }\end{array}$ & & & & & & & & & [99] \\
\hline & $\begin{array}{l}100 \mathrm{mM} \mathrm{NaCl}+ \\
\text { Uninoculated }\end{array}$ & $\begin{array}{l}\downarrow 41 \% \text { SL } \\
\downarrow 46 \% \text { RL } \\
\downarrow 16 \% \text { TPFW }\end{array}$ & \multirow{5}{*}{$\mathrm{N} / \mathrm{A}$} & $\downarrow 36 \%$ Tchl & \multirow{5}{*}{ N/A } & \multirow{5}{*}{$\mathrm{N} / \mathrm{A}$} & $\uparrow 80 \%$ FLA & $\uparrow 31 \%$ & $\begin{array}{l}\text { In shoot: } \uparrow 128 \% \mathrm{Na}^{+}, \\
\downarrow 30 \% \mathrm{~K}^{+}, \mathrm{Na}^{+} / \mathrm{K}^{+} \text {ratio } \sim 0.12\end{array}$ & \\
\hline & $\begin{array}{l}0 \mathrm{mM} \mathrm{NaCl}+ \\
\text { SUK + FLI }\end{array}$ & $\begin{array}{l}\downarrow 13 \% \text { SL } \\
\downarrow 14 \% \text { RL } \\
\downarrow 0 \% \text { TPFW }\end{array}$ & & $\uparrow 18 \%$ Tchl & & & $\uparrow 96 \%$ FLA & $\uparrow 48 \%$ & N/A & \\
\hline & $\begin{array}{l}100 \mathrm{mM} \mathrm{NaCl}+ \\
\text { SUK + FLI }\end{array}$ & $\begin{array}{l}\downarrow 9 \% \text { SL } \\
\downarrow 9 \% \text { RL } \\
\uparrow 19 \% \text { TPFW }\end{array}$ & & $\uparrow 5 \%$ Tchl & & & $\uparrow 147 \%$ FLA & $\uparrow 63 \%$ & $\begin{array}{l}\text { In shoot: } \uparrow 61 \% \mathrm{Na}^{+}, \\
\downarrow 19 \% \mathrm{~K}^{+}, \mathrm{Na}^{+} / \mathrm{K}^{+} \text {ratio } \sim 0.08\end{array}$ & \\
\hline & $\underset{\text { SUK }}{0 \mathrm{mM} \mathrm{NaCl}}+$ & $\begin{array}{l}\downarrow 16 \% \text { SL } \\
\downarrow 36 \% \text { RL } \\
\downarrow 13 \% \text { TPFW }\end{array}$ & & $\downarrow 11 \%$ Tchl & & & $\uparrow 57 \%$ FLA & $\uparrow 25 \%$ & N/A & \\
\hline & $\underset{\text { SUK }}{100 \underset{\mathrm{mM} \mathrm{NaCl}}{+}}$ & $\begin{array}{l}\downarrow 19 \% \text { SL, } \\
\downarrow 9 \% \text { RL, } \downarrow 6 \% \\
\text { TPFW }\end{array}$ & & $\downarrow 23 \%$ Tchl & & & $\uparrow 84 \%$ FLA & $\uparrow 47 \%$ & $\begin{array}{l}\text { In shoot: } \uparrow 67 \% \mathrm{Na}^{+}, \\
\downarrow 19 \% \mathrm{~K}^{+}, \mathrm{Na}^{+} / \mathrm{K}^{+} \text {ratio } 0.08\end{array}$ & \\
\hline
\end{tabular}


Table 1. Cont.

\begin{tabular}{|c|c|c|c|c|c|c|c|c|c|c|}
\hline PGPR & Treatments & GP & Hormones & PhoPs & MDA & AEs & NEAs & Pro & Ion Content & Sources \\
\hline \multirow{4}{*}{$\begin{array}{l}\text { B. aquimaris DY-3. } \\
\text { Three-day-old maize } \\
\text { seedlings inoculated } \\
\text { with DY-3 }\end{array}$} & Control & & & & & & & & & [42] \\
\hline & $\begin{array}{c}1 \%(w / v) \mathrm{NaCl}+ \\
\text { Uninoculated }\end{array}$ & $\downarrow 34 \%$ TPDW & \multirow{3}{*}{ N/A } & $\downarrow 13 \%$ Tchl & $\uparrow 39 \%$ & $\begin{array}{l}\uparrow 21 \% \text { SOD } \\
\uparrow 16 \% \text { POD } \\
\uparrow 18 \% \text { CAT } \\
\uparrow 23 \% \text { APX }\end{array}$ & $\uparrow 22 \%$ PHE & $\uparrow 36 \%$ & \multirow{3}{*}{$\mathrm{N} / \mathrm{A}$} & \\
\hline & $\begin{array}{l}0 \% \mathrm{NaCl}+ \\
\text { DY-3 }\end{array}$ & $\uparrow 12 \%$ TPDW & & $\uparrow 5 \%$ Tchl & $\downarrow 8 \%$ & $\begin{array}{l}\uparrow 13 \% \text { SOD } \\
\uparrow 9 \% \text { CAT } \\
\uparrow 9 \% \text { APX } \\
\downarrow 12 \% \text { POD }\end{array}$ & $\uparrow 11 \%$ PHE & $\uparrow 24 \%$ & & \\
\hline & $\begin{array}{c}1 \%(w / v) \mathrm{NaCl}+ \\
\text { DY-3 }\end{array}$ & $\downarrow 13 \%$ TPDW & & $\downarrow 9 \%$ Tchl & $\uparrow 26 \%$ & $\begin{array}{l}\uparrow 53 \% \text { SOD } \\
\uparrow 42 \% \text { CAT } \\
\uparrow 65 \% \text { APX } \\
\downarrow 2 \% \text { POD }\end{array}$ & $\uparrow 67 \%$ PHE & $\uparrow 77 \%$ & & \\
\hline \multirow{4}{*}{$\begin{array}{l}\text { Klebsiella IG3. } \\
\text { Oat seedlings } \\
\text { inoculated with IG3 }\end{array}$} & Control & & & & & & & & & [44] \\
\hline & $\begin{array}{c}100 \mathrm{mM} \mathrm{NaCl}+ \\
\text { Uninoculated }\end{array}$ & $\begin{array}{l}\downarrow 22 \% \text { SL } \\
\downarrow 31 \% \text { SFW } \\
\downarrow 29 \% \text { RFW } \\
\downarrow 18 \% \text { RL }\end{array}$ & $\downarrow 6 \%$ IAA & $\downarrow 22 \%$ Tchl & $\begin{array}{l}\text { In shoot: } \\
\uparrow 135 \% \\
\text { In root: } \\
\uparrow 231 \%\end{array}$ & $\begin{array}{l}\uparrow 353 \% \text { SOD } \\
\uparrow 540 \% \text { POD }\end{array}$ & \multirow{3}{*}{ N/A } & $\uparrow 230 \%$ & \multirow{3}{*}{ N/A } & \\
\hline & $\begin{array}{l}0 \mathrm{mM} \mathrm{NaCl}+ \\
\text { IG3 }\end{array}$ & $\begin{array}{l}\uparrow 3 \% \mathrm{SL} \\
\uparrow 3 \% \mathrm{SFW} \\
\uparrow 1 \% \mathrm{RFW} \\
\uparrow 13 \% \mathrm{RL}\end{array}$ & $\uparrow 41 \%$ IAA & $\uparrow 4 \%$ Tchl & $\begin{array}{l}\text { In shoot: } \\
\uparrow 3 \% \\
\text { In root: } \\
\uparrow 18 \%\end{array}$ & $\begin{array}{l}\uparrow 0 \% \text { SOD } \\
\uparrow 2 \% \text { POD }\end{array}$ & & $\uparrow 42 \%$ & & \\
\hline & $\underset{\text { IG3 }}{100 \mathrm{mM} \mathrm{NaCl}+}$ & $\begin{array}{l}\downarrow 10 \% \text { SL, } \\
\downarrow 18 \% \text { SFW } \\
\downarrow 16 \% \text { RFW } \\
\downarrow 2 \% \text { RL }\end{array}$ & $\uparrow 67 \%$ IAA & $\downarrow 13 \%$ Tchl & $\begin{array}{l}\text { In shoot: } \\
\uparrow 27 \% \\
\text { In root: } \\
\uparrow 45 \%\end{array}$ & $\begin{array}{l}\uparrow 96 \% \text { SOD } \\
\uparrow 286 \% \text { POD }\end{array}$ & & $\uparrow 155 \%$ & & \\
\hline \multirow{3}{*}{$\begin{array}{l}\text { P. yonginensis DCY84. } \\
\text { Root seedlings of } \\
\text { ginseng inoculated } \\
\text { with P. yonginensis } \\
\text { DCY84 }\end{array}$} & \multicolumn{9}{|c|}{ Short period of stress ( 3 days of $300 \mathrm{mM} \mathrm{NaCl}$ exposure) } & \multirow[t]{3}{*}{ [50] } \\
\hline & Control & & & & & & & & $\begin{array}{l}\text { In shoot: } \mathrm{Na}^{+} / \mathrm{K}^{+} \text {ratio } \sim 13 \\
\text { In root: } \mathrm{Na}^{+} / \mathrm{K}^{+} \text {ratio } \sim 11\end{array}$ & \\
\hline & $\underset{\text { DCY } 84}{0 \mathrm{mM} \mathrm{NaCl}}+$ & $\begin{array}{l}\uparrow 15 \% \text { SFW } \\
\uparrow 5 \% \text { RFW }\end{array}$ & N/A & $\begin{array}{l}\uparrow 3 \% \text { Chl a } \\
\uparrow 2 \% \text { Chl b } \\
\uparrow 2 \% \text { Car }\end{array}$ & Unchange & $\begin{array}{r}\uparrow 62 \% \text { APX } \\
d \uparrow 40 \% \text { POD } \\
\uparrow 114 \% \text { CAT }\end{array}$ & N/A & $\uparrow 253 \%$ & $\begin{array}{l}\text { In shoot: } \mathrm{Na}^{+} / \mathrm{K}^{+} \text {ratio } \sim 15 \\
\text { In root: } \mathrm{Na}^{+} / \mathrm{K}^{+} \text {ratio } \sim 11\end{array}$ & \\
\hline
\end{tabular}


Table 1. Cont.

\begin{tabular}{|c|c|c|c|c|c|c|c|c|c|c|}
\hline PGPR & Treatments & GP & Hormones & PhoPs & MDA & AEs & NEAs & Pro & Ion Content & Sources \\
\hline & $\begin{array}{l}300 \mathrm{mM} \mathrm{NaCl}+ \\
\text { Uninoculated }\end{array}$ & $\begin{array}{l}\downarrow 13 \% \text { SFW } \\
\downarrow 9 \% \text { RFW }\end{array}$ & & $\begin{array}{l}\downarrow 15 \% \text { Chl a } \\
\downarrow 13 \% \text { Chl b } \\
\downarrow 16 \% \text { Car }\end{array}$ & $\uparrow 29 \%$ & $\begin{array}{l}\uparrow 55 \% \mathrm{POD} \\
\uparrow 0 \% \mathrm{APX} \\
\downarrow 14 \% \mathrm{CAT}\end{array}$ & & $\uparrow 20 \%$ & $\begin{array}{l}\text { In shoot: } \mathrm{Na}^{+} / \mathrm{K}^{+} \text {ratio } \sim 6.4 \\
\text { In root: } \mathrm{Na}^{+} / \mathrm{K}^{+} \text {ratio } \sim 7\end{array}$ & \\
\hline & $\begin{array}{l}300 \mathrm{mM} \mathrm{NaCl}+ \\
\text { DCY84 }\end{array}$ & $\begin{array}{l}\uparrow 12 \% \text { SFW } \\
\uparrow 0 \% \text { RFW }\end{array}$ & & $\begin{array}{l}\uparrow 3 \% \mathrm{Chl} \mathrm{a} \\
\downarrow 2 \% \mathrm{Chl} \text { b } \\
\downarrow 9 \% \mathrm{Car}\end{array}$ & $\uparrow 21 \%$ & $\begin{array}{l}\uparrow 54 \% \text { APX } \\
\uparrow 80 \% \text { POD } \\
\uparrow 114 \% \text { CAT }\end{array}$ & & $\uparrow 233 \%$ & $\begin{array}{l}\text { In shoot: } \mathrm{Na}^{+} / \mathrm{K}^{+} \text {ratio } \sim 8.2 \\
\text { In root: } \mathrm{Na}^{+} / \mathrm{K}^{+} \text {ratio } \sim 9.8\end{array}$ & \\
\hline & \multicolumn{9}{|c|}{ Long period of stress (12 days of $300 \mathrm{mM} \mathrm{NaCl}$ exposure) } & \\
\hline & Control & & & & & & & & $\begin{array}{l}\text { In shoot: } \mathrm{Na}^{+} / \mathrm{K}^{+} \text {ratio } \sim 7.2 ; \text { In } \\
\text { root: } \mathrm{Na}^{+} / \mathrm{K}^{+} \text {ratio } \sim 9.9\end{array}$ & \\
\hline & $\underset{\text { DCY } 84}{0 \mathrm{mM} \mathrm{NaCl}}+$ & $\begin{array}{l}\uparrow 17 \% \text { SFW } \\
\uparrow 1 \% \text { RFW }\end{array}$ & \multirow{3}{*}{ N/A } & $\begin{array}{l}\uparrow 3 \% \mathrm{Chl} \mathrm{a} \\
\uparrow 2 \% \mathrm{Chl} b \\
\downarrow 2 \% \mathrm{Car}\end{array}$ & Unchange & $\begin{array}{l}\uparrow 45 \% \text { APX } \\
d \uparrow 100 \% \text { POD } \\
\uparrow 143 \% \text { CAT }\end{array}$ & \multirow{3}{*}{$\mathrm{N} / \mathrm{A}$} & $\uparrow 300 \%$ & $\begin{array}{l}\text { In shoot: } \mathrm{Na}^{+} / \mathrm{K}^{+} \text {ratio } \sim 7.8 \\
\text { In root: } \mathrm{Na}^{+} / \mathrm{K}^{+} \text {ratio } \sim 11\end{array}$ & \\
\hline & $\begin{array}{l}300 \mathrm{mM} \mathrm{NaCl}+ \\
\text { Uninoculated }\end{array}$ & $\begin{array}{l}\downarrow 18 \% \text { SFW } \\
\downarrow 22 \% \text { RFW }\end{array}$ & & $\begin{array}{l}\downarrow 53 \% \text { Chl a } \\
\downarrow 66 \% \text { Chl b } \\
\downarrow 57 \% \text { Car }\end{array}$ & $\uparrow 36 \%$ & $\begin{array}{l}\downarrow 31 \% \text { APX } \\
\downarrow 33 \% \text { POD } \\
\downarrow 71 \% \text { CAT }\end{array}$ & & $\uparrow 13 \%$ & $\begin{array}{l}\text { In shoot: } \mathrm{Na}^{+} / \mathrm{K}^{+} \text {ratio } \sim 4.4, \\
\text { In root: } \mathrm{Na}^{+} / \mathrm{K}^{+} \text {ratio } \sim 3.8\end{array}$ & \\
\hline & $\begin{array}{c}300 \mathrm{mM} \mathrm{NaCl}+ \\
\text { DCY84 }\end{array}$ & $\begin{array}{l}\uparrow 12 \% \text { SFW } \\
\downarrow 3 \% \text { RFW }\end{array}$ & & $\begin{array}{l}\downarrow 3 \% \mathrm{Chl} \mathrm{a} \\
\downarrow 11 \% \mathrm{Chl} \mathrm{b} \\
\downarrow 7 \% \mathrm{Car}\end{array}$ & $\uparrow 14 \%$ & $\begin{array}{l}\uparrow 90 \% \text { APX } \\
\uparrow 317 \% \text { POD } \\
\uparrow 343 \% \text { CAT }\end{array}$ & & $\uparrow 287 \%$ & $\begin{array}{l}\text { In shoot: } \mathrm{Na}^{+} / \mathrm{K}^{+} \text {ratio } \sim 6.5, \\
\text { In root: } \mathrm{Na}^{+} / \mathrm{K}^{+} \text {ratio } \sim 7.7\end{array}$ & \\
\hline \multirow{3}{*}{$\begin{array}{l}\text { B. megaterium A12 } \\
\text { (BMA12). } \\
\text { Ten-day-old tomato } \\
\text { seedlings inoculated } \\
\text { with BMA12 }\end{array}$} & Control & & & & & & & & & [45] \\
\hline & $\begin{array}{l}200 \mathrm{mM} \mathrm{NaCl}+ \\
\text { Uninoculated }\end{array}$ & $\begin{array}{l}\downarrow 37 \% \text { PH } \\
\downarrow 50 \% \text { RL } \\
\downarrow 59 \% \text { TPFW } \\
\downarrow 54 \% \text { TPDW } \\
\downarrow 35 \% \text { TLA }\end{array}$ & $\begin{array}{l}\downarrow 32 \% \text { IAA } \\
\downarrow 43 \% \text { GA4 } \\
\uparrow 100 \% \\
\mathrm{C}_{2} \mathrm{H}_{4} \uparrow 82 \% \\
\text { ABA }\end{array}$ & $\begin{array}{l}\downarrow 32 \% \text { Chl a } \\
\downarrow 40 \% \text { Chl b } \\
\downarrow 41 \% \text { TChl } \\
\downarrow 49 \% \text { Car }\end{array}$ & \multirow{2}{*}{ N/A } & $\begin{array}{l}\uparrow 24 \% \text { SOD } \\
\uparrow 27 \% \text { CAT } \\
\downarrow 24 \% \text { APX } \\
\downarrow 24 \% \text { POD } \\
\downarrow 57 \% \text { PPO }\end{array}$ & $\begin{array}{l}\uparrow 74 \% \mathrm{GSH} \\
\uparrow 228 \% \mathrm{ASC}\end{array}$ & \multirow[b]{2}{*}{ N/A } & \multirow[b]{2}{*}{$\mathrm{N} / \mathrm{A}$} & \\
\hline & $\underset{\text { BMA12 }}{0 \mathrm{mM} \mathrm{NaCl}}+$ & $\begin{array}{l}\uparrow 23 \% \text { PH } \\
\uparrow 37 \% \text { RL } \\
\uparrow 28 \% \text { TPFW } \\
\uparrow 40 \% \text { TPDW } \\
\uparrow 25 \% \text { TLA }\end{array}$ & $\begin{array}{l}\uparrow 53 \% \text { IAA, } \\
\uparrow 170 \% \text { GA4 } \\
\downarrow 16 \% \mathrm{C}_{2} \mathrm{H}_{4} \\
\downarrow 14 \% \mathrm{ABA}\end{array}$ & $\begin{array}{l}\uparrow 53 \% \text { Chl a } \\
\uparrow 14 \% \text { Chl b } \\
\uparrow 41 \% \text { TChl } \\
\uparrow 35 \% \text { Car }\end{array}$ & & $\begin{array}{l}\uparrow 86 \% \text { SOD } \\
\uparrow 54 \% \text { CAT } \\
\uparrow 34 \% \text { APX } \\
\uparrow 37 \% \text { POD } \\
\uparrow 55 \% \text { PPO }\end{array}$ & $\begin{array}{l}\uparrow 17 \% \text { GSH } \\
\uparrow 5 \% \text { ASC }\end{array}$ & & & \\
\hline
\end{tabular}


Table 1. Cont.

\begin{tabular}{|c|c|c|c|c|c|c|c|c|c|c|}
\hline PGPR & Treatments & GP & Hormones & PhoPs & MDA & AEs & NEAs & Pro & Ion Content & Sources \\
\hline & $\begin{array}{c}2000 \mathrm{mM} \mathrm{NaCl}+ \\
\text { BMA12 }\end{array}$ & $\begin{array}{l}\downarrow 13 \% \text { PH } \\
\downarrow 28 \% \text { RL } \\
\downarrow 33 \% \text { TPFW } \\
\downarrow 35 \% \text { TPDW } \\
\downarrow 21 \% \text { TLA }\end{array}$ & $\begin{array}{l}\uparrow 0 \% \text { IAA } \\
\uparrow 11 \% \mathrm{C}_{2} \mathrm{H}_{4} \\
\uparrow 186 \% \text { ABA, } \\
\uparrow 86 \% \text { GA4 }\end{array}$ & $\begin{array}{l}\uparrow 5 \% \text { Chl a } \\
\downarrow 17 \% \text { Chl b } \\
\downarrow 4 \% \text { TChl } \\
\downarrow 24 \% \text { Car }\end{array}$ & & $\begin{array}{l}\uparrow 213 \% \text { SOD } \\
\uparrow 91 \% \text { CAT } \\
\uparrow 78 \% \text { APX } \\
\uparrow 18 \% \text { POD } \\
\downarrow 10 \% \text { PPO }\end{array}$ & $\begin{array}{l}\uparrow 250 \% \text { GSH } \\
\uparrow 100 \% \text { ASC }\end{array}$ & & & \\
\hline \multirow{7}{*}{$\begin{array}{l}\text { Pseudomonas } \\
\text { (wild-type UW4 and } \\
\text { mutant strains). } \\
\text { Seven-day-old } \\
\text { tomato plants } \\
\text { inoculated with } \\
\text { UW4. }\end{array}$} & Control & & & & & & & & & [46] \\
\hline & $\begin{array}{c}200 \mathrm{mM} \mathrm{NaCl}+ \\
\text { Uninoculated }\end{array}$ & $\begin{array}{l}\downarrow 56 \% \text { RL } \\
\downarrow 37 \% \text { SL } \\
\downarrow 37 \% \text { TPDW }\end{array}$ & \multirow{6}{*}{ N/A } & $\downarrow 42 \%$ Tchl & & & & \multirow{6}{*}{ N/A } & & \\
\hline & $\begin{array}{c}200 \mathrm{mM} \mathrm{NaCl}+ \\
\text { WT UW4 }\end{array}$ & $\begin{array}{l}\uparrow 16 \% \text { RL } \\
\uparrow 3 \% \text { SL } \\
\uparrow 25 \% \text { TPDW }\end{array}$ & & $\uparrow 31 \%$ Tchl & & & & & & \\
\hline & $\begin{array}{c}200 \mathrm{mM} \mathrm{NaCl}+ \\
\text { acdS-mutant }\end{array}$ & $\begin{array}{l}\downarrow 33 \% \text { RL } \\
\downarrow \text { } 9 \% \text { SL } \\
\downarrow 17 \% \text { TPDW }\end{array}$ & & $\downarrow 25 \%$ Tchl & & & & & & \\
\hline & $\begin{array}{c}200 \mathrm{mM} \mathrm{NaCl}+ \\
\text { treS- mutant }\end{array}$ & $\begin{array}{l}\downarrow 39 \% \text { RL } \\
\downarrow 31 \% \text { SL } \\
\downarrow 8 \% \text { TPDW }\end{array}$ & & $\downarrow 13 \%$ Tchl & & & & & & \\
\hline & $\begin{array}{c}200 \mathrm{mM} \mathrm{NaCl}+ \\
\text { acdS-/treS- } \\
\text { double mutant }\end{array}$ & $\begin{array}{l}\downarrow 58 \% \text { RL } \\
\downarrow 37 \% \text { SL } \\
\downarrow 35 \% \text { TPDW }\end{array}$ & & $\downarrow 56 \%$ Tchl & & & & & & \\
\hline & $\begin{array}{l}200 \mathrm{mM} \mathrm{NaCl}+ \\
\text { OxtreS }\end{array}$ & $\begin{array}{l}\uparrow 45 \% \mathrm{RL} \\
\uparrow 3 \% \mathrm{SL} \\
\uparrow 54 \% \mathrm{TPDW}\end{array}$ & & $\uparrow 61 \%$ Tchl & & & & & & \\
\hline \multirow{2}{*}{$\begin{array}{l}\text { S. maltophilia BJ01. } \\
\text { Seven-day-old } \\
\text { peanut seedlings } \\
\text { inoculated with BJ01 }\end{array}$} & Control & & & & & & & & & [48] \\
\hline & $\begin{array}{c}0 \mathrm{mM} \mathrm{NaCl}+ \\
\text { BJ01 }\end{array}$ & $\begin{array}{l}\uparrow 4 \% \mathrm{SL}, \\
\uparrow 11 \% \mathrm{TPFW}, \\
\downarrow 15 \% \mathrm{RL}\end{array}$ & $\uparrow 19 \%$ Aux & $\begin{array}{l}\uparrow 11 \% \text { Chla, } \\
\uparrow 0 \% \text { Chl b, } \\
\uparrow 17 \% \text { Tchl }\end{array}$ & $\downarrow 26 \%$ & $\mathrm{~N} / \mathrm{A}$ & & $\downarrow 32 \%$ & N/A & \\
\hline
\end{tabular}


Table 1. Cont.

\begin{tabular}{|c|c|c|c|c|c|c|c|c|c|c|}
\hline PGPR & Treatments & GP & Hormones & PhoPs & MDA & AEs & NEAs & Pro & Ion Content & Sources \\
\hline & $\begin{array}{l}100 \mathrm{mM} \mathrm{NaCl}+ \\
\text { Uninoculated }\end{array}$ & $\begin{array}{l}\uparrow 9 \% \text { RL, } \\
\downarrow 45 \% \text { TPFW, } \\
\downarrow 39 \% \text { SL }\end{array}$ & $\uparrow 16 \%$ Aux & $\begin{array}{l}\downarrow 56 \% \text { Chl a, } \\
\downarrow 42 \% \text { Chl b, } \\
\downarrow 50 \% \text { Tchl }\end{array}$ & $\uparrow 47 \%$ & & & $\uparrow 1355 \%$ & & \\
\hline & $\begin{array}{c}100 \mathrm{mM} \mathrm{NaCl}+ \\
\text { BJ01 }\end{array}$ & $\begin{array}{l}\downarrow 26 \% \text { SL, } \\
\downarrow 3 \% \text { RL, } \\
\downarrow 26 \% \text { TPFW }\end{array}$ & $\uparrow 29 \%$ Aux & $\begin{array}{l}\downarrow 11 \% \text { Chl a } \\
\downarrow 34 \% \text { Chl b } \\
\downarrow 23 \% \text { Tchl }\end{array}$ & $\uparrow 16 \%$ & & & $\uparrow 1173 \%$ & & \\
\hline
\end{tabular}

Note: All calculations in the Table 1 represent the comparisons between the treated plants and the control plants (non-stress conditions and un-inoculation). The up arrowhead ( $\uparrow$ ) indicates an increase in a tested parameter as compared to the control. The down arrowhead $(\downarrow)$ displays a reduction in a tested parameter relative to the control. Abbreviation in the Table 1 : ABA, Abscisic acid; A. calcoaceticus, Acinetobacter calcoaceticus; A. aneurinilyticus, Aneurinibacillus aneurinilyticus; AEs, antioxidant enzymes; APX, Ascorbate peroxidase; ASC, Ascorbate; Aux, Auxin; B. aquimaris, Bacillus aquimaris; B. brevis, Bacillus brevis; B. cepacia, Burkholdera cepacia; B. megaterium, Bacillus megaterium; B. pumilus, Bacillus pumilus; $\mathrm{C}_{2} \mathrm{H}_{4}$, ethylene; Car, Carotenoids; CAT, Catalase; C. gleum, Chryseobacterium gleum; Chl, Chlorophyll; E. aerogenes, Enterobacter aerogenes; FLA, Flavonoids; FLI, Feather lysate inoculum; GA4, Gibberellins 4; GSH, Glutathione; GP, Growth parameter; IAA, Indole-3-acetic acid; JA, Jasmonates; MDA, Malondialdehyde; N/A, Not available; NEAs, Non-enzymatic antioxidants; P. fluorescence, Pseudomonas fluorescence; PH, Plant height; PHE, Phenols; PhoPs, Photosynthetic pigments; POD, Peroxidase; PPs, Polyphenols; Pro, Proline; P. putida, Pseudomonas putida; PPO, Polyphenol oxidase; Pro, Proline; P. yonginensis, Paenibacillus yonginensis; RDW, Root dry weight; RFW, Root fresh weight; RL, Root length; SA, Salicylic acid; SDW, Shoot dry weight; SFW, Shoot fresh weight; SL, Shoot length; S. maltophilia, Stenotrophomonas maltophilia; SOD, Superoxide dismutase; Tchl, Total chlorophyll; TLA, Total leaves area per plant; TPDW, Total plant dry weight; TPFW, Total plant fresh weight; TPP, Total polyphenol; X. autotrophicus, Xanthobacter autotrophicus; Y, Yield. 
In the study of Sarkar et al. [57], the inoculation of rice seeds with Enterobacter sp. strain P23 promoted higher germination percentage (GP) (76\% \pm 7.03 vs. $48 \% \pm 4.78)$, and higher seedling vigor index (SVI) $(881.6 \pm 67 \mathrm{vs}$. $57.5 \pm 12.6)$ as compared to the non-inoculated seeds. Under salt conditions, the Pro peaked its highest level, the SOD, CAT, POD, PPO, and MDA exhibited their highest contents in uninoculated rice seedlings. However, the activities of these enzymes in P23-inoculated seedlings were significantly reduced relative to those in the non-inoculated seedlings. The productions of ethylene in non-inoculated seedlings and P23 AcdS mutant-inoculated seedlings were comparable, consistent with the study of Cheng et al. [30], while ethylene in the WT P23 strain-treated plants was lower, indicating that the WT P23 succeeded in decreasing stress ethylene production. Under $250 \mathrm{mM} \mathrm{NaCl}$ treatment, the SFW and SDW of P. putida UW 4-inoculated canola plants (Brassica napus L.) were 1.7-fold higher than those of untreated plants [30]. However, the P. putida ACC deaminase (AcdS) minus mutant-inoculated canola plants did not show significant difference in SFW and SDW relative to the untreated plants, indicating the critical role of a functional ACC deaminase enzyme in plant growth under salinity stress. The proteins involved in photosynthesis in the WT P. putida UW4 plants were downregulated; however, to a lesser extent as compared to that in the uninoculated plants or in the P. putida AcdS plants, resulting in the higher chlorophyll contents relative to the uninoculated plants. Surprisingly, both AcdS and WT P. putida plants could accumulate large amount of $\mathrm{NaCl}$ in their shoots with 3.7-7-fold higher than that in the uninoculated plants, respectively while being able to maintain their normal growth. This could be partly explained by the increase cell permeability caused by IAA that was produced by the WT P. putida and the AcdS mutant. This finding is intriguing and controversial since numerous other studies recognized the decreased $\mathrm{Na}^{+}$uptake in PGPR-bacterized plants $[40,42,99,111]$. In their another study, Sarkar et al. (2018) [49] primed the rice seeds (Oryza sativa cv. Ratna) with Enterobacter sp. P23 and achieved greater GRA (76\% vs. 48\%), as well as SVI (881.6 vs. 57.6 ) relative to the unprimed seeds. Subsequently, the growth and development of the primed seedlings were better than the unprimed control, representing via greater SFW, RFW, SDW, RDW, SL, RL, amylase, protease, Aux, and Chl values $[49,57]$. In the study of Zhu et al. (2020) [107], the treatment with $130 \mathrm{mM} \mathrm{NaCl}$ severely affected the GRA of the non-primed alfalfa seeds (Medicago sativa L.) in comparison with the primed seeds. Specifically, the gemination rate of the non-primed seeds reduced to $29 \%$ versus $32 \%$ of B. megaterium NRCB001-primed seeds, $42 \%$ of B. subtilis NRCB002, and $40 \%$ of B. subtilis NRCB003. Also in Zhu et al. [107], the vegetative parameters such as PH, RL, NL, TLA, and TPDW of primed seedlings were always higher than those of unprimed seedlings and the MDA content in their leaves were lower, suggesting a less injured cellular membrane in the primed alfalfa grass.

Regarding the synergy between different PGPR and/or between the microbes and chemicals, the synergistic effects of a consortium (A. aneurinilyticus + Paenibacillus sp.) were observed via the maximum physio-morphology parameters of primed French bean seedlings (Phaseolus vulgaris) in comparison to uninoculated- or individual A. aneurinilyticus and Paenibacillus-primed seedlings [88]. Two VOCs, namely, 4-nitroguaiacol and quinoline derived from Pseudomonas simiae exhibited their ability to induce soybean seed germination under $100 \mathrm{mM} \mathrm{NaCl}$ treatment [147]. Furthermore, the combined treatment of sodium nitroprusside (SNP) and P. simiae resulted in the higher biomass, the lower MDA content and EL in the treated soybean plants than other treatment plants [147]. Mel exhibits pleiotropic biological activities such as growth regulation [148] and antioxidative property [149] and has been widely used as a promising tool for mitigating salt stress in plants. Abd El-Ghany and Attia (2020) [124] found that the combination of Mel and peat-based inoculants (Rhizobium leguminosarum, a N fixing bacterium, and Azotobacter chroococcum, an EPS-producing bacterium) synergistically enhanced salt stress tolerance in faba bean plants (Vicia faba) as compared to Mel- or inoculants-treated seeds alone. Specifically, in the combined treatment (100 $\mu \mathrm{M} \mathrm{Mel}+$ inoculants), the content of $\mathrm{Chl} \mathrm{a}, \mathrm{Chl} \mathrm{b}$, Car, and Pro reached the highest, suggesting the synergistic effects of Mel and beneficial PGPR in improving plant growth 
and other physiological aspects in salt stress conditions. The combination of Mel and bacterial inoculants, in contrast, helped to boost the faba bean plant growth, to increase PhoPs, Pro, N-P-K uptake, and to reduce the $\mathrm{Na}^{+} / \mathrm{K}^{+}$ratio.

In conclusion, seed biopriming using PGPR enhances the GRA and SV index in the primed seeds as compared to the unprimed seeds under saline conditions, thereby supporting plants a vigorous growth and a better salinity tolerance during their whole life [150]. The key findings in recent seed biopriming studies were presented in Table 2.

3.2. The Increase in AEs and/or Osmoregulators in PGPR-Inoculated Plants and PGPR-Primed Seedlings under Salt Stress

The increased activities of AEs and/or the elevated accumulations of osmoregulators in PGPB-inoculated plants were reported by Li and Jiang [42], Akram et al. [45], Vaishnav et al. [47], Khalid et al. [58], Kim et al. [96], Habib et al. [97], Kang et al. [104], Zhu et al. [107], Halo et al. [151], Bharti et al. [152], El-Esawi et al. [153], Vimal et al. [154], El-Nahrawy and Yassin [155], Sun et al. [156]. For instance, the activity of ROS-scavenging enzymes SOD, CAT of Enterobacter-treated okra plants was the highest amongst all treatments, in parallel with their highest vegetative parameters SFW, SDW, RFW, and RDW [97]. Likewise, APX activity in Enterobacter-inoculated tomato plants was 20\% higher and 2,2-diphenyl-1-picryl-hydrazyl-hydrate (DPPH) assay showed 24\% increase in scavenging capacity in the inoculated plants relative to the control plants [96]. In the study of Abd_Allah et al. [35], the activities of POD, CAT, GR, and SOD, and the contents of AsA, GSH and proline were always the highest in the inoculated chickpea plants. The Arabidopsis plants inoculated with Burkholderia phytofirmans PsJN revealed an elevated Pro accumulation in comparison with the control plants [157]. In the Leclercia adcarboxylatatreated tomato plants, Pro, serine (Ser), glycine (Gly), methionine (Met), and threonine (Thr), as well as citric acid (CA) and malic acid (MA) were significantly accumulated [104]. The detailed profiles of AEs were presented in Tables 1 and 2. 
Table 2. The use of PGPR in seed biopriming technique for improving salinity stress tolerance in plants.

\begin{tabular}{|c|c|c|c|c|c|c|c|c|c|}
\hline PGPR & Treatments & GP & PhoPs & AEs & MDA & Ion Content & Pro & Ethylene & Sources \\
\hline \multirow{6}{*}{$\begin{array}{l}\text { S. maltophilia } \\
\text { SBP-9. } \\
\text { Bacterized wheat } \\
\text { seeds with } S \text {. } \\
\text { maltophilia } \\
\text { SBP-9 for } 1 \mathrm{~h} \text {. }\end{array}$} & Control & & & & & & & & \multirow{6}{*}{ [102] } \\
\hline & $\begin{array}{l}0 \mathrm{mM} \mathrm{NaCl}+ \\
\text { SBP-9 }\end{array}$ & $\begin{array}{l}\uparrow 15 \% \mathrm{SL}, \uparrow 10 \% \mathrm{RL}, \uparrow 12 \% \mathrm{SFW}, \\
\uparrow 17 \% \mathrm{SDW}, \uparrow 33 \% \mathrm{RFW}, \\
\uparrow 9 \% \mathrm{RDW}\end{array}$ & $\uparrow 8 \%$ Tchl & $\begin{array}{l}\uparrow 33 \% \text { SOD } \\
\uparrow 20 \% \text { CAT } \\
\uparrow 33 \% \text { POD }\end{array}$ & $\downarrow 27 \%$ & $\begin{array}{l}\text { In shoot: } \downarrow 4 \% \mathrm{Na}^{+}, \uparrow \\
12 \% \mathrm{~K}^{+}, \mathrm{Na}^{+} / \mathrm{K}^{+} \text {ratio } \\
\sim 0.54\end{array}$ & $\downarrow 10 \%$ & \multirow{5}{*}{$\mathrm{N} / \mathrm{A}$} & \\
\hline & $\begin{array}{l}150 \mathrm{mM} \mathrm{NaCl} \\
\text { + Unprimed }\end{array}$ & $\begin{array}{l}\downarrow 11 \% \text { SL, } \downarrow 5 \% \text { RL, } \downarrow 8 \% \text { SFW, } \\
\downarrow 24 \% \text { SDW, } \downarrow 11 \% \text { RFW, } \\
\downarrow 23 \% \text { RDW }\end{array}$ & $\downarrow 15 \%$ Tchl & $\begin{array}{l}\uparrow 58 \% \text { SOD } \\
\uparrow 7 \% \text { CAT } \\
\uparrow 67 \% \text { POD }\end{array}$ & $\uparrow 17 \%$ & $\begin{array}{l}\text { In shoot: } \uparrow 48 \% \mathrm{Na}^{+}, \downarrow \\
17 \% \mathrm{~K}^{+}, \mathrm{Na}^{+} / \mathrm{K}^{+} \text {ratio } \\
\sim 1.12\end{array}$ & $\uparrow 74 \%$ & & \\
\hline & $\begin{array}{l}150 \mathrm{mM} \mathrm{NaCl} \\
+ \text { SBP-9 }\end{array}$ & $\begin{array}{l}\uparrow 4 \% \mathrm{SL}, \uparrow 15 \% \mathrm{RL}, \uparrow 4 \% \mathrm{SFW}, \\
\uparrow 7 \% \mathrm{SDW}, \uparrow 22 \% \mathrm{RFW}, \downarrow 5 \% \mathrm{RDW}\end{array}$ & $\uparrow 5 \%$ Tchl & $\begin{array}{l}\uparrow 133 \% \text { SOD } \\
\uparrow 93 \% \text { CAT } \\
\uparrow 133 \% \text { POD }\end{array}$ & $\downarrow 13 \%$ & $\begin{array}{l}\text { In shoot: } \uparrow 20 \% \mathrm{Na}^{+}, \uparrow \\
3 \% \mathrm{~K}^{+} \mathrm{Na}^{+} / \mathrm{K}^{+} \text {ratio } \\
\sim 0.73\end{array}$ & $\uparrow 23 \%$ & & \\
\hline & $\begin{array}{l}200 \mathrm{mM} \mathrm{NaCl} \\
\text { + Unprimed }\end{array}$ & $\begin{array}{l}\downarrow 37 \% \text { SL }, \downarrow 20 \% \text { RL }, \downarrow 32 \% \text { SFW, } \\
\downarrow 38 \% \text { SDW, } \downarrow 33 \% \text { RFW, } \\
\downarrow 64 \% \text { RDW }\end{array}$ & $\downarrow 59 \%$ Tchl & $\begin{array}{l}\uparrow 217 \% \text { SOD } \\
\uparrow 100 \% \text { CAT } \\
\uparrow 192 \% \text { POD }\end{array}$ & $\uparrow 93 \%$ & $\begin{array}{l}\text { In shoot: } \uparrow 107 \% \mathrm{Na}^{+}, \downarrow \\
25 \% \mathrm{~K}^{+}, \mathrm{Na}^{+} / \mathrm{K}^{+} \text {ratio } \\
\sim 1.73\end{array}$ & $\uparrow 165 \%$ & & \\
\hline & $\begin{array}{l}200 \mathrm{mM} \mathrm{NaCl} \\
+ \text { SBP-9 }\end{array}$ & $\begin{array}{l}\downarrow 11 \% \mathrm{SL}, \downarrow 5 \% \text { RL }, \downarrow 16 \% \mathrm{SFW}, \\
\downarrow 17 \% \mathrm{SDW}, \downarrow 6 \% \text { RFW, } \\
\downarrow 41 \% \mathrm{RDW}\end{array}$ & $\downarrow 39 \%$ Tchl & $\begin{array}{l}\uparrow 350 \% \text { SOD } \\
\uparrow 180 \% \text { CAT } \\
\uparrow 283 \% \text { POD }\end{array}$ & $\uparrow 50 \%$ & $\begin{array}{l}\text { In shoot: } \uparrow 54 \% \mathrm{Na}^{+}, \downarrow \\
3 \% \mathrm{~K}^{+} \mathrm{Na}^{+} / \mathrm{K}^{+} \text {ratio } \sim 1\end{array}$ & $\uparrow 110 \%$ & & \\
\hline \multirow{3}{*}{$\begin{array}{l}\text { Enterobacter P23. } \\
\text { Seeds of Oryza } \\
\text { sativa cv. Ratna } \\
\text { treated with } \\
\text { bacterial } \\
\text { suspension. }\end{array}$} & Control & & & & & & & \multirow{3}{*}{ N/A } & \multirow{3}{*}{ [49] } \\
\hline & $\begin{array}{l}150 \mathrm{mM} \mathrm{NaCl} \\
+ \text { Unprimed }\end{array}$ & $\begin{array}{l}\downarrow 51 \% \text { GP }, \downarrow 97 \% \text { SVI, } \downarrow 45 \% \text { SFW, } \\
\downarrow 58 \% \text { SDW, } \downarrow 33 \% \text { SL, } \\
\downarrow 39 \% \text { RFW, } \downarrow 63 \% \text { RDW, } \downarrow 44 \% \text { RL }\end{array}$ & $\begin{array}{l}\downarrow 54 \% \text { Chl a } \\
\downarrow 80 \% \text { Chl b } \\
\downarrow 56 \% \text { Tchl }\end{array}$ & $\begin{array}{l}\uparrow 120 \% \text { SOD } \\
\uparrow 112 \% \text { CAT } \\
\uparrow 174 \% \text { POD } \\
\uparrow 700 \% \text { PPO }\end{array}$ & $\uparrow 300 \%$ & \multirow[t]{2}{*}{$\mathrm{N} / \mathrm{A}$} & $\uparrow 175 \%$ & & \\
\hline & $\begin{array}{l}150 \mathrm{mM} \mathrm{NaCl} \\
+\mathrm{P} 23\end{array}$ & $\begin{array}{l}\downarrow 22 \% \text { GP, } \downarrow 58 \% \text { SVI }, \downarrow 16 \% \text { SFW, } \\
\downarrow 11 \% \text { SL, } \downarrow 23 \% \text { SDW, } \\
\downarrow 15 \% \text { RFW, } \downarrow 30 \% \text { RDW, } \downarrow 11 \% \text { RL }\end{array}$ & $\begin{array}{l}\downarrow 13 \% \text { Chl a } \\
\downarrow 10 \% \text { Chl b } \\
\downarrow 8 \% \text { Tchl }\end{array}$ & $\begin{array}{l}\uparrow 32 \% \mathrm{SOD} \\
\uparrow 46 \% \mathrm{CAT} \\
\uparrow 70 \% \mathrm{POD} \\
\uparrow 300 \% \mathrm{PPO}\end{array}$ & $\uparrow 195 \%$ & & $\uparrow 75 \%$ & & \\
\hline \multirow{3}{*}{$\begin{array}{l}\text { B. pumilus FAB10. } \\
\text { Wheat seeds cv. } \\
343 \text { treated with } \\
\text { FAB10. }\end{array}$} & Control & & & & & & & & \multirow{3}{*}{ [51] } \\
\hline & $\begin{array}{l}75 \mathrm{mM} \mathrm{NaCl}+ \\
\text { Unprimed }\end{array}$ & $\begin{array}{l}\downarrow 17 \% \text { SL }, \downarrow 35 \% \text { RL, } \downarrow 49 \% \text { SDW, } \\
\downarrow 53 \% \text { RDW, } \downarrow 35 \% \text { SpDW, } \\
\downarrow 21 \% \text { GY, } \downarrow 17 \% \text { GPr }\end{array}$ & & $\begin{array}{l}\uparrow 20 \% \text { SOD } \\
\uparrow 40 \% \text { CAT } \\
\uparrow 50 \% \text { GR }\end{array}$ & $\uparrow 189 \%$ & \multirow[b]{2}{*}{ N/A } & $\uparrow 105 \%$ & & \\
\hline & $\begin{array}{l}125 \mathrm{mM} \mathrm{NaCl} \\
\text { + Unprimed }\end{array}$ & $\begin{array}{l}\downarrow 24 \% \mathrm{SL}, \downarrow 49 \% \mathrm{RL}, \downarrow 42 \% \mathrm{SDW}, \\
\downarrow 67 \% \mathrm{RDW}, \downarrow 48 \% \mathrm{SpDW}, \\
\downarrow 31 \% \mathrm{GY}, \downarrow 22 \% \mathrm{GPr}\end{array}$ & $\mathrm{N} / \mathrm{A}$ & $\begin{array}{l}\uparrow 23 \% \text { SOD } \\
\uparrow 80 \% \text { CAT } \\
\uparrow 75 \% \text { GR }\end{array}$ & $\uparrow 189 \%$ & & $\uparrow 146 \%$ & & \\
\hline
\end{tabular}


Table 2. Cont

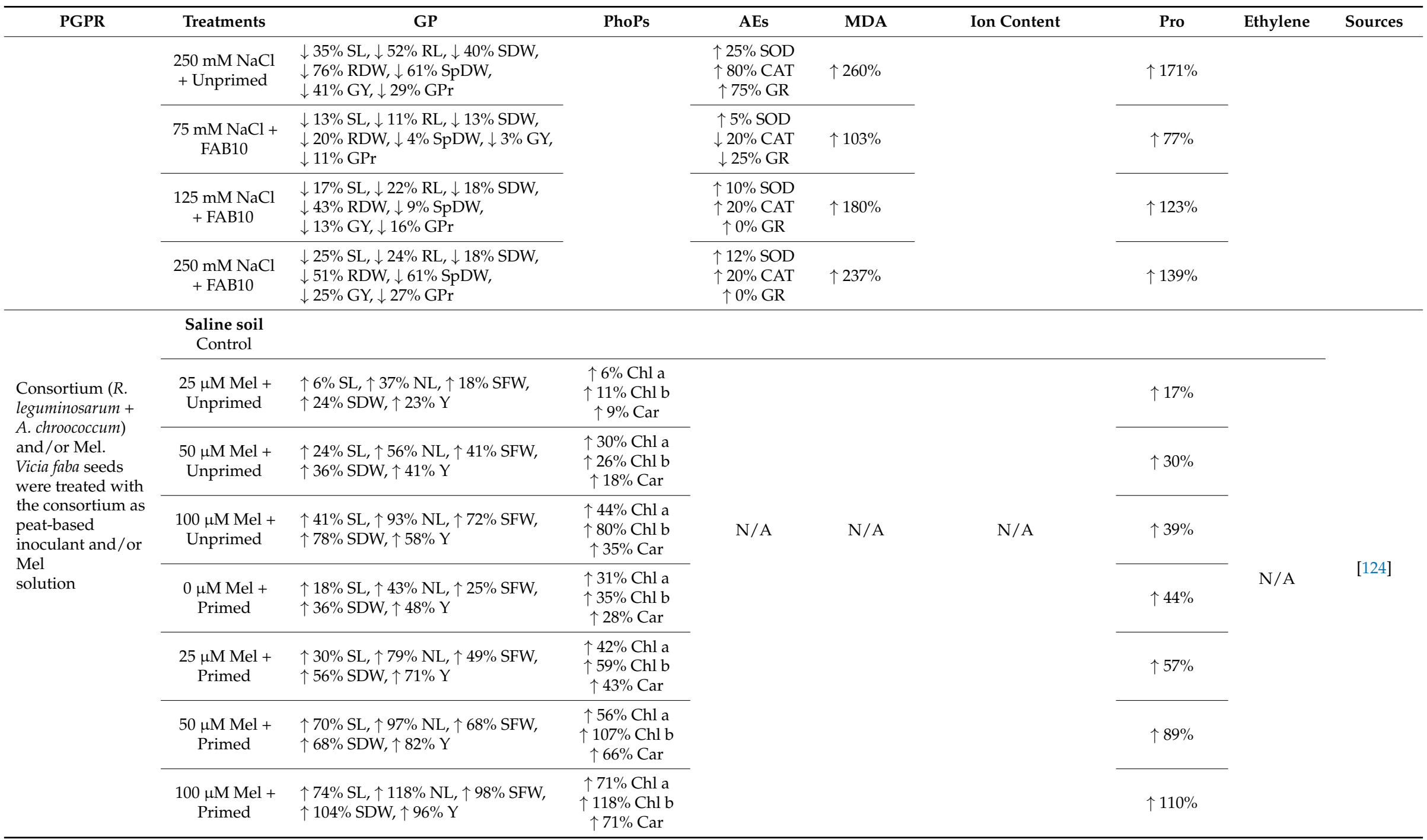


Table 2. Cont.

\begin{tabular}{|c|c|c|c|c|c|c|c|c|c|}
\hline PGPR & Treatments & GP & PhoPs & AEs & MDA & Ion Content & Pro & Ethylene & Sources \\
\hline & $\begin{array}{l}\text { Non-saline } \\
\text { soil Control }\end{array}$ & & & & & & & & \\
\hline & $\begin{array}{l}25 \mu \mathrm{M} \mathrm{Mel} \mathrm{+} \\
\text { Unprimed }\end{array}$ & $\begin{array}{l}\uparrow 6 \% \mathrm{SL}, \uparrow 14 \% \mathrm{NL}, \uparrow 10 \% \mathrm{SFW}, \\
\uparrow 21 \% \mathrm{SDW}, \uparrow 18 \% \mathrm{Y}\end{array}$ & $\begin{array}{l}\uparrow 9 \% \text { Chl a } \\
\uparrow 10 \% \text { Chl b } \\
\uparrow 4 \% \text { Car }\end{array}$ & & & & $\uparrow 5 \%$ & & \\
\hline & $\begin{array}{l}50 \mu \mathrm{M} \mathrm{Mel}+ \\
\text { Unprimed }\end{array}$ & $\begin{array}{l}\uparrow 12 \% \mathrm{SL}, \uparrow 35 \% \mathrm{NL}, \uparrow 23 \% \mathrm{SFW}, \\
\uparrow 31 \% \mathrm{SDW}, \uparrow 29 \% \mathrm{Y}\end{array}$ & $\begin{array}{l}\uparrow 11 \% \text { Chl a } \\
\uparrow 24 \% \text { Chl b } \\
\uparrow 21 \% \text { Car }\end{array}$ & & & & $\uparrow 11 \%$ & & \\
\hline & $\begin{array}{l}100 \mu \mathrm{M} \mathrm{Mel} \mathrm{+} \\
\text { Unprimed }\end{array}$ & $\begin{array}{l}\uparrow 16 \% \mathrm{SL}, \uparrow 49 \% \mathrm{NL}, \uparrow 38 \% \mathrm{SFW}, \\
\uparrow 69 \% \mathrm{SDW}, \uparrow 32 \% \mathrm{Y}\end{array}$ & $\begin{array}{l}\uparrow 16 \% \text { Chl a } \\
\uparrow 34 \% \text { Chl b } \\
\uparrow 24 \% \text { Car }\end{array}$ & & & & $\uparrow 45 \%$ & & \\
\hline & $\begin{array}{l}0 \mu \mathrm{MMel}+ \\
\text { Primed }\end{array}$ & $\begin{array}{l}\uparrow 20 \% \mathrm{SL}, \uparrow 28 \% \mathrm{NL}, \uparrow 14 \% \mathrm{SFW}, \\
\uparrow 27 \% \mathrm{SDW}, \uparrow 17 \% \mathrm{Y}\end{array}$ & $\begin{array}{l}\uparrow 17 \% \text { Chl a } \\
\uparrow 24 \% \text { Chl b } \\
\uparrow 21 \% \text { Car }\end{array}$ & & & & $\uparrow 36 \%$ & & \\
\hline & $\begin{array}{l}25 \mu \mathrm{M} \mathrm{Mel} \mathrm{+} \\
\text { Primed }\end{array}$ & $\begin{array}{l}\uparrow 29 \% \mathrm{SL}, \uparrow 49 \% \mathrm{NL}, \uparrow 24 \% \mathrm{SFW}, \\
\uparrow 46 \% \mathrm{SDW}, \uparrow 25 \% \mathrm{Y}\end{array}$ & $\begin{array}{l}\uparrow 23 \% \text { Chl a } \\
\uparrow 30 \% \text { Chl b } \\
\uparrow 30 \% \text { Car }\end{array}$ & & & & $\uparrow 94 \%$ & & \\
\hline & $\begin{array}{l}50 \mu \mathrm{M} \mathrm{Mel}+ \\
\quad \text { Primed }\end{array}$ & $\begin{array}{l}\uparrow 45 \% \mathrm{SL}, \uparrow 55 \% \mathrm{NL}, \uparrow 33 \% \mathrm{SFW}, \\
\uparrow 57 \% \mathrm{SDW}, \uparrow 38 \% \mathrm{Y}\end{array}$ & $\begin{array}{l}\uparrow 26 \% \mathrm{Chl} \mathrm{a} \\
\uparrow 42 \% \mathrm{Chl} \mathrm{b} \\
\uparrow 33 \% \mathrm{Car}\end{array}$ & & & & $\uparrow 110 \%$ & & \\
\hline & $\begin{array}{l}100 \mu \mathrm{M} \mathrm{Mel} \mathrm{+} \\
\text { Primed }\end{array}$ & $\begin{array}{l}\uparrow 49 \% \mathrm{SL}, \uparrow 76 \% \mathrm{NL}, \uparrow 52 \% \mathrm{SFW}, \\
\uparrow 87 \% \mathrm{SDW}, \uparrow 45 \% \mathrm{Y}\end{array}$ & $\begin{array}{l}\uparrow 30 \% \mathrm{Chl} \mathrm{a} \\
\uparrow 29 \% \mathrm{Chl} b \\
\uparrow 40 \% \mathrm{Car}\end{array}$ & & & & $\uparrow 139 \%$ & & \\
\hline \multirow{5}{*}{$\begin{array}{l}\text { A. aneurinilyticus } \\
\text { ACC02, } \\
\text { Paenibacillus } \\
\text { ACC06 and } \\
\text { Consortium } \\
\text { (ACC02+ ACC06). } \\
\text { French bean seeds } \\
\text { inoculated with } \\
\text { ACC02, } \\
\text { ACC06 and } \\
\text { consortium } \\
\text { (ACC02 + ACC06) }\end{array}$} & Control & & & & & & & & \multirow{5}{*}{ [88] } \\
\hline & $\underset{\mathrm{ACC} 02}{0 \mathrm{mM} \mathrm{NaCl}}+$ & $\begin{array}{l}\uparrow 10 \% \mathrm{SL}, \uparrow 50 \% \mathrm{RL}, \uparrow 158 \% \mathrm{SFW}, \\
\uparrow 10 \% \mathrm{SDW}, \uparrow 50 \% \mathrm{RFW}, \\
\uparrow 21 \% \mathrm{RDW}\end{array}$ & $\uparrow 36 \%$ Tchl & \multirow[t]{3}{*}{ N/A } & \multirow[t]{3}{*}{ N/A } & \multirow[t]{3}{*}{ N/A } & \multirow[t]{3}{*}{ N/A } & $\uparrow 9 \%$ & \\
\hline & $\underset{\mathrm{ACC} 06}{0 \mathrm{mM} \mathrm{NaCl}}+$ & $\begin{array}{l}\uparrow 30 \% \mathrm{SL}, \uparrow 30 \% \mathrm{RL}, \uparrow 216 \% \mathrm{SFW}, \\
\uparrow 10 \% \mathrm{SDW}, \uparrow 60 \% \mathrm{RFW}, \\
\uparrow 14 \% \mathrm{RDW}\end{array}$ & $\uparrow 29 \%$ Tchl & & & & & $\downarrow 9 \%$ & \\
\hline & $\begin{array}{l}0 \mathrm{mM} \mathrm{NaCl}+ \\
\text { Consortium }\end{array}$ & $\begin{array}{l}\uparrow 50 \% \mathrm{SL}, \uparrow 70 \% \mathrm{RL}, \uparrow 233 \% \mathrm{SFW}, \\
\uparrow 80 \% \mathrm{SDW}, \uparrow 90 \% \mathrm{RFW}, \\
\uparrow 85 \% \mathrm{RDW}\end{array}$ & $\uparrow 57 \%$ Tchl & & & & & $\uparrow 27 \%$ & \\
\hline & $\begin{array}{l}25 \mathrm{mM} \mathrm{NaCl}+ \\
\text { Unprimed }\end{array}$ & & & & & & & & \\
\hline
\end{tabular}


Table 2. Cont.

\begin{tabular}{|c|c|c|c|c|c|c|c|c|c|}
\hline PGPR & Treatments & GP & PhoPs & AEs & MDA & Ion Content & Pro & Ethylene & Sources \\
\hline & $\begin{array}{c}25 \mathrm{mM} \mathrm{NaCl} \\
\mathrm{ACC} 02\end{array}$ & $\begin{array}{l}\uparrow 33 \% \mathrm{SL}, \uparrow 79 \% \mathrm{RL}, \uparrow 120 \% \mathrm{SFW}, \\
\uparrow 300 \% \mathrm{SDW}, \uparrow 46 \% \mathrm{RFW}, \\
\uparrow 182 \% \mathrm{RDW}\end{array}$ & $\uparrow 28 \%$ Tchl & \multirow[t]{3}{*}{$\mathrm{N} / \mathrm{A}$} & \multirow[t]{3}{*}{ N/A } & \multirow[t]{3}{*}{ N/A } & \multirow[t]{3}{*}{ N/A } & $\downarrow 38 \%$ & \\
\hline & $\underset{\text { ACC06 }}{25 \mathrm{mM} \mathrm{NaCl}}+$ & $\begin{array}{l}\uparrow 47 \% \mathrm{SL}, \uparrow 58 \% \mathrm{RL}, \uparrow 120 \% \mathrm{SFW}, \\
\uparrow 350 \% \mathrm{SDW}, \uparrow 36 \% \mathrm{RFW}, \\
\uparrow 142 \% \mathrm{RDW}\end{array}$ & $\uparrow 35 \%$ Tchl & & & & & $\downarrow 42 \%$ & \\
\hline & $\begin{array}{c}25 \mathrm{mM} \mathrm{NaCl}+ \\
\text { Consortium }\end{array}$ & $\begin{array}{l}\uparrow 60 \% \mathrm{SL}, \uparrow 110 \% \mathrm{RL}, \uparrow 255 \% \mathrm{SFW}, \\
\uparrow 425 \% \mathrm{SDW}, \uparrow 81 \% \mathrm{RFW}, \\
\uparrow 220 \% \mathrm{RDW}\end{array}$ & $\uparrow 57 \%$ Tchl & & & & & $\downarrow 61 \%$ & \\
\hline \multirow{3}{*}{$\begin{array}{l}\text { P. fluorescence, } \\
\text { B. pumilus, } E \text {. } \\
\text { aurantiacum and } \\
\text { consortium }(P . \\
\text { fluorescence }+B \text {. } \\
\text { pumilus }+ \\
\text { E.aurantiacum }) \\
\text { Wheat seeds } \\
\text { soaked in } \\
\text { bacterial inoculant } \\
\text { containing single } \\
\text { PGPR strains or } \\
\text { the consortium of } \\
\text { three bacterial } \\
\text { cultures for } 2 \mathrm{~h} \text {. } \\
\text { Saline soil EC } \\
13.41\end{array}$} & $\begin{array}{l}\text { Unprimed } \\
\text { seeds }\end{array}$ & & & & & & & & \multirow{3}{*}{ [122] } \\
\hline & $\begin{array}{l}\text { Seeds primed } \\
\text { with } P \text {. } \\
\text { fluorescence }\end{array}$ & $\begin{array}{l}\text { Galaxy-13: } \\
\uparrow 5 \% \mathrm{SL}, \uparrow 7 \% \mathrm{RL}, \uparrow 3 \% \mathrm{SFW}, \\
\uparrow 2 \% \mathrm{SDW}, \uparrow 33 \% 100 \mathrm{GW}, \\
\downarrow 13 \% \mathrm{RFW}, \downarrow 29 \% \mathrm{RDW} \\
\text { Aas-11: } \\
\uparrow 11 \% \mathrm{SL}, \uparrow 24 \% \mathrm{RL}, \uparrow 48 \% \mathrm{SFW}, \\
\uparrow 144 \% \mathrm{RFW}, \uparrow 57 \% \mathrm{SDW}, \\
\uparrow 75 \% \mathrm{RDW}, \uparrow 23 \% 100 \mathrm{GW}\end{array}$ & $\mathrm{N} / \mathrm{A}$ & $\begin{array}{l}\text { Galaxy-13: } \\
\downarrow 30 \% \text { SOD } \\
\downarrow 0 \% \text { POD } \\
\uparrow 27 \% \text { CAT } \\
\text { Aas-11: } \\
\downarrow 57 \% \text { SOD } \\
\downarrow 14 \% \text { POD } \\
\uparrow 25 \% \text { CAT }\end{array}$ & \multirow[t]{2}{*}{$\mathrm{N} / \mathrm{A}$} & $\begin{array}{l}\text { Galaxy-13: } \\
\text { In root: } \\
\uparrow 50 \% \mathrm{Na}^{+}, \uparrow 40 \% \mathrm{~K}^{+}, \\
\mathrm{Na}^{+} / \mathrm{K}^{+} \text {ratio } 0.19 \\
\text { In shoot: } \uparrow 28 \% \mathrm{Na}^{+}, \uparrow \\
23 \% \mathrm{~K}^{+}, \mathrm{Na}^{+} / \mathrm{K}^{+} \text {ratio } \\
\sim 3.9 \\
\text { Aas-11: } \\
\text { In root: } \downarrow 13 \% \mathrm{Na}^{+}, \uparrow \\
99 \% \mathrm{~K}^{+}, \mathrm{Na}^{+} / \mathrm{K}^{+} \text {ratio } \\
\sim 0.16 . \\
\text { In shoot: } \uparrow 92 \% \mathrm{Na}^{+}, \uparrow \\
16 \% \mathrm{~K}^{+}, \mathrm{Na}^{+} / \mathrm{K}^{+} \text {ratio } \\
\sim 3.4\end{array}$ & $\begin{array}{c}\text { Galaxy-13: } \\
\downarrow 20 \% \\
\text { Aas-11: } \\
\uparrow 33 \%\end{array}$ & \multirow[t]{2}{*}{ N/A } & \\
\hline & $\begin{array}{l}\text { Seeds primed } \\
\text { with } B . \\
\text { pumilus }\end{array}$ & $\begin{array}{l}\text { Galaxy-13: } \\
\downarrow 7 \% \text { SL, } \downarrow 18 \% \text { SFW, } \downarrow 26 \% \text { SDW, } \\
\downarrow 8 \% \text { RFW, } \downarrow 57 \% \text { RDW, } \\
\uparrow 67 \% \text { RL, 31\% } 100 \mathrm{GW} \\
\text { Aas-11: } \\
\uparrow 13 \% \text { SL, } \uparrow 21 \% \text { RL, } \uparrow 61 \% \text { SFW, } \\
\uparrow 678 \% \text { RFW, } \uparrow 66 \% \text { SDW, } \\
\uparrow 838 \% \text { RDW, } \uparrow 53 \% 100 \mathrm{GW}\end{array}$ & & $\begin{array}{l}\text { Galaxy-13: } \\
\downarrow 35 \% \text { SOD } \\
\downarrow 5 \% \text { POD } \\
\uparrow 4 \% \text { CAT } \\
\text { Aas-11: } \\
\downarrow 65 \% \text { SOD } \\
\downarrow 38 \% \text { POD } \\
\uparrow 35 \% \text { CAT }\end{array}$ & & $\begin{array}{l}\text { Galaxy-13: } \\
\text { In root: } \uparrow 0 \% \mathrm{Na}^{+}, \uparrow 34 \% \\
\mathrm{~K}^{+}, \mathrm{Na}^{+} / \mathrm{K}^{+} \text {ratio } \sim 0.13 \\
\text { In shoot: } \downarrow 8 \% \mathrm{Na}^{+}, \downarrow \\
19 \% \mathrm{~K}^{+}, \mathrm{Na}^{+} / \mathrm{K}^{+} \text {ratio } \\
\sim 4.3 \\
\text { Aas-11: } \\
\text { In root: } \downarrow 13 \% \mathrm{Na}^{+}, \uparrow \\
195 \% \mathrm{~K}^{+}, \mathrm{Na}^{+} / \mathrm{K}^{+} \text {ratio } \\
\sim 0.11 \text {. } \\
\text { In shoot: } \uparrow 59 \% \mathrm{Na}^{+}, \downarrow \\
12 \% \mathrm{~K}^{+}, \mathrm{Na}^{+} / \mathrm{K}^{+} \text {ratio } \\
\sim 3.7\end{array}$ & $\begin{array}{c}\text { Galaxy-13: } \\
\text { 个2287\% } \\
\text { Aas-11: } \\
\uparrow 150 \%\end{array}$ & & \\
\hline
\end{tabular}


Table 2. Cont.

\begin{tabular}{|c|c|c|c|c|c|c|c|c|c|}
\hline PGPR & Treatments & GP & PhoPs & AEs & MDA & Ion Content & Pro & Ethylene & Sources \\
\hline & $\begin{array}{l}\text { Seeds primed } \\
\text { with } \\
\text { E.aurantiacum }\end{array}$ & $\begin{array}{l}\text { Galaxy-13: } \\
\uparrow 6 \% \mathrm{SL}, \uparrow 47 \% \mathrm{RL}, \uparrow 3 \% \mathrm{SFW}, \\
\uparrow 49 \% 100 \mathrm{GW}, \downarrow 17 \% \mathrm{RFW}, \\
\downarrow 2 \% \mathrm{SDW}, \downarrow 28 \% \mathrm{RDW} \\
\text { Aas-11: } \\
\uparrow 10 \% \mathrm{SL}, \uparrow 7 \% \mathrm{RL}, \uparrow 52 \% \mathrm{SFW}, \\
\uparrow 511 \% \mathrm{RFW}, \uparrow 71 \% \mathrm{SDW}, \\
\uparrow 713 \% \mathrm{RDW}, \uparrow 47 \% 100 \mathrm{GW}\end{array}$ & & $\begin{array}{l}\text { Galaxy-13: } \\
\uparrow 2 \% \text { SOD } \\
\uparrow 48 \% \text { CAT } \\
\downarrow 43 \% \text { POD } \\
\text { Aas-11: } \\
\downarrow 65 \% \text { SOD } \\
\downarrow 57 \% \text { POD } \\
\downarrow 5 \% \text { CAT }\end{array}$ & & $\begin{array}{l}\text { Galaxy-13: } \\
\text { In root: } \uparrow 33 \% \mathrm{Na}^{+}, \uparrow \\
34 \% \mathrm{~K}^{+}, \mathrm{Na}^{+} / \mathrm{K}^{+} \text {ratio } \\
\sim 0.18 \\
\text { In shoot: } \uparrow 27 \% \mathrm{Na}^{+}, \uparrow \\
0 \% \mathrm{~K}^{+}, \mathrm{Na}^{+} / \mathrm{K}^{+} \text {ratio } \\
\sim 4.77 . \\
\text { Aas-11: } \\
\text { In root: } \downarrow 13 \% \mathrm{Na}^{+}, \uparrow \\
286 \% \mathrm{~K}^{+}, \mathrm{Na}^{+} / \mathrm{K}^{+} \text {ratio } \\
\sim 0.08 \\
\text { In shoot: } \uparrow 40 \% \mathrm{Na}^{+}, \uparrow \\
22 \% \mathrm{~K}^{+}, \mathrm{Na}^{+} / \mathrm{K}^{+} \text {ratio } \\
\sim 2.36\end{array}$ & $\begin{array}{c}\text { Galaxy-13: } \\
\quad \uparrow 227 \% \\
\text { Aas- } 11: \\
\uparrow 110 \%\end{array}$ & & \\
\hline & $\begin{array}{l}\text { Seeds primed } \\
\text { with a } \\
\text { consortium }\end{array}$ & $\begin{array}{l}\text { Galaxy-13: } \\
\downarrow 1 \% \mathrm{SL}, \uparrow 73 \% \mathrm{RL}, \uparrow 6 \% \mathrm{SFW}, \\
\uparrow 30 \% \mathrm{RFW}, \uparrow 7 \% \mathrm{SDW}, \\
\uparrow 43 \% \mathrm{RDW}, \uparrow 53 \% 100 \mathrm{GW} \\
\text { Aas-11: } \\
\uparrow 13 \% \mathrm{SL}, \uparrow 3 \% \mathrm{RL}, \uparrow 65 \% \mathrm{SFW}, \\
\uparrow 556 \% \mathrm{RFW}, \uparrow 77 \% \mathrm{SDW}, \\
\uparrow 725 \% \mathrm{RDW}, \uparrow 48 \% 100 \mathrm{GW}\end{array}$ & & $\begin{array}{l}\text { Galaxy-13: } \\
\uparrow 37 \% \text { SOD } \\
\downarrow 32 \% \text { POD } \\
\downarrow 6 \% \text { CAT } \\
\text { Aas-11: } \\
\downarrow 57 \% \text { SOD } \\
\uparrow 24 \% \text { POD } \\
\uparrow 28 \% \text { CAT }\end{array}$ & & $\begin{array}{l}\text { Galaxy-13: } \\
\text { In root: } \uparrow 0 \% \mathrm{Na}^{+}, \uparrow \\
114 \% \mathrm{~K}^{+}, \mathrm{Na}^{+} / \mathrm{K}^{+} \text {ratio } \\
\sim 0.08 \\
\text { In shoot: } \uparrow 17 \% \mathrm{Na}^{+}, \uparrow \\
15 \% \mathrm{~K}^{+}, \mathrm{Na}^{+} / \mathrm{K}^{+} \text {ratio } \\
\sim 3.8 \\
\text { Aas-11: } \\
\text { In root: } \uparrow 0 \% \mathrm{Na}^{+}, \uparrow \\
173 \% \mathrm{~K}^{+}, \mathrm{Na}^{+} / \mathrm{K}^{+} \text {ratio } \\
\sim 0.13 \\
\text { In shoot: } \uparrow 68 \% \mathrm{Na}^{+}, \uparrow \\
30 \% \mathrm{~K}^{+}, \mathrm{Na}^{+} / \mathrm{K}^{+} \text {ratio } \\
\sim 2.67\end{array}$ & $\begin{array}{c}\text { Galaxy-13: } \\
\uparrow 327 \% \\
\text { Aas-11: } \\
\uparrow 17 \%\end{array}$ & & \\
\hline \multirow{2}{*}{$\begin{array}{l}\text { Sphingobacterium } \\
\text { BHU-AV3. } \\
\text { Bacterized tomato } \\
\text { seeds with } \\
\text { BHU-AV3 for } 24 \mathrm{~h}\end{array}$} & Control & & & & & & & & \\
\hline & $\begin{array}{l}200 \mathrm{mM} \mathrm{NaCl} \\
+ \text { Unprimed }\end{array}$ & $\begin{array}{l}\downarrow 52 \% \mathrm{SL}, \downarrow 49 \% \mathrm{RL}, \\
\downarrow 54 \% \mathrm{TPDW}\end{array}$ & $\downarrow 44 \%$ Tchl & $\begin{array}{c}\text { In shoot: } \\
\uparrow 90 \% \text { SOD } \\
\uparrow 260 \% \text { POD } \\
\uparrow 100 \% \text { PPO } \\
\text { In root: } \\
\uparrow 83 \% \text { SOD } \\
\uparrow 100 \% \text { POD } \\
\uparrow 53 \% \text { PPO }\end{array}$ & N/A & $\begin{array}{l}\text { In shoot: } \uparrow 258 \% \mathrm{Na}^{+}, \downarrow \\
63 \% \mathrm{~K}^{+}, \mathrm{Na}^{+} / \mathrm{K}^{+} \text {ratio } \\
\sim 3.6 \\
\text { In root: } \uparrow 190 \% \mathrm{Na}^{+}, \downarrow \\
53 \% \mathrm{~K}^{+}, \mathrm{Na}^{+} / \mathrm{K}^{+} \text {ratio } \\
\sim 3.5\end{array}$ & $\begin{array}{c}\text { In shoot: } \\
\uparrow 153 \% \\
\text { In root: } \\
\uparrow 56 \%\end{array}$ & N/A & [47] \\
\hline
\end{tabular}


Table 2. Cont.

\begin{tabular}{|c|c|c|c|c|c|c|c|c|c|}
\hline PGPR & Treatments & GP & PhoPs & AEs & MDA & Ion Content & Pro & Ethylene & Sources \\
\hline & $\begin{array}{c}0 \mathrm{mM} \mathrm{NaCl}+ \\
\text { BHU-AV3 }\end{array}$ & $\begin{array}{l}\downarrow 11.3 \% \mathrm{SL}, \uparrow 16 \% \mathrm{RL}, \\
\uparrow 11 \% \mathrm{TPDW}\end{array}$ & $\uparrow 5 \%$ Tchl & $\begin{array}{l}\text { In shoot: } \\
\downarrow 20 \% \text { SOD } \\
\downarrow 0 \% \text { POD } \\
\downarrow 25 \% \text { PPO } \\
\text { In root: } \\
\uparrow 16 \% \text { SOD } \\
\uparrow 6 \% \text { POD } \\
\downarrow 12 \% \text { PPO }\end{array}$ & & $\begin{array}{l}\text { In shoot: } \uparrow 9 \% \mathrm{Na}^{+}, \uparrow 9 \% \\
\mathrm{~K}^{+}, \mathrm{Na}^{+} / \mathrm{K}^{+} \text {ratio } \sim 0.3 \\
\text { In root: } \downarrow 5 \% \mathrm{Na}^{+}, \uparrow 8 \% \\
\mathrm{~K}^{+}, \mathrm{Na}^{+} / \mathrm{K}^{+} \text {ratio } \sim 0.5\end{array}$ & $\begin{array}{c}\text { In shoot: } \\
\downarrow 7 \% \\
\text { In root: } \\
\downarrow 5 \%\end{array}$ & & \\
\hline & $\begin{array}{l}200 \mathrm{mM} \mathrm{NaCl} \\
+ \text { BHU-AV3 }\end{array}$ & $\begin{array}{l}\downarrow 30 \% \text { SL }, \downarrow 22 \% \text { RL } \\
\downarrow 29 \% \text { TPDW }\end{array}$ & $\downarrow 14 \%$ Tchl & $\begin{array}{c}\text { In shoot: } \\
\uparrow 10 \% \text { SOD } \\
\uparrow 1000 \% \text { POD } \\
\uparrow 50 \% \text { PPO } \\
\text { In root: } \\
\uparrow 117 \% \text { SOD } \\
\uparrow 200 \% \text { POD } \\
\uparrow 71 \% \text { PPO }\end{array}$ & & $\begin{array}{l}\text { In shoot: } \uparrow 130 \% \mathrm{Na}^{+}, \downarrow \\
20 \% \mathrm{~K}^{+}, \mathrm{Na}^{+} / \mathrm{K}^{+} \text {ratio } \\
\sim 1 \\
\text { In root: } \uparrow 115 \% \mathrm{Na}^{+}, \downarrow \\
24 \% \mathrm{~K}^{+}, \mathrm{Na}^{+} / \mathrm{K}^{+} \text {ratio } \\
\sim 1.6\end{array}$ & $\begin{array}{l}\text { In shoot: } \\
\uparrow 84 \% \\
\text { In root: } \\
\uparrow 111 \%\end{array}$ & & \\
\hline \multirow{6}{*}{$\begin{array}{l}\text { K. sacchari MSK1. } \\
\text { Mung bean seeds } \\
\text { primed with } \\
\text { MSK1 }\end{array}$} & Control & & & & & & & \multirow{6}{*}{$\mathrm{N} / \mathrm{A}$} & \multirow{6}{*}{ [36] } \\
\hline & $\begin{array}{l}50 \mathrm{mM} \mathrm{NaCl}+ \\
\text { Unprimed }\end{array}$ & $\begin{array}{l}\downarrow 8 \% \text { SL, } \downarrow 8 \% \text { RL, } \downarrow 5 \% \text { SDW, } \\
\downarrow 5 \% \text { RDW, } \downarrow 15 \% \text { SY, } \downarrow 3 \% \text { GP }\end{array}$ & $\begin{array}{l}\downarrow 15 \% \text { Tchl } \\
\downarrow 4 \% \text { Car }\end{array}$ & $\begin{array}{r}\uparrow 5 \% \mathrm{GR}, \\
\uparrow 33 \% \mathrm{CAT} \\
\uparrow 13 \% \mathrm{SOD} \\
\uparrow 23 \% \mathrm{APX} \\
\end{array}$ & $\uparrow 32 \%$ & $\begin{array}{l}\text { In shoor: } \uparrow 100 \% \mathrm{Na}^{+}, \uparrow \\
44 \% \mathrm{~K}^{+}, \mathrm{Na}^{+} / \mathrm{K}^{+} \text {ratio } \\
\sim 0.46, \downarrow 4 \% \mathrm{~N}, \\
\downarrow 19 \% \mathrm{P}\end{array}$ & $\uparrow 63 \%$ & & \\
\hline & $\begin{array}{l}100 \mathrm{mM} \mathrm{NaCl} \\
+ \text { Unprimed }\end{array}$ & $\begin{array}{l}\downarrow 16 \% \text { SL, } \downarrow 20 \% \text { RL, } \downarrow 10 \% \text { SDW, } \\
\downarrow 15 \% \text { RDW, } \downarrow 21 \% \text { SY, } \downarrow 6 \% \text { GP }\end{array}$ & $\begin{array}{l}\downarrow 35 \% \text { Tchl } \\
\downarrow 9 \% \text { Car }\end{array}$ & $\begin{array}{l}\uparrow 15 \% \text { GR } \\
\uparrow 58 \% \text { CAT } \\
\uparrow 39 \% \text { SOD } \\
\uparrow 45 \% \text { APX }\end{array}$ & $\uparrow 47 \%$ & $\begin{array}{l}\text { In shoot: } \uparrow 200 \% \mathrm{Na}^{+}, \uparrow \\
100 \% \mathrm{~K}^{+}, \mathrm{Na}^{+} / \mathrm{K}^{+} \text {ratio } \\
\sim 0.5, \downarrow 4 \% \mathrm{~N}, \\
\downarrow 28 \% \mathrm{P}\end{array}$ & $\uparrow 88 \%$ & & \\
\hline & $\begin{array}{l}200 \mathrm{mM} \mathrm{NaCl} \\
\text { + Unprimed }\end{array}$ & $\begin{array}{l}\downarrow 24 \% \mathrm{SL}, \downarrow 28 \% \mathrm{RL}, \downarrow 21 \% \mathrm{SDW}, \\
\downarrow 35 \% \mathrm{RDW}, \downarrow 26 \% \mathrm{SY}, \downarrow 8 \% \mathrm{GP}\end{array}$ & $\begin{array}{l}\downarrow 42 \% \text { Tchl } \\
\downarrow 19 \% \text { Car }\end{array}$ & $\begin{array}{c}\uparrow 35 \% \text { GR } \\
\uparrow 108 \% \text { CAT } \\
\uparrow 52 \% \text { SOD } \\
\uparrow 73 \% \text { APX }\end{array}$ & $\uparrow 84 \%$ & $\begin{array}{l}\text { In shoot: } \uparrow 450 \% \mathrm{Na}^{+}, \uparrow \\
222 \% \mathrm{~K}^{+}, \mathrm{Na}^{+} / \mathrm{K}^{+} \text {ratio } \\
\sim 0.57, \downarrow 12 \% \mathrm{~N}, \\
\downarrow 44 \% \mathrm{P}\end{array}$ & $\uparrow 213 \%$ & & \\
\hline & $\begin{array}{l}400 \mathrm{mM} \mathrm{NaCl} \\
\text { + Unprimed }\end{array}$ & $\begin{array}{l}\downarrow 41 \% \mathrm{SL}, \downarrow 52 \% \mathrm{RL}, \downarrow 34 \% \mathrm{SDW}, \\
\downarrow 55 \% \mathrm{RDW}, \downarrow 34 \% \mathrm{SY}, \downarrow 26 \% \mathrm{GP}\end{array}$ & $\begin{array}{l}\downarrow 62 \% \text { Tchl } \\
\downarrow 33 \% \text { Car }\end{array}$ & $\begin{array}{l}\uparrow 64 \% \text { GR } \\
\uparrow 208 \% \text { CAT } \\
\uparrow 96 \% \text { SOD } \\
\uparrow 102 \% \text { APX }\end{array}$ & $\uparrow 153 \%$ & $\begin{array}{l}\text { In shoot: } \uparrow 800 \% \mathrm{Na}^{+}, \uparrow \\
378 \% \mathrm{~K}^{+}, \mathrm{Na}^{+} / \mathrm{K}^{+} \text {ratio } \\
\sim 0.63, \downarrow 21 \% \mathrm{~N}, \\
\downarrow 59 \% \mathrm{P}\end{array}$ & $\uparrow 350 \%$ & & \\
\hline & $\begin{array}{c}0 \mathrm{mM} \mathrm{NaCl} \\
\mathrm{MSK} 1\end{array}$ & $\begin{array}{l}\uparrow 5 \% \mathrm{SL}, \uparrow 12 \% \mathrm{RL}, \uparrow 7 \% \mathrm{SDW}, \\
\uparrow 15 \% \mathrm{RDW}, \uparrow 9 \% \mathrm{SY}, \uparrow 7 \% \mathrm{GP}\end{array}$ & $\begin{array}{l}\uparrow 29 \% \text { Tchl } \\
\uparrow 7 \% \text { Car }\end{array}$ & $\begin{array}{l}\downarrow 9 \% \text { GR } \\
\downarrow 33 \% \text { CAT } \\
\downarrow 22 \% \text { SOD } \\
\downarrow 9 \% \text { APX }\end{array}$ & $\downarrow 37 \%$ & $\begin{array}{l}\text { In shoot: } \downarrow 67 \% \mathrm{Na}^{+}, \downarrow \\
22 \% \mathrm{~K}^{+}, \mathrm{Na}^{+} / \mathrm{K}^{+} \text {ratio } \\
\sim 0.14, \uparrow 9 \% \mathrm{~N}, \\
\uparrow 15 \% \mathrm{P}\end{array}$ & $\downarrow 25 \%$ & & \\
\hline
\end{tabular}


Table 2. Cont.

\begin{tabular}{|c|c|c|c|c|c|c|c|c|c|}
\hline PGPR & Treatments & GP & PhoPs & AEs & MDA & Ion Content & Pro & Ethylene & Sources \\
\hline & $\begin{array}{l}50 \mathrm{mM} \mathrm{NaCl}+ \\
\mathrm{MSK} 1\end{array}$ & $\begin{array}{l}\downarrow 3 \% \mathrm{SL}, \uparrow 4 \% \mathrm{RL}, \downarrow 2 \% \mathrm{SDW}, \\
\uparrow 3 \% \mathrm{RDW}, \uparrow 10 \% \mathrm{SY}, \uparrow 2 \% \mathrm{GP}\end{array}$ & $\begin{array}{l}\downarrow 3 \% \text { Tchl } \\
\downarrow 0 \% \text { Car }\end{array}$ & $\begin{array}{l}\uparrow 2 \% \text { GR } \\
\uparrow 8 \% \text { CAT } \\
\uparrow 9 \% \text { SOD } \\
\uparrow 9 \% \text { APX }\end{array}$ & $\uparrow 11 \%$ & $\begin{array}{l}\text { In shoot: } \uparrow 33 \% \mathrm{Na}^{+}, \uparrow \\
22 \% \mathrm{~K}^{+}, \mathrm{Na}^{+} / \mathrm{K}^{+} \text {ratio } \\
\sim 0.36, \downarrow 1 \% \mathrm{~N}, \\
\downarrow 41 \% \mathrm{P}\end{array}$ & $\uparrow 30 \%$ & & \\
\hline & $\begin{array}{l}100 \mathrm{mM} \mathrm{NaCl} \\
+ \text { MSK1 }\end{array}$ & $\begin{array}{l}\downarrow 8 \% \mathrm{SL}, \downarrow 12 \% \mathrm{RL}, \downarrow 7 \% \mathrm{SDW}, \\
\downarrow 10 \% \mathrm{RDW}, \downarrow 19 \% \mathrm{SY}, \downarrow 5 \% \mathrm{GP}\end{array}$ & $\begin{array}{l}\downarrow 31 \% \text { Tchl } \\
\downarrow 8 \% \text { Car }\end{array}$ & $\begin{array}{l}\uparrow 11 \% \text { GR } \\
\uparrow 50 \% \text { CAT } \\
\uparrow 22 \% \text { SOD } \\
\uparrow 32 \% \text { APX }\end{array}$ & $\uparrow 37 \%$ & $\begin{array}{l}\text { In shoot: } \uparrow 183 \% \mathrm{Na}^{+}, \uparrow \\
89 \% \mathrm{~K}^{+}, \mathrm{Na}^{+} / \mathrm{K}^{+} \text {ratio } \\
\sim 0.5, \downarrow 4 \% \mathrm{~N}, \\
\downarrow 22 \% \mathrm{P}\end{array}$ & $\uparrow 75 \%$ & & \\
\hline & $\begin{array}{l}200 \mathrm{mM} \mathrm{NaCl} \\
+ \text { MSK1 }\end{array}$ & $\begin{array}{l}\downarrow 22 \% \text { SL, } \downarrow 16 \% \text { RL, } \downarrow 17 \% \text { SDW, } \\
\downarrow 25 \% \text { RDW, } \downarrow 24 \% \text { SY, } \downarrow 7 \% \text { GP }\end{array}$ & $\begin{array}{l}\downarrow 35 \% \text { Tchl } \\
\downarrow 13 \% \text { Car }\end{array}$ & $\begin{array}{c}\uparrow 33 \% \text { GR } \\
\uparrow 100 \% \text { CAT } \\
\uparrow 48 \% \text { SOD } \\
\uparrow 64 \% \text { APX }\end{array}$ & $\uparrow 79 \%$ & $\begin{array}{c}\text { In shoot: } \uparrow 433 \% \mathrm{Na}^{+}, \uparrow \\
211 \% \mathrm{~K}^{+}, \mathrm{Na}^{+} / \mathrm{K}^{+} \text {ratio } \\
\sim 0.57, \downarrow 9 \% \mathrm{~N}, \\
\downarrow 37 \% \mathrm{P}\end{array}$ & $\uparrow 200 \%$ & & \\
\hline & $\begin{array}{l}400 \mathrm{mM} \mathrm{NaCl} \\
+ \text { MSK1 }\end{array}$ & $\begin{array}{l}\downarrow 35 \% \text { SL, } \downarrow 24 \% \text { RL, } \downarrow 32 \% \text { SDW, } \\
\downarrow 48 \% \text { RDW }, \downarrow 32 \% \text { SY, } \downarrow 25 \% \text { GP }\end{array}$ & $\begin{array}{l}\downarrow 54 \% \text { Tchl } \\
\downarrow 27 \% \text { Car }\end{array}$ & $\begin{array}{l}\uparrow 60 \% \text { GR } \\
\uparrow 192 \% \text { CAT } \\
\uparrow 91 \% \text { SOD } \\
\uparrow 91 \% \text { APX }\end{array}$ & $\uparrow 137 \%$ & $\begin{array}{l}\text { In shoot: } \uparrow 783 \% \mathrm{Na}^{+}, \uparrow \\
367 \% \mathrm{~K}^{+}, \mathrm{Na}^{+} / \mathrm{K}^{+} \text {ratio } \\
\sim 0.63, \downarrow 19 \% \mathrm{~N}, \\
\downarrow 57 \% \mathrm{P}\end{array}$ & $\uparrow 325 \%$ & & \\
\hline
\end{tabular}

Note: All calculations in the Table 2 represent the comparisons between the treated plants and the control plants (non-stress conditions and un-priming) except that the control plants in the study of Nawaz et al. (2020) [122] were cultivated in the saline soil ECe $~ 13.41$. The up arrowhead ( $\uparrow$ ) indicates an increase in a tested parameter as compared to the control. The down arrowhead $(\downarrow)$ displays a reduction in a tested parameter relative to the control. Abbreviation in the Table 2: A. calcoaceticus, Acinetobacter calcoaceticus; A. aneurinilyticus, Aneurinibacillus aneurinilyticus; A chroococcum, Azotobacter chroococcum; AEs, Antioxidant enzymes; APX, Ascorbate peroxidase; B. pumilus, Bacillus pumilus; Car, Carotenoids; CAT, Catalase; Chl, Chlorophyll; E. aurantiacum, Exiguobacterium aurantiacum; GP, Germination percentage; GPr, Grain protein; GR, Glutathione reductase; GW, Grain weight; GY, Grain yield; K. sacchari, Kosakonia sacchari; MDA, Malondialdehyde; Mel, Melatonin; N/A, Not available; NL, Number of leaves per plant; P. fluorescence, Pseudomonas fluorescence; PhoPs, Photosynthetic pigments; POD, Peroxidase; P. putida, Pseudomonas putida; PPO, Polyphenol oxidase; RDW, Root dry weight; RFW, Root fresh weight; R. leguminosarum, Rhizobium leguminosarum; RL, Root length; SDW, Shoot dry weight; SFW, Shoot fresh weight; SL, Shoot length; S. maltophilia, Stenotrophomonas maltophilia; SOD, Superoxide dismutase; SpDW, Spike dry weight; SVI, Seedling vigor index; SY, Seed yield; Tchl, Total chlorophyll; TPDW, Total plant dry weight; Y, Yield. 
3.3. The Reduction in AEs and OS in PGPR-Inoculated Plants and PGPR-Primed Seedlings under Salt Stress

The changes in AEs and osmo-regulators have been noticed in both uninoculatedand inoculated plants under normal and salinity conditions. However, the reduction or increase of these enzymes in PGPB-inoculated plants in response to salt conditions remains controversial. The decreased profiles of OS and/or ROS-scavenging enzymes were remarked by Kang et al. (2014 a) [43], Kang et al. (2014 b) [41], Barnawal et al. (2014) [95], Khan et al. (2016) [40], Bhise et al. (2017) [99], Abd_Allah et al. (2018) [35], Sapre et al. (2018) [44], Ansari et al. (2019) [51], Alexander et al. (2020) [48], Misra and Chauhan (2020) [106], and Shahid et al. (2021) [36]. Specifically, in the study of Kang et al. (2014a) [43], the activities of CAT, PPO, and POD enzymes and the PPs contents in the inoculated plants (e.g., B. cepacia SE4, Promicromonospora sp. SE188 or A. calcoaceticus SE370) were lower than those in the uninoculated plants under salt stress (120 mM of $\mathrm{NaCl}$ ). The reduced profiles of AEs in Kang and his colleagues' findings were in agreement with their another study on soybean using the bacterium P. putida H-2-3 [41] and also in line with the study of Sapre et al. [44]. According to Sapre and colleagues' finding, the Klebsiella-treated wheat plants increased by $96 \%$ SOD and $286 \%$ POD, while the SOD and POD in untreated plants were increased by $353 \%$ and $540 \%$, respectively. These data were in agreement with those found by Shahid et al. [36], and Sarkar et al. [49] as these investigators found that the highest antioxidant enzyme activities were recorded in the non-inoculated mung bean and rice plants, respectively. In parallel with the report of Sarkar et al. [49], Rojas-Tapias et al. [128] also recorded the highest Pro content was found in the non-inoculated maize seedling leaves under salt stress. The increase of PP contents in bacterized plants was also recorded in $[41,43]$, however, to a lesser extent than those in the untreated plants. Similarly, Pro accumulations in the tissues of the control oat plants and the control rice plants were much higher than those in the Klebsiella-inoculated oat plants and Entorobacter-inoculated rice plants (230\% and $175 \%$, respectively vs. $155 \%$ and $75 \%$, respectively) [44,49]. These studies showed similar findings with Manaf and Zayed [158] as the SOD activity and proline content in the cowpea plants treated with mycorrhizae or P. fluorescence alone were lower than those in the untreated plants under $3000 \mathrm{ppm}$ $\mathrm{NaCl}$ irrigation regime. Manaf and Zayed assumed that the harmful effects of high salinity made the plants lose the ability to control their metabolites [158], whereas Sapre et al. [44] speculated that the treated plants did not sense much stress as the untreated plants did, leading the lower levels of AEs, NEAs, and osmoregulators in their tissues. Misra and Chauhan [106] proposed that the reduced Pro and AEs in Bacillus-treated maize plants may be due to the formation of EPS and biofilm on plant root surfaces that prevented plants from over-uptake $\mathrm{Na}^{+}$, thereby attenuating the detrimental effects of toxic ions on plants. This assumption was corroborated by a study of Mukherjee et al. [110], who found that the amount of EPS-bound $\mathrm{Na}^{+}$increased with the increase in $\mathrm{NaCl}$ concentration in the solution, thus confirming an efficient role of EPS in $\mathrm{NaCl}$ sequestration. In addition, Sarkar et al. [49] explained that the increased antioxidant enzyme activities of Enterobacter sp. P23 under saline stress could indirectly quench a significant amount of ROS in rice seedlings, thus delaying the urge to synthesize ROS scavengers by stressed plants.

\subsection{Genetic Diversities of Plant and Microbe, Plant-Microbe Interactions and Microbe-Microbe} Interactions Are Key Players in Regulating AEs Profiles

In the first case where different plant species inoculated with PGPR species from the same genus Curtobacterium, the reduced PPO and POD activities were observed in the Curtobacterium oceanosedimentum SAK1-treated soybean plants [159], whereas the increase in POD, CAT, SOD, and APX were recorded in the Curtobacterium albidum SRV4-treated paddy plants [154].

In the second situation, soybean plants inoculated with different PGPR species also revealed the contrasting antioxidant enzyme profiles. For instance, the soybean plants cv. Giza 35 treated with Bacillus firmus SW5 showed a significant increase in APX, SOD, CAT, and POD activities [153], whereas the SOD and DPPH scavenging activities were 
relatively decreased in the soybean plants cv. Taekwang inoculated with Pseudomonas putida $\mathrm{H}-2-3$ [41]. Moreover, variation in enzyme activities were found in the maize variety cv. Maharaja inoculated with different PGPR species [106]. Specifically, the maize seedlings Maharaja treated with Bacillus subtilis (NBNI 28B) had higher GPX and CAT as compared to the control, whereas the seedlings treated with B. subtilis (NBRI $33 \mathrm{~N}$ ) and B. safensis (NBRI $12 \mathrm{M}$ ) exhibited lower SOD, APX, GPX, CAT, and PPO than those in the control and those in the NBNI 28B-bacterized seedlings [106].

Regarding the effect of consortium treatment on AEs, the POD activity in the saltsensitive wheat genotype Galaxy-13 inoculated with individual Pseudomonas fluorescence, Bacillus pumilus, and Exiguobacterium aurantiacum was always higher than that in the salt-tolerant wheat genotype Aas-11 [122]. The effect of the consortium (Pseudomonas fluorescence, Bacillus pumilus, and Exiguobacterium aurantiacum), however, resulted in the lower POD activity in the treated Galaxy-13 in respect of the treated Aas-11. Similarly, CAT activity was higher in the Galaxy-13 treated alone with P. fluorescence and E. aurantiacum, in comparison with that in Aas-11, but the CAT activity of Galaxy-13 was lower than that of Aas-11 in the consortium treatment. In contrast, Galaxy-13 had lower SOD activity in a single inoculation with P. fluorescence and B. pumilus, but it exhibited higher SOD activity than Aas-11 in the consortium treatment.

It is worthy to note that antioxidant response to salinity was varied in different cultivars in the same plant species. Kharusi et al. (2019) [160] noticed that the salt-tolerant date palm cultivar Umsila maintained a normal concentration of ROS by accumulating elevated NEAs and by stimulating greater AEs activities with respect to the salt-sensitive date palm cultivar Zabad. The activities of SOD, CAT, APX and the contents of GSH, FLA, PCs, and Pro in Umsila were statistically significantly greater than those in Zabad when exposed to salt stress.

In summary, the findings in these previous studies, taken together, suggest that an increase or a reduction in the activities of AEs and/or OS in PGPR-inoculated plants during salt stress adaptation depends mainly on the specificities of plant species, on PGPR species, interactions between PGPR in consortia, and on plant-microbe interactions. These controversial data indicate not only that the fine-tuning of the ROS quenchers might be critical for plants to tolerate better to salt stress, but also pose questions concerning the exact mechanisms of salt stress tolerance imposed by PGPR. So far, investigators mainly based on their personal assumption, but not on scientific evidence, to elucidate the fluctuation in AEs. Integrated Omics approach would be necessary to gain insight into this interesting issue. The main message of the present review was displayed in the Figure 1. 


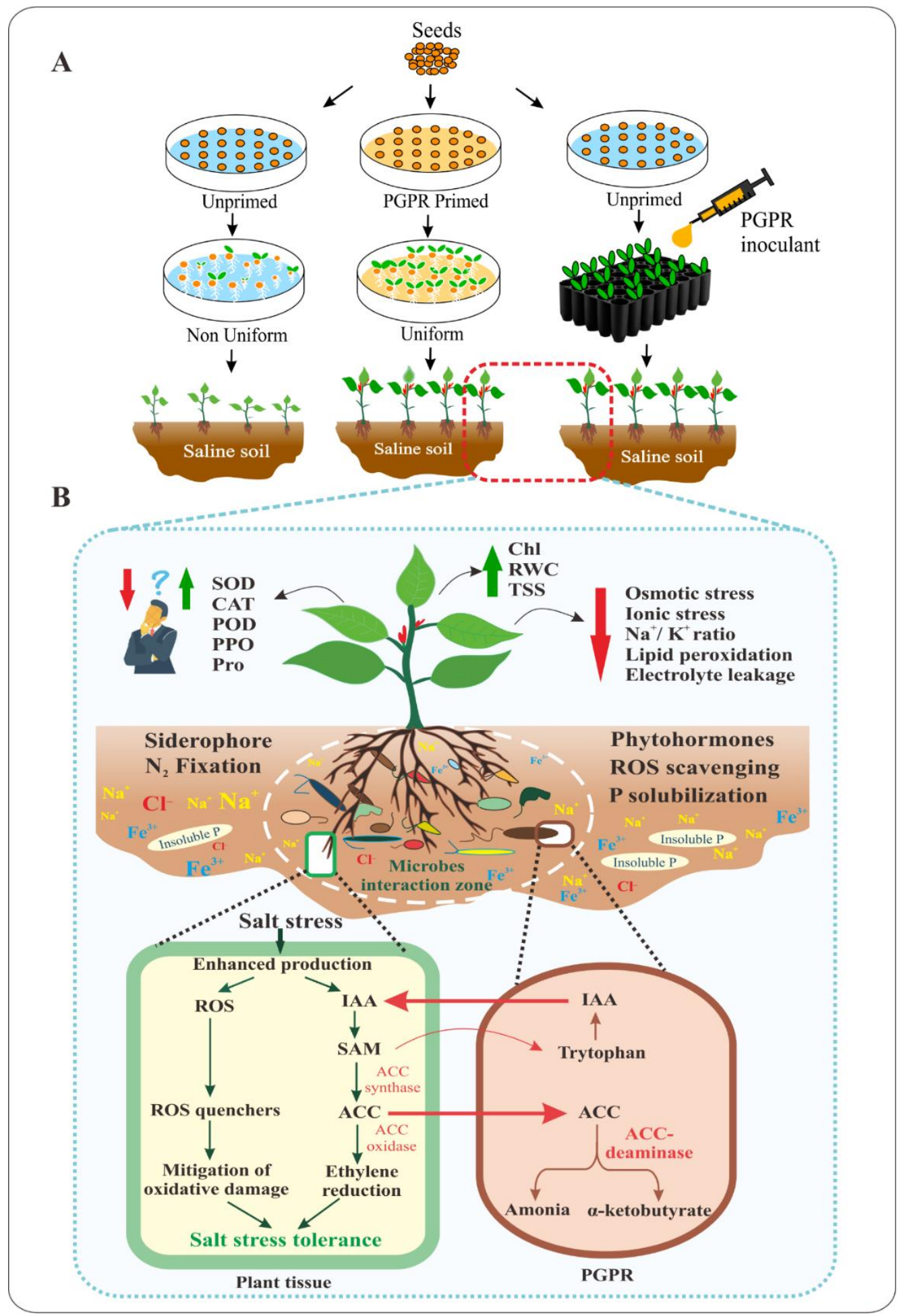

Figure 1. Roles of PGPR in alleviating salinity stress in plants. (A) represents the application of PGPR as microbial beneficial tools in seed biopriming technique and as green bioinoculants in seedlings treatment. The primed seeds demonstrate rapid germination and robust, uniform seedlings. (B) shows positive effects of PGPR on vegetative parameters and physio-biochemical indexes in PGPR-inoculated plants via various mechanisms e.g., production of OS, AEs to reduce osmotic and ionic stress, and EPS suppress toxic ions uptake and ion exposure. The fluctuation of AEs and OS profiles in PGPR-treated plants is also displayed in the left panel. The middle panel demonstrates key characteristics of PGPR including the production of Sid, phytohormones, EPS, N fixation and P solubilization. The lower panel emphasizes the importance of ACC deaminase-producing PGPR in ameliorating the inhibitory effects of excess ethylene on plant growth. 


\section{Roles of Multi-Omics Techniques in Deciphering Plant-Microbe Interactions}

The modes of action of PGPR on plant salt-stress response mechanism are diverse and complex and remain largely unclear, especially at the molecular level. In the study of Kim et al. (2014) [96], the colonization of Enterobacter sp. EJ01 in Arabidopsis root tissues conferred salt stress resistance by inducing salt stress responsive signaling pathways. Specifically, after EJ01 inoculation, the expression levels of DREB2b, RD29A, and RAB18 genes related to ABA-dependent and ABA-independent pathways were upregulated, even in the absence of salinity. The expression pattern of $R D 29 B$, however, was dependent on salt treatment. In addition, P5CS1, a Pro biosynthesis-related gene, was upregulated under salinity conditions. The inoculation of EJ01 into Arabidopsis plants induced the host basal innate immunity, as well as the rapid defense responses at systemic level so called induced systemic resistance (ISR). PGPR-elicited ISR was previously observed in Arabidopsis seedlings treated with VOCs from Bacillus subtilis GB03 and Bacillus amyloliquefaciens IN937a. The use of transgenic and mutant lines of Arabidopsis indicated that the ISR activated by the VOCs from GB03 was based on ethylene-dependent signaling pathway, whereas the ISR was triggered by VOCs from IN937a through an ethylene-independent signaling pathway [161]. In the study of Chen et al. [162], the upregulation of $\mathrm{Na}^{+} / \mathrm{H}^{+}$ antiporter (NHX) and $\mathrm{H}^{+}$-PPase genes in Bacillus amyloliquefaciens SQR9-inoculated maize shoots facilitated $\mathrm{Na}^{+}$sequestration into vacuoles. The recirculating of $\mathrm{Na}^{+}$from shoot to root via an elevated expression of high-affinity $\mathrm{K}^{+}$transporter 1 (HKT1) gene was observed in the treated maize plants. However, contrary to the results of Chen et al. [162], the lowest expression pattern of HKT1 gene was found in the Pseudomonas simiae + sodium nitroprusside (SNP) treated soybean plants [147]. A stable photosynthetic activity was maintained by the highly expressed RBCS (RubisCo small subunit), $R B C L$ (Rubisco large subunit) genes [162], similar to the upregulation of the Rubisco-encoding gene $r b c L$ in the Klebsiella-treated oat seedlings [44]. Moreover, the senescence rate in SQR9-inoculated treated maize was properly controlled by the reduced expression of NCED, a key gene in ABA synthesis pathway.

In all treatments, the expression levels of AEs-encoding genes $P O D$ and $C A T$ were the highest in the P. simiae + SNP-treated soybean plants [147]. Genes associated with Aux, CK, and GA signaling pathways in the Paenibacillus polymyxa YC0136-treated tobacco plants (Nicotiana tabacum L.) were found to be upregulated relative to the uninoculated control plants, along with the elevated expression of WRKY and MYB transcription factors (TFs) [163]. The WRKY and MYB TFs are responsible for gene regulations and have a great influence in every aspect of plant growth and development, as well as in plant stress responses $[164,165]$. Changes in the expression pattern of WRKY TF gene under salinity conditions were reported in previous studies [166-168]. These findings were in line with the transcriptome profiles of the rice roots bacterized with Azospirillum brasilense, in which the hormones-encoding genes (e.g., Aux efflux carriers, Aux-responsive genes, Aux response factors, ACC oxidase genes, ethylene insensitive 2, cytokinin-Oglucosyltransferases, and cytokinin dehydrogenase precursors) were significantly upregulated, as well as the major plant TFs families, namely, AP2/ERF family, MYB family, WRKY family, and the GRAS family [169]. The enhanced expressions of MYB and WRKY TFs were also noticed in the Dietzia natronolimnaea-inoculated wheat plants under salinity stress [152]. Furthermore, 9 genes in the SA pathway and 6 genes encoding phenylalanine ammonia lyase (PAL), a key enzyme in the phenylpropanoids metabolic pathway, were upregulated with respect to the control plants, resulting the induction of systemic resistance in tobacco host plant [163]. Malviya et al. [170] also found that the infection of Burkholderia anthina MYSP113 into the sugarcane plantlets cv. GXB-9 induced the upregulation of phenylpropanoid biosynthesis genes and amino acid biosynthesis pathways, in accordance with the findings from Liu et al. [163]. Likewise, phenylpropanoid biosynthesis was the most enriched pathway in both differentially expressed genes (DEGs) and abundant metabolites (DAMs) among all salt stress responses in barley rootzones [171]. In the Arthrobacter nitroguajacolicus-inoculated wheat roots treated with $200 \mathrm{mM} \mathrm{NaCl}$, 
8 genes responsible for Fe uptake, and 2 phosphatase-encoding genes were upregulated, as well as the upregulation of several transporter genes which were in charge of ions, sugars, oligopeptide, and amino acids transports [172].

On the one hand, PGPR influence the expression patterns in the host plants. On the other hand, changes in their transcriptome in response to their colonized plants were also recorded [173]. During the interaction with the host plant, 2 genes ilvB and PPYC1_23850 related to Aux biosynthesis, 3 genes belonging to cell motility category (e.g., fliG, $f l i H$, fliF), 31 genes related to transport proteins including 16 genes belong to ATP-binding cassette $(\mathrm{ABC})$, and 3 genes associated with a major facilitator superfamily (MFS) in P. polymyxa YC0136 were significantly upregulated [163]. It was thought that root exudates from tobacco attracted P. polymyxa YC0136 and may play roles as nutrients source for the growth of YC0136 strain. This explained the upregulation of numerous transport and cell motility genes in the bacterium. In response to host rice seedlings, Bacillus subtilis OKB105 also altered its transcriptomic patterns, in which 52 genes related to nutrients transport and metabolism were upregulated, suggesting the bacterium used carbohydrates and amino acids secreted by rice seedlings as carbon and energy sources. In contrast to the data in [163], many genes involved in chemotaxis and motility, however, were downregulated [173].

In summary, plants and PGPR influence each other in a mutualistic relationship. Regarding plant resistance to salinity, the microbes regulate the WRKY and MYB TFs which are widely distributed in higher plants. Subsequently, these master regulators will regulate the expression of their key downstream stress responsive genes. The plants, in turn, provide nutrients via root exudates for the growth of the microbes. This interaction benefits plants in non-stress conditions, and also in environmental challenging conditions.

\section{Promise, Limitations, and Future Directions}

Considerable PGPB-related studies that have been carried out in the last decades help to improve our knowledge concerning advantageous characteristics of PGPB, in both basic and applied aspects. However, most studies focused on estimating the parameters in vegetative growth stage, but rarely on evaluating the parameters that are related to reproductive stage such as GW and FW, numbers of flower, numbers of seed, fruit per plant, and plant yield. We found a scarcity of studies that evaluated beneficial effects of PGPB on attenuating yield loss and on improving nutrient values. In our opinion, this could be one of the main drawbacks of PGPB-related studies thus far if we consider the improvement of crop yields, productivity, and the quality of fruit/grain under high saline conditions to be our main goal in plant agriculture studies. In addition, in some studies, the lack of important measurements regarding ion contents, ROS levels, phytohormone concentrations, and electrolyte leakage in many studies make them difficult to evaluate the overall effects of PGPB on plants. Furthermore, the short exposure of plants to salinity in some studies unlikely reflects the real situation in fields where a variety of biotic and abiotic stresses endures simultaneously and lasts permanently.

The recognition of PGPR as safe, efficient, and appropriate bioinoculants for agricultural practice is widely accorded. However, the primary mechanisms employed by PGPR to promote plant defense against salt stress need to be deeply unraveled, especially changes in both Omics profiles (e.g., proteomics, transcriptomics, and metabolomics) in the treated plants and in the microbes during interaction with their hosts. In addition, the highly genetic variations of plants and PGPR are useful traits in coping with diverse environmental issues. However, this attribute also makes the reproducibility from previous studies' findings challenging. As discussed in the present review, the patterns of ROS quencher in PGPR-treated plants exhibited great differences from case to case mainly due to the genetic diversity. The synergistic and/or antagonistic effects between PGPR in consortia on plant growth and defense system, which occur commonly in terrestrial soil ecosystems, should also be thoroughly deciphered. Consequently, an integration of Omics technologies and systems biology should be considered in future studies to provide 
broader picture and more detailed information concerning plant-microbe interactions in a more complex scenario.

Author Contributions: Conceptualization, D.M.H.-T. and T.T.M.N.; Visualization, T.T.M.N.; WritingOriginal draft preparation, D.M.H.-T., T.T.M.N., E.H., S.-H.H., and C.-C.H.; Writing-Revised manuscript D.M.H.-T.; Supervision, C.-C.H. All authors have read and agreed to the published version of the manuscript.

Funding: This work received no external funding.

Institutional Review Board Statement: Not applicable.

Informed Consent Statement: Not applicable.

Data Availability Statement: Not applicable.

Conflicts of Interest: The authors declare no conflict of interest.

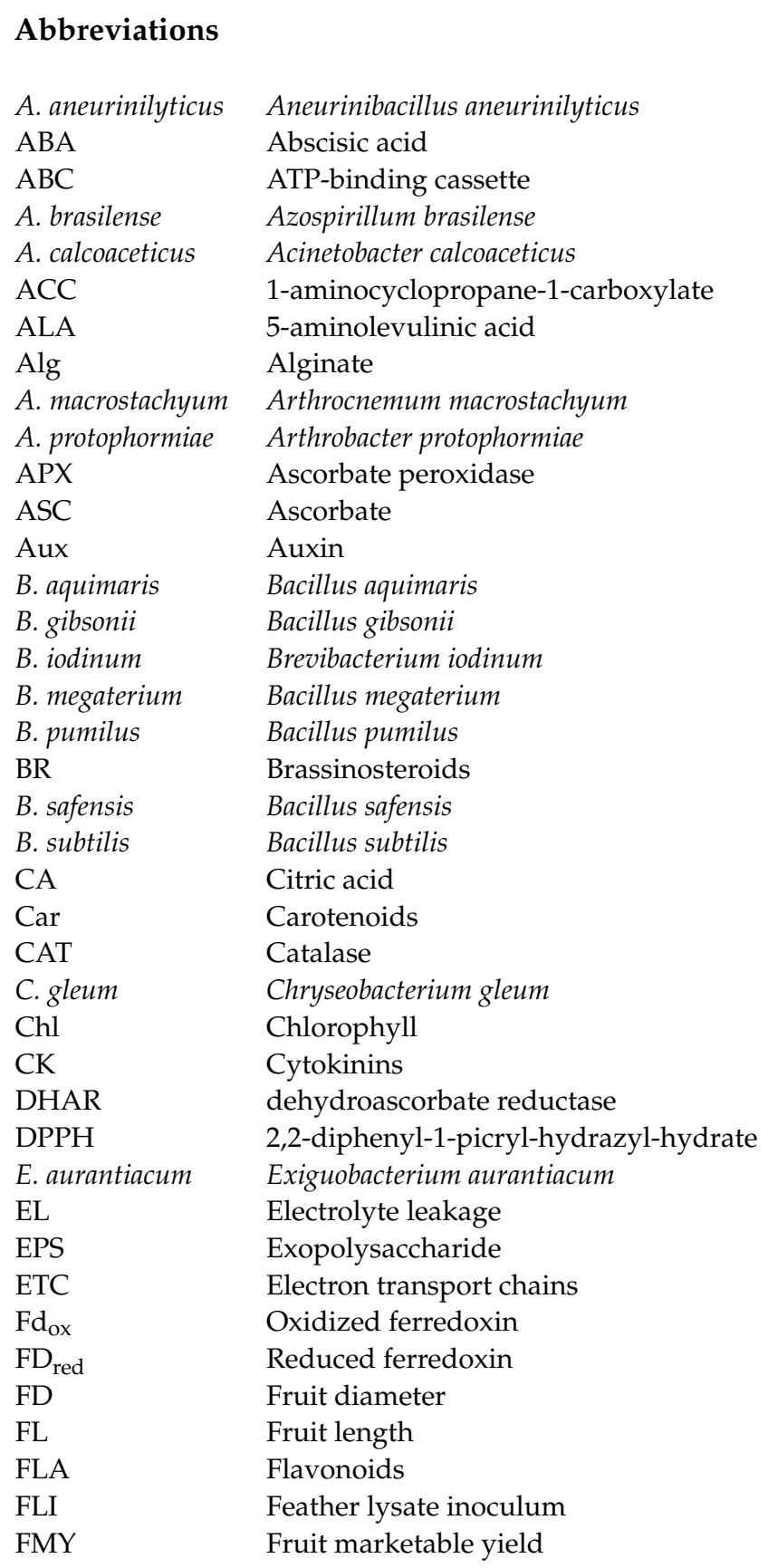




\begin{tabular}{|c|c|}
\hline FW & Fruit weight \\
\hline FY & Fruit yield \\
\hline GA & Gibberellins \\
\hline GLVs & Green leaf volatiles \\
\hline GP & Germination percentage \\
\hline G-POD & Guaiacol peroxidase \\
\hline GPr & Grain protein \\
\hline GR & Glutathione reductase \\
\hline GRA & Germination rate \\
\hline gs & Stomatal conductance \\
\hline GSH & Glutathione \\
\hline GST & Glutathione-S-transferase \\
\hline GW & Grain weight \\
\hline GY & Grain yield \\
\hline $\mathrm{H}_{2} \mathrm{O}_{2}$ & Hydrogen peroxide \\
\hline IAA & Indole-3-acetic acid \\
\hline ISR & Induced systemic resistance \\
\hline JA & Jasmonates \\
\hline K & Potassium \\
\hline K. sacchari & Kosakonia sacchari \\
\hline L. adecarboxylata & Leclercia adecarboxylata \\
\hline LP & Lipid peroxidation \\
\hline MA & Malic acid \\
\hline MDA & Malondialdehyde \\
\hline MDAR & monodehydroascorbate reductase \\
\hline Mel & Melatonin \\
\hline MeSA & Methyl salicylate \\
\hline MFS & Major facilitator superfamily \\
\hline M. oleivorans & Microbacterium oleivorans \\
\hline $\mathrm{N}$ & Nitrogen \\
\hline $\mathrm{N} / \mathrm{A}$ & Not available \\
\hline NF & Number of fruits per plant \\
\hline NL & Number of leaves per plant \\
\hline NT & Number of tillers per plant \\
\hline $\mathrm{O}_{2}^{\bullet-}$ & Superoxide radical \\
\hline OS & Osmolytes \\
\hline $\mathrm{P}$ & Phosphate \\
\hline PAs & Polyamines \\
\hline P. agglomerans & Pantoea agglomerans \\
\hline PAL & Phenylalanine ammonia lyase \\
\hline$P$. argentinensis & Pseudomonas argentinensis \\
\hline P. azotoformans & Pseudomonas azotoformans \\
\hline P. putida & Pseudomonas putida \\
\hline PCs & Phenolic compounds \\
\hline P. fluorescence & Pseudomonas fluorescence \\
\hline PGPR & Plant growth- promoting rhizobacteria \\
\hline $\mathrm{PH}$ & Plant height \\
\hline PHE & Phenols \\
\hline PhoPs & Photosynthetic pigments \\
\hline Pn & Net photosynthetic rate \\
\hline POD & Peroxidase \\
\hline PPs & Polyphenols \\
\hline $\mathrm{PPO}$ & Polyphenol oxidase \\
\hline P. putida & Pseudomonas putida \\
\hline Pro & Proline \\
\hline PSI & Photosystem I \\
\hline
\end{tabular}




$\begin{array}{ll}\text { P. yonginensis } & \text { Paenibacillus yonginensis } \\ \text { RDW } & \text { Root dry weight } \\ \text { RFW } & \text { Root fresh weight } \\ \text { RL } & \text { Root length } \\ \text { R. massiliae } & \text { Rhizobium massiliae } \\ \text { ROS } & \text { Reactive oxygen species } \\ \text { RWC } & \text { Relative water content } \\ \text { SA } & \text { Salicylic acid } \\ \text { SAM } & \text { S-adenosyl-L-methionine } \\ \text { SDM } & \text { Water-soluble dry matter } \\ \text { SDW } & \text { Shoot dry weight } \\ \text { SFW } & \text { Shoot fresh weight } \\ \text { Sid } & \text { Siderophore } \\ \text { SL } & \text { Shoot length } \\ \text { S. maltophilia } & \text { Stenotrophomonas maltophilia } \\ \text { SNP } & \text { Sodium nitroprusside } \\ \text { SOD } & \text { Superoxide dismutase } \\ \text { SpDW } & \text { Spike dry weight } \\ \text { StDW } & \text { Stem dry weight } \\ \text { StFW } & \text { Stem fresh weight } \\ \text { SVI } & \text { Seedling vigor index } \\ \text { SW } & \text { Seed weight } \\ \text { SY } & \text { Seed yield } \\ \text { Tchl } & \text { Total chlorophyll } \\ \text { TCP } & \text { Tocopherol } \\ \text { TFs } & \text { Transcription factors } \\ \text { TLA } & \text { Total leaves area per plant } \\ \text { TPDW } & \text { Translant dry weight } \\ \text { TPFW } & \text { Trehalose } \\ \text { Tr } & \text { Xanthobacter autotrophicus } \\ \text { Tre } & \text { Zield } \\ \text { TSS } & \text { Zhihengliuella halotolerans } \\ \text { X. autotrophicus } \\ \text { Y } & \\ \text { Z.halotolerans } & \end{array}$

\section{References}

1. de Lima-Neto, A.; Cavalcante, L.; Mesquita, F.d.O.; Souto, A.d.L.; dos Santos, G.; dos Santos, J.; de Mesquita, E. Papaya seedlings irrigation with saline water in soil with bovine biofertilizer. Chil. J. Agric. Res. 2016, 76, 236-242. [CrossRef]

2. Reints, J.; Dinar, A.; Crowley, D. Dealing with Water Scarcity and Salinity: Adoption of Water Efficient Technologies and Management Practices by California Avocado Growers. Sustainability 2020, 12, 3555. [CrossRef]

3. Tri, D.Q.; Tuyet, Q.T.T. Effect of Climate Change on the Salinity Intrusion: Case Study Ca River Basin, Vietnam. J. Clim. Chang. 2016, 2, 91-101. [CrossRef]

4. Gondek, M.; Weindorf, D.; Thiel, C.; Kleinheinz, G. Soluble Salts in Compost and Their Effects on Soil and Plants: A Review. Compost Sci. Util. 2020, 28, 59-75. [CrossRef]

5. $\quad$ Rasool, S.; Hameed, A.; Azooz, M.; Muneeb-u-Rehman; Siddiqi, T.; Ahmad, P. Salt Stress: Causes, Types and Responses of Plants. In Ecophysiology and Responses of Plants under Salt Stress; Ahmad, P., Azooz, M., Prasad, M., Eds.; Springer: New York, NY, USA, 2013; ISBN 978-1-4614-4747-4.

6. Attia, H.; Alamer, K.; Ouhibi, C.; Oueslati, S.; Lachaâl, M. Interaction Between Salt Stress and Drought Stress on Some Physiological Parameters in Two Pea Cultivars. Int. J. Bot. 2020, 16. [CrossRef]

7. Maathuis, F.; Amtmann, A. $\mathrm{K}^{+}$Nutrition and $\mathrm{Na}^{+}$Toxicity: The Basis of Cellular $\mathrm{K}^{+} / \mathrm{Na}^{+}$Ratios. Ann. Bot. 1999, 84, 123-133. [CrossRef]

8. Bernstein, N.; Meiri, A. Root Growth of Avocado is More Sensitive to Salinity than Shoot Growth. J. Am. Soc. Hortic. Sci. 2004, 129, 188-192. [CrossRef]

9. Neves, G.; Marchiosi, R.; Ferrarese, M.; Siqueira-Soares, R.; Ferrarese-Filho, O. Root Growth Inhibition and Lignification Induced by Salt Stress in Soybean. J. Agron. Crop Sci. 2010, 196, 467-473. [CrossRef]

10. Kafi, M.; Rahimi, Z. Effect of salinity and silicon on root characteristics, growth, water status, proline content and ion accumulation of purslane (Portulaca oleracea L.). Soil Sci. Plant Nutr. 2011, 57. [CrossRef] 
11. Fita, A.; Rodríguez-Burruezo, A.; Boscaiu, M.; Prohens, J.; Vicente, O. Breeding and Domesticating Crops Adapted to Drought and Salinity: A New Paradigm for Increasing Food Production. Front. Plant Sci. 2015, 6. [CrossRef]

12. Wang, W.; Vinocur, B.; Altman, A. Plant responses to drought, salinity and extreme temperatures: Towards genetic engineering for stress tolerance. Planta 2003, 218, 1-14. [CrossRef]

13. Jha, S. Chapter 14: Transgenic Approaches for Enhancement of Salinity Stress Tolerance in Plants. In Molecular Approaches in Plant Biology and Environmental Challenges; Singh, S., Upadhyay, S., Pandey, A., Kumar, S., Eds.; Energy, Environment and Sustainability; Springer Nature Singapore Pte Ltd.: Singapore, 2019; ISBN 978-981-15-0690-1.

14. Kaya, C.; Kirnak, H.; Higgs, D.; Saltali, K. Supplementary calcium enhances plant growth and fruit yield in strawberry cultivars grown at high $(\mathrm{NaCl})$ salinity. Sci. Hortic. 2002, 93, 65-74. [CrossRef]

15. Alzahrani, Y.; Kuşvuran, A.; Alharby, H.; Kuşvuran, S.; Rady, M. The defensive role of silicon in wheat against stress conditions induced by drought, salinity or cadmium. Ecotoxicol. Environ. Saf. 2018, 154, 187-196. [CrossRef]

16. Hassanvand, F.; Nejad, A.; Fanourakis, D. Morphological and physiological components mediating the silicon-induced enhancement of geranium essential oil yield under saline conditions. Ind. Crops Prod. 2019, 134, 19-25. [CrossRef]

17. Tian, S.; Guo, R.; Zou, X.; Zhang, X.; Yu, X.; Zhan, Y.; Ci, D.; Wang, M.; Wang, Y.; Si, T. Priming With the Green Leaf Volatile (Z)-3-Hexeny-1-yl Acetate Enhances Salinity Stress Tolerance in Peanut (Arachis hypogaea L.) Seedlings. Front. Plant Sci. 2019, 10. [CrossRef] [PubMed]

18. Yan, Z.-G.; Wang, C.-Z. Wound-induced green leaf volatiles cause the release of acetylated derivatives and a terpenoid in maize. Phytochemistry 2006, 67, 34-42. [CrossRef] [PubMed]

19. Wu, Y.; Liao, W.; Dawuda, M.; Hu, L.; Yu, J. 5-Aminolevulinic acid (ALA) biosynthetic and metabolic pathways and its role in higher plants: A review. Plant Growth Regul. 2019, 87, 357-374. [CrossRef]

20. Wu, Y.; Jin, X.; Liao, W.; Hu, L.; Dawuda, M.; Zhao, X.; Tang, Z.; Gong, T.; Yu, J. 5-Aminolevulinic Acid (ALA) Alleviated Salinity Stress in Cucumber Seedlings by Enhancing Chlorophyll Synthesis Pathway. Front. Plant Sci. 2018, 9, 635. [CrossRef]

21. Nawaz, K.; Chaudhary, R.; Sarwar, A.; Ahmad, B.; Gul, A.; Hano, C.; Abbasi, B.; Anjum, S. Melatonin as Master Regulator in Plant Growth, Development and Stress Alleviator for Sustainable Agricultural Production: Current Status and Future Perspectives. Sustainability 2021, 13, 294. [CrossRef]

22. Ke, Q.; Ye, J.; Wang, B.; Ren, J.; Yin, L.; Deng, X.; Wang, S. Melatonin Mitigates Salt Stress in Wheat Seedlings by Modulating Polyamine Metabolism. Front. Plant Sci. 2018, 9, 914. [CrossRef]

23. Liu, J.; Shabala, S.; Zhang, J.; Ma, G.; Chen, D.; Shabala, L.; Zeng, F.; Chen, Z.-H.; Zhou, M.; Venkataraman, G.; et al. Melatonin improves rice salinity stress tolerance by NADPH oxidase-dependent control of the plasma membrane $\mathrm{K}+$ transporters and $\mathrm{K}+$ homeostasis. Plant Cell Environ. 2020, 43, 2591-2605. [CrossRef]

24. Abd El-Azeem, S.; Elwan, M.; Sung, J.-K.; Ok, Y. Alleviation of Salt Stress in Eggplant (Solanum melongena L.) by Plant-GrowthPromoting Rhizobacteria. Commun. Soil Sci. Plant Anal. 2012, 43, 1303-1315. [CrossRef]

25. Hanin, M.; Ebel, C.; Ngom, M.; Laplaze, L.; Masmoudi, K. New Insights on Plant Salt Tolerance Mechanisms and Their Potential Use for Breeding. Front. Plant Sci. 2016, 7, 1-17. [CrossRef]

26. Santos, A.; da Silveira, J.; Bonifacio, A.; Rodrigues, A.; Figueiredo, M. Antioxidant response of cowpea co-inoculated with plant growth-promoting bacteria under salt stress. Braz. J. Microbiol. 2018, 49, 513-521. [CrossRef] [PubMed]

27. Gill, S.; Tuteja, N. Reactive oxygen species and antioxidant machinery in abiotic stress tolerance in crop plants. Plant Physiol. Biochem. 2010, 48, 909-930. [CrossRef] [PubMed]

28. Jogawat, A. Chapter 5: Osmolytes and their Role in Abiotic Stress Tolerance in Plants. In Molecular Plant Abiotic Stress: Biology and Biotechnology; Roychoudhury, A., Tripathi, D., Eds.; John Wiley \& Sons Ltd.: Hoboken, NJ, USA, 2019; pp. 91-104. ISBN 978-1-11946366-5.

29. Tester, M.; Davenport, R. Na+tolerance and Na+ transport in higher plants. Ann. Bot. 2003, 91, 503-527. [CrossRef] [PubMed]

30. Cheng, Z.; Woody, O.; McConkey, B.; Glick, B. Combined effects of the plant growth-promoting bacterium Pseudomonas putida UW4 and salinity stress on the Brassica napus proteome. Appl. Soil Ecol. 2012, 61, 255-263. [CrossRef]

31. Shahid, M.; Sarkhosh, A.; Khan, N.; Balal, R.; Ali, S.; Rossi, L.; Gómez, C.; Mattson, N.; Nasim, W.; Garcia-Sanchez, F. Insights into the Physiological and Biochemical Impacts of Salt Stress on Plant Growth and Development. Agronomy 2020, 10, 938. [CrossRef]

32. Manishankar, P.; Wang, N.; Köster, P.; Alatar, A.; Kudla, J. Calcium signaling during salt stress and in the regulation of ion homeostasis. J. Exp. Bot. 2018, 69, 4215-4226. [CrossRef] [PubMed]

33. Zhang, M.; Fang, Y.; Ji, Y.; Jiang, Z.; Wang, L. Effects of salt stress on ion content, antioxidant enzymes and protein profile in different tissues of Broussonetia papyrifera. S. Afr. J. Bot. 2013, 85, 1-9. [CrossRef]

34. Taïbi, K.; Taïbi, F.; Abderrahim, L.; Ennajah, A.; Belkhodja, M.; Mulet, J. Effect of salt stress on growth, chlorophyll content, lipid peroxidation and antioxidant defence systems in Phaseolus vulgaris L. S. Afr. J. Bot. 2016, 105, 306-312. [CrossRef]

35. Abd_Allah, E.; Alqarawi, A.; Hashem, A.; Radhakrishnan, R.; Al-Huqail, A.; Al-Otibi, F.; Malik, J.; Alharbi, R.; Egamberdieva, D. Endophytic bacterium Bacillus subtilis (BERA 71) improves salt tolerance in chickpea plants by regulating the plant defense mechanisms. J. Plant Interact. 2018, 13, 37-44. [CrossRef]

36. Shahid, M.; Ameen, F.; Maheshwari, H.; Ahmed, B.; AlNadhari, S.; Khan, M. Colonization of Vigna radiata by a halotolerant bacterium Kosakonia sacchari improves the ionic balance, stressor metabolites, antioxidant status and yield under $\mathrm{NaCl}$ stress. Appl. Soil Ecol. 2021, 158, 103809. [CrossRef] 
37. Magán, J.; Gallardo, M.; Thompson, R.; Lorenzo, P. Effects of salinity on fruit yield and quality of tomato grown in soil-less culture in greenhouses in Mediterranean climatic conditions. Agric. Water Manag. 2008, 95, 1041-1055. [CrossRef]

38. Navarro, J.; Garrido, C.; Flores, P.; Martínez, V. The effect of salinity on yield and fruit quality of pepper grown in perlite. Span. J. Agric. Res. 2010, 8, 142-150. [CrossRef]

39. Wungrampha, S.; Joshi, R.; Singla-Pareek, S.; Pareek, A. Photosynthesis and salinity: Are these mutually exclusive? Photosynthetica 2018, 56, 366-381. [CrossRef]

40. Khan, A.; Sirajuddin;; Zhao, X.; Javed, M.; Khan, K.; Bano, A.; Shen, R.; Masood, S. Bacillus pumilus enhances tolerance in rice (Oryza sativa L.) to combined stresses of $\mathrm{NaCl}$ and high boron due to limited uptake of $\mathrm{Na}^{+}$. Environ. Exp. Bot. 2016, 124, 120-129. [CrossRef]

41. Kang, S.-M.; Radhakrishnan, R.; Khan, A.; Kim, M.-J.; Park, J.-M.; Kim, B.-R.; Shin, D.-H.; Lee, I.-J. Gibberellin secreting rhizobacterium, Pseudomonas putida H-2-3 modulates the hormonal and stress physiology of soybean to improve the plant growth under saline and drought conditions. Plant Physiol. Biochem. 2014, 84, 115-124. [CrossRef]

42. Li, H.; Jiang, X. Inoculation with plant growth-promoting bacteria (PGPB) improves salt tolerance of maize seedling. Russ. J. Plant Physiol. 2017, 64, 235-241. [CrossRef]

43. Kang, S.-M.; Khan, A.; Waqas, M.; You, Y.-H.; Kim, J.-H.; Kim, J.-G.; Hamayun, M.; Lee, I.-J. Plant growth-promoting rhizobacteria reduce adverse effects of salinity and osmotic stress by regulating phytohormones and antioxidants in Cucumis sativus. J. Plant Interact. 2014, 9, 673-682. [CrossRef]

44. Sapre, S.; Gontia-Mishra, I.; Tiwari, S. Klebsiella sp. confers enhanced tolerance to salinity and plant growth promotion in oat seedlings (Avena sativa). Microbiol. Res. 2018, 206, 25-32. [CrossRef]

45. Akram, W.; Aslam, H.; Ahmad, S.; Anjum, T.; Yasin, N.; Khan, W.; Ahmad, A.; Guo, J.; Wu, T.; Luo, W.; et al. Bacillus megaterium strain A12 ameliorates salinity stress in tomato plants through multiple mechanisms. J. Plant Interact. 2019, 14, 506-518. [CrossRef]

46. Orozco-Mosqueda, M.; Duan, J.; DiBernardo, M.; Zetter, E.; Campos-García, J.; Glick, B.; Santoyo, G. The Production of ACC Deaminase and Trehalose by the Plant Growth Promoting Bacterium Pseudomonas sp. UW4 Synergistically Protect Tomato Plants Against Salt Stress. Front. Microbiol. 2019, 10, 1392. [CrossRef] [PubMed]

47. Vaishnav, A.; Singh, J.; Singh, P.; Rajput, R.; Singh, H.; Sarma, B. Sphingobacterium sp. BHU-AV3 Induces Salt Tolerance in Tomato by Enhancing Antioxidant Activities and Energy Metabolism. Front. Microbiol. 2020, 11. [CrossRef] [PubMed]

48. Alexander, A.; Singh, V.; Mishra, A. Halotolerant PGPR Stenotrophomonas maltophilia BJ01 Induces Salt Tolerance by Modulating Physiology and Biochemical Activities of Arachis hypogaea. Front. Microbiol. 2020, 11, 8289. [CrossRef]

49. Sarkar, A.; Ghosh, P.; Pramanik, K.; Mitra, S.; Soren, T.; Pandey, S.; Mondal, M.; Maiti, T. A halotolerant Enterobacter sp. displaying ACC deaminase activity promotes rice seedling growth under salt stress. Res. Microbiol. 2018, 169, 20-32. [CrossRef]

50. Sukweenadhi, J.; Balusamy, S.; Kim, Y.-J.; Lee, C.; Kim, Y.-J.; Koh, S.; Yang, D. A Growth-Promoting Bacteria, Paenibacillus yonginensis DCY84T Enhanced Salt Stress Tolerance by Activating Defense-Related Systems in Panax ginseng. Front. Plant Sci. 2018, 9, 813. [CrossRef]

51. Ansari, F.; Ahmad, I.; Pichtel, J. Growth stimulation and alleviation of salinity stress to wheat by the biofilm forming Bacillus pumilus strain FAB10. Appl. Soil Ecol. 2019, 143, 45-54. [CrossRef]

52. Hamann, T. The Plant Cell Wall Integrity Maintenance Mechanism-Concepts for Organization and Mode of Action. Plant Cell Physiol. 2015, 56, 215-223. [CrossRef]

53. Yin, H.; Xu, L.; Porter, N. Free Radical Lipid Peroxidation: Mechanisms and Analysis. Chem. Rev. 2011, 111, 5944-5972. [CrossRef]

54. Corpas, F.; Gupta, D.; Palma, J. Chapter 1: Production Sites of Reactive Oxygen Species (ROS) in Organelles from Plant Cells. In Reactive Oxygen Species and Oxidative Damage in Plants; Gupta, D., Palma, J., Corpas, F., Eds.; Springer International Publishing: Cham, Switzerland, 2015; ISBN 978-3-319-20421-5.

55. Gutteridge, J. Lipid Peroxidation and Antioxidants as Biomarkers of Tissue Damage. Clin. Chem. 1995, 41, 1819-1828. [CrossRef] [PubMed]

56. Awasthi, J.; Saha, B.; Chowardhara, B.; Devi, S.; Borgohain, P.; Panda, S. Qualitative Analysis of Lipid Peroxidation in Plants under Multiple Stress Through Schiff's Reagent: A Histochemical Approach. Bio-Protocol 2018, 8, e2807. [CrossRef]

57. Sarkar, A.; Pramanik, K.; Mitra, S.; Soren, T.; Maiti, T. Enhancement of growth and salt tolerance of rice seedlings by ACC deaminase-producing Burkholderia sp. MTCC 12259. J. Plant Physiol. 2018, 231, 434-442. [CrossRef]

58. Khalid, M.; Bilal, M.; Hassani, D.; Iqbal, H.; Wang, H.; Huang, D. Mitigation of salt stress in white clover (Trifolium repens) by Azospirillum brasilense and its inoculation effect. Bot. Stud. 2017, 58. [CrossRef] [PubMed]

59. Ishibashi, Y.; Kasa, S.; Sakamoto, M.; Aoki, N.; Kai, K.; Yuasa, T.; Hanada, A.; Yamaguchi, S.; Iwaya-Inoue, M. A Role for Reactive Oxygen Species Produced by NADPH Oxidases in the Embryo and Aleurone Cells in Barley Seed Germination. PLoS ONE 2015, 10, e143173. [CrossRef]

60. Tsukagoshi, H.; Busch, W.; Benfey, P. Transcriptional Regulation of ROS Controls Transition from Proliferation to Differentiation in the Root. Cell 2010, 143, 606-616. [CrossRef]

61. Tognetti, V.; Bielach, A.; Hrtyan, M. Redox regulation at the site of primary growth: Auxin, cytokinin and ROS crosstalk. Plant Cell Environ. 2017, 40, 2586-2605. [CrossRef]

62. Zeng, J.; Dong, Z.; Wu, H.; Tian, Z.; Zhao, Z. Redox regulation of plant stem cell fate. EMBO J. 2017, 36, 2844-2855. [CrossRef]

63. Aarti, P.; Tanaka, R.; Tanaka, A. Effects of oxidative stress on chlorophyll biosynthesis in cucumber (Cucumis sativus) cotyledons. Physiol. Plant. 2006, 128, 186-197. [CrossRef] 
64. Huang, H.; Ullah, F.; Zhou, D.-X.; Yi, M.; Zhao, Y. Mechanisms of ROS Regulation of Plant Development and Stress Responses. Front. Plant Sci. 2019, 10, 800. [CrossRef]

65. Abdelgawad, H.; Zinta, G.; Hegab, M.; Pandey, R.; Asard, H.; Abuelsoud, W. High Salinity Induces Different Oxidative Stress and Antioxidant Responses in Maize Seedlings Organs. Front. Plant Sci. 2016, 7, 276. [CrossRef]

66. Nimse, S.; Pal, D. Free radicals, natural antioxidants, and their reaction mechanisms. RSC Adv. 2015, 5, 27986-28006. [CrossRef]

67. Per, T.; Khan, N.; Reddy, P.; Masood, A.; Hasanuzzaman, M.; Khan, M.; Anjum, N. Approaches in modulating proline metabolism in plants for salt and drought stress tolerance: Phytohormones, mineral nutrients and transgenics. Plant Physiol. Biochem. 2017, 115, 126-140. [CrossRef] [PubMed]

68. Hayat, S.; Hayat, Q.; Alyemeni, M.; Wani, A.; Pichtel, J.; Ahmad, A. Role of proline under changing environments. Plant Signal. Behav. 2012, 7. [CrossRef] [PubMed]

69. Matysik, J.; Alia; Bhalu, B.; Mohanty, B. Molecular mechanisms of quenching of reactive oxygen species by proline under stress in plants. Curr. Sci. 2002, 82, 525-532.

70. Hossain, M.; Hoque, M.; Burritt, D.; Fujita, M. Chapter 16: Proline Protects Plants Against Abiotic Oxidative Stress: Biochemical and Molecular Mechanisms. In Oxidative Damage to Plants-Antioxidant Networks and Signaling; Ahmad, P., Ed.; Academic Press Inc.: Cambridge, MA, USA, 2014; pp. 477-522. ISBN 978-0-12-799963-0.

71. Iqbal, N.; Khan, N.; Ferrante, A.; Trivellini, A.; Francini, A.; Khan, M. Ethylene Role in Plant Growth, Development and Senescence: Interaction with Other Phytohormones. Front. Plant Sci. 2017, 8, 475. [CrossRef] [PubMed]

72. Boex-Fontvieille, E.; Rustgi, S.; von Wettstein, D.; Pollmann, S.; Reinbothe, S.; Reinbothe, C. An Ethylene-Protected Achilles' Heel of Etiolated Seedlings for Arthropod Deterrence. Front. Plant Sci. 2016, 7, 1246. [CrossRef] [PubMed]

73. Zapata, P.; Serrano, M.; García-Legaz, M.; Pretel, M.; Botella, M. Short Term Effect of Salt Shock on Ethylene and Polyamines Depends on Plant Salt Sensitivity. Front. Plant Sci. 2017, 8, 855. [CrossRef] [PubMed]

74. Arraes, F.; Beneventi, M.; de Sa, M.; Paixao, J.; Albuquerque, E.; Marin, S.; Purgatto, E.; Nepomuceno, A.; Grossi-de-Sa, M. Implications of ethylene biosynthesis and signaling in soybean drought stress tolerance. BMC Plant Biol. 2015, 15. [CrossRef]

75. Gamalero, E.; Glick, B. Bacterial Modulation of Plant Ethylene Levels. Plant Physiol. 2015, 169, 13-22. [CrossRef]

76. Tabassum, B.; Khan, A.; Tariq, M.; Ramzan, M.; Khan, M.; Shahid, N.; Aaliya, K. Bottlenecks in commercialisation and future prospects of PGPR. Appl. Soil Ecol. 2017, 121, 102-117. [CrossRef]

77. Ruiu, L. Plant-Growth-Promoting Bacteria (PGPB) against Insects and Other Agricultural Pests. Agronomy 2020, 10, 861. [CrossRef]

78. Saravanakumar, D.; Thomas, A.; Banwarie, N. Antagonistic potential of lipopeptide producing Bacillus amyloliquefaciens against major vegetable pathogens. Eur. J. Plant Pathol. 2019, 154, 319-335. [CrossRef]

79. Oteino, N.; Lally, R.; Kiwanuka, S.; Lloyd, A.; Ryan, D.; Germaine, K.; Dowling, D. Plant growth promotion induced by phosphate solubilizing endophytic Pseudomonas isolates. Front. Microbiol. 2015, 6, 1-9. [CrossRef] [PubMed]

80. Zahid, M.; Abbasi, M.; Hameed, S.; Rahim, N. Isolation and identification of indigenous plant growth promoting rhizobacteria from Himalayan region of Kashmir and their effect on improving growth and nutrient contents of maize (Zea mays L.). Front. Microbiol. 2015, 6, 207. [CrossRef] [PubMed]

81. Dimkpa, C.; Weinand, T.; Asch, F. Plant-rhizobacteria interactions alleviate abiotic stress conditions. Plant Cell Environ. 2009, 32, 1682-1694. [CrossRef]

82. Arora, N.; Fatima, T.; Mishra, J.; Mishra, I.; Verma, S.; Verma, R.; Verma, M.; Bhattacharya, A.; Verma, P.; Mishra, P.; et al. Halo-tolerant plant growth promoting rhizobacteria for improving productivity and remediation of saline soils. J. Adv. Res. 2020, 26, 69-82. [CrossRef]

83. Fouda, A.; Hassan, S.; Eid, A.; Ewais, E. The Interaction Between Plants and Bacterial Endophytes Under Salinity Stress. In Endophytes and Secondary Metabolites; Jha, S., Ed.; Reference Series in Phytochemistry; Springer: Cham, Switzerland, 2019; pp. 1-17. ISBN 978-3-319-76900-4.

84. Lata, R.; Chowdhury, S.; Gond, S.; White Jr, J. Induction of abiotic stress tolerance in plants by endophytic microbes. Lett. Appl. Microbiol. 2018, 66, 268-276. [CrossRef]

85. Ashraf, M.; Hasnain, S.; Berge, O.; Mahmood, T. Inoculating wheat seedlings with exopolysaccharide-producing bacteria restricts sodium uptake and stimulates plant growth under salt stress. Biol. Fertil. Soils 2004, 40, 157-162. [CrossRef]

86. Upadhyay, S.; Singh, J.; Singh, D. Exopolysaccharide-Producing Plant Growth-Promoting Rhizobacteria Under Salinity Condition. Pedosphere 2011, 21, 214-222. [CrossRef]

87. Atouei, M.; Pourbabaee, A.; Shorafa, M. Alleviation of Salinity Stress on Some Growth Parameters of Wheat by ExopolysaccharideProducing Bacteria. Iran. J. Sci. Technol. Trans. Sci. 2019, 43, 2725-2733. [CrossRef]

88. Gupta, S.; Pandey, S. ACC Deaminase Producing Bacteria With Multifarious Plant Growth Promoting Traits Alleviates Salinity Stress in French Bean (Phaseolus vulgaris) Plants. Front. Microbiol. 2019, 10, 1506. [CrossRef]

89. Glick, B.; Todorovic, B.; Czarny, J.; Cheng, Z.; Duan, J.; McConkey, B. Promotion of Plant Growth by Bacterial ACC Deaminase. Crit. Rev. Plant Sci. 2007, 26, 227-242. [CrossRef]

90. Pierik, R.; Tholen, D.; Poorter, H.; Visser, E.; Voesenek, L. The Janus face of ethylene: Growth inhibition and stimulation. Trends Plant Sci. 2006, 11, 176-183. [CrossRef]

91. Abeles, F.; Morgan, P.; Saltveit, M., Jr. Ethylene in Plant Biology, 2nd ed.; Academic Press Inc.: Cambridge, MA, USA, 1992; ISBN 978-0-08-091628-6. 
92. Stearns, J.; Glick, B. Transgenic plants with altered ethylene biosynthesis or perception. Biotechnol. Adv. 2003, 21, 193-210. [CrossRef]

93. Glick, B. Bacteria with ACC deaminase can promote plant growth and help to feed the world. Microbiol. Res. 2014, 69, 30-39. [CrossRef] [PubMed]

94. Fuertes-Mendizábal, T.; Bastías, E.; González-Murua, C.; González-Moro, M. Nitrogen Assimilation in the Highly Salt- and Boron-Tolerant Ecotype Zea mays L. Amylacea. Plants 2020, 9, 322. [CrossRef] [PubMed]

95. Barnawal, D.; Bharti, N.; Maji, D.; Chanotiya, C.; Kalra, A. ACC deaminase-containing Arthrobacter protophormiae induces $\mathrm{NaCl}$ stress tolerance through reduced ACC oxidase activity and ethylene production resulting in improved nodulation and mycorrhization in Pisum sativum. J. Plant Physiol. 2014, 171, 884-894. [CrossRef]

96. Kim, K.; Jang, Y.-J.; Lee, S.-M.; Oh, B.-T.; Chae, J.-C.; Lee, K.-J. Alleviation of Salt Stress by Enterobacter sp. EJ01 in Tomato and Arabidopsis Is Accompanied by Up-Regulation of Conserved Salinity Responsive Factors in Plants. Mol. Cells 2014, 37, 109-117. [CrossRef]

97. Habib, S.; Kausar, H.; Saud, H. Plant Growth-Promoting Rhizobacteria Enhance Salinity Stress Tolerance in Okra through ROS-Scavenging Enzymes. BioMed Res. Int. 2016, 2016. [CrossRef]

98. Orhan, F. Alleviation of salt stress by halotolerant and halophilic plant growth-promoting bacteria in wheat (Triticum aestivum). Braz. J. Microbiol. 2016, 47, 621-627. [CrossRef]

99. Bhise, K.; Bhagwat, P.; Dandge, P. Synergistic effect of Chryseobacterium gleum sp. SUK with ACC deaminase activity in alleviation of salt stress and plant growth promotion in Triticum aestivum L. 3 Biotech 2017, 7. [CrossRef] [PubMed]

100. Zerrouk, I.; Benchabane, M.; Khelifi, L.; Yokawa, K.; Ludwig-Müller, J.; Baluska, F. A Pseudomonas strain isolated from date-palm rhizospheres improves root growth and promotes root formation in maize exposed to salt and aluminum stress. J. Plant Physiol. 2016, 191, 111-119. [CrossRef]

101. Hahm, M.-S.; Son, J.-S.; Hwang, Y.-J.; Kwon, D.-K.; Ghim, S.-Y. Alleviation of Salt Stress in Pepper (Capsicum annum L.) Plants by Plant Growth-Promoting Rhizobacteria. J. Microbiol. Biotechnol. 2017, 27, 1790-1797. [CrossRef] [PubMed]

102. Singh, R.; Jha, P. The PGPR Stenotrophomonas maltophilia SBP-9 Augments Resistance against Biotic and Abiotic Stress in Wheat Plants. Front. Microbiol. 2017, 8, 1945. [CrossRef] [PubMed]

103. Cherif-Silini, H.; Thissera, B.; Bouket, A.; Saadaoui, N.; Silini, A.; Eshelli, M.; Alenezi, F.; Vallat, A.; Luptakova, L.; Yahiaoui, B.; et al. Durum Wheat Stress Tolerance Induced by Endophyte Pantoea agglomerans with Genes Contributing to Plant Functions and Secondary Metabolite Arsenal. Int. J. Mol. Sci. 2019, 20, 3989. [CrossRef] [PubMed]

104. Kang, S.-M.; Shahzad, R.; Bilal, S.; Khan, A.; Park, Y.-G.; Lee, K.-E.; Asaf, S.; Khan, M.; Lee, I.-J. Indole-3-acetic-acid and ACC deaminase producing Leclercia adecarboxylata MO1 improves Solanum lycopersicum L. growth and salinity stress tolerance by endogenous secondary metabolites regulation. BMC Microbiol. 2019, 19. [CrossRef] [PubMed]

105. Phour, M.; Sindhu, S. Amelioration of salinity stress and growth stimulation of mustard (Brassica juncea L.) by salt-tolerant Pseudomonas species. Appl. Soil Ecol. 2020, 149. [CrossRef]

106. Misra, S.; Chauhan, P. ACC deaminase-producing rhizosphere competent Bacillus spp. mitigate salt stress and promote Zea mays growth by modulating ethylene metabolism. 3 Biotech 2020, 10. [CrossRef]

107. Zhu, Z.; Zhang, H.; Leng, J.; Niu, H.; Chen, X.; Liu, D.; Chen, Y.; Ying, H. Isolation and characterization of plant growth-promoting rhizobacteria and their effects on the growth of Medicago sativa L. under salinity conditions. Antonie Leeuwenhoek 2020, 113, 1263-1278. [CrossRef]

108. Khan, A.; Waqas, M.; Asaf, S.; Kamran, M.; Shahzad, R.; Bilal, S.; Khan, M.; Kang, S.-M.; Kim, Y.-H.; Yun, B.-W.; et al. Plant growth-promoting endophyte Sphingomonas sp. LK11 alleviates salinity stress in Solanum pimpinellifolium. Environ. Exp. Bot. 2017, 133, 58-69. [CrossRef]

109. Ilyas, N.; Mazhar, R.; Yasmin, H.; Khan, W.; Iqbal, S.; El Enshasy, H.; Dailin, D. Rhizobacteria Isolated from Saline Soil Induce Systemic Tolerance in Wheat (Triticum aestivum L.) against Salinity Stress. Agronomy 2020, 10, 989. [CrossRef]

110. Mukherjee, P.; Mitra, A.; Roy, M. Halomonas Rhizobacteria of Avicennia marina of Indian Sundarbans Promote Rice Growth Under Saline and Heavy Metal Stresses Through Exopolysaccharide Production. Front. Microbiol. 2019, 10, 1207. [CrossRef] [PubMed]

111. Nadeem, S.; Zahir, Z.; Naveed, M.; Arshad, M.; Shahzad, S. Variation in growth and ion uptake of maize due to inoculation with plant growth promoting rhizobacteria under salt stress. Soil Environ. 2006, 25, 78-84.

112. Ahmed, A.; Hasnain, S. Auxins as One of the Factors of Plant Growth Improvement by Plant Growth Promoting Rhizobacteria Pol. J. Microbiol. 2014, 63, 261-266. [CrossRef] [PubMed]

113. Cordovez, V.; Schop, S.; Hordijk, K.; de Boulois, H.; Coppens, F.; Hanssen, I.; Raaijmakers, J.; Carrión, V. Priming of Plant Growth Promotion by Volatiles of Root-Associated Microbacterium spp. Appl. Environ. Microbiol. 2018, 84. [CrossRef] [PubMed]

114. Tahir, H.; Wu, H.; Raza, W.; Hanif, A.; Wu, L.; Colman, M.; Gao, X. Plant Growth Promotion by Volatile Organic Compounds Produced by Bacillus subtilis SYST2. Front. Microbiol. 2017, 8, 171. [CrossRef]

115. Saraf, M.; Jha, C.; Patel, D. The Role of ACC Deaminase Producing PGPR in Sustainable Agriculture. In Plant Growth and Health Promoting Bacteria; Maheshwari, D., Ed.; Microbiology Monographs; Springer: Berlin/Heidelberg, Germany, 2010; Volume 18, pp. 365-385. ISSN 1862-5584. 
116. López-Bucio, J.; Campos-Cuevas, J.; Hernández-Calderón, E.; Velásquez-Becerra, C.; Farías-Rodríguez, R.; Macías-Rodríguez, L.; Valencia-Cantero, E. Bacillus megaterium Rhizobacteria Promote Growth and Alter Root-System Architecture Through an Auxinand Ethylene-Independent Signaling Mechanism in Arabidopsis thaliana. MPMI 2007, 20, 207-217. [CrossRef] [PubMed]

117. Chu, T.N.; Bui, L.V.; Hoang, M.T.T. Pseudomonas PS01 Isolated from Maize Rhizosphere Alters Root System Architecture and Promotes Plant Growth. Microorganisms 2020, 8, 471. [CrossRef]

118. Liotti, R.; da Silva Figueiredo, M.; da Silva, G.; de Mendonçad, E.; Soares, M. Diversity of cultivable bacterial endophytes in Paullinia cupana and their potential for plant growth promotion and phytopathogen control. Microbiol. Res. 2018, 207, 8-18. [CrossRef]

119. Khare, E.; Mishra, J.; Arora, N. Multifaceted Interactions Between Endophytes and Plant: Developments and Prospects. Front. Microbiol. 2018, 9, 2732. [CrossRef] [PubMed]

120. Mena-Violante, H.; Olalde-Portugal, V. Alteration of tomato fruit quality by root inoculation with plant growth-promoting rhizobacteria (PGPR): Bacillus subtilis BEB-13bs. Sci. Hortic. 2007, 113, 103-106. [CrossRef]

121. Kumar, A.; Maurya, B.; Raghuwanshi, R. Isolation and characterization of PGPR and their effect on growth, yield and nutrient content in wheat (Triticum aestivum L.). Biocatal. Agric. Biotechnol. 2014, 3, 121-128. [CrossRef]

122. Nawaz, A.; Shahbaz, M.; Asadullah, A.I.; Marghoob, M.; Imtiaz, M.; Mubeen, F. Potential of Salt Tolerant PGPR in Growth and Yield Augmentation of Wheat (Triticum aestivum L.) Under Saline Conditions. Front. Microbiol. 2020, 11, 2019. [CrossRef]

123. Awad, N.; Turky, A.; Abdelhamid, M.; Attia, M. Ameliorate of environmental salt stress on the growth of Zea mays L. plants by exopolysaccharides producing bacteria. J. Appl. Sci. Res. 2012, 8, 2033-2044.

124. Abd El-Ghany, M.; Attia, M. Effect of Exopolysaccharide-Producing Bacteria and Melatonin on Faba Bean Production in Saline and Non-Saline Soil. Agronomy 2020, 10, 316. [CrossRef]

125. Tsao, R. Chemistry and Biochemistry of Dietary Polyphenols. Nutrients 2010, 2, 1231-1246. [CrossRef]

126. Hichem, H.; Mounir, D.; Naceur, E. Differential responses of two maize (Zea mays L.) varieties to salt stress: Changes on polyphenols composition of foliage and oxidative damages. Ind. Crops Prod. 2009, 30, 144-151. [CrossRef]

127. Ksouri, R.; Megdiche, W.; Debez, A.; Falleh, H.; Grignon, C.; Abdelly, C. Salinity effects on polyphenol content and antioxidant activities in leaves of the halophyte Cakile maritima. Plant Physiol. Biochem. 2007, 45, 244-249. [CrossRef] [PubMed]

128. Rojas-Tapias, D.; Moreno-Galván, A.; Pardo-Díaz, S.; Obando, M.; Rivera, D.; Bonilla, R. Effect of inoculation with plant growth-promoting bacteria (PGPB) on amelioration of saline stress in maize (Zea mays). Appl. Soil Ecol. 2012, 61, 264-272. [CrossRef]

129. Kasinath, B.; Senthivel, T.; Ganeshmurthy, A.; Nagegowda, N.; Kumar, M. Effect of Magnesium application on chlorophyll content and yield of tomato. Plant Arch. 2014, 14, 801-804.

130. Hermans, C.; Verbruggen, N. Physiological characterization of Mg deficiency in Arabidopsis thaliana. J. Exp. Bot. 2005, 56, 2153-2161. [CrossRef]

131. Gutiérrez-Luna, F.; López-Bucio, J.; Altamirano-Hernández, J.; Valencia-Cantero, E.; de la Cruz, H.; Macías-Rodríguez, L. Plant growth-promoting rhizobacteria modulate root-system architecture in Arabidopsis thaliana through volatile organic compound emission. Symbiosis 2010, 51, 75-83. [CrossRef]

132. Nassal, D.; Spohn, M.; Eltlbany, N.; Jacquiod, S.; Smalla, K.; Marhan, S.; Kandeler, E. Effects of phosphorus-mobilizing bacteria on tomato growth and soil microbial activity. Plant Soil 2018, 427, 17-37. [CrossRef]

133. Stephen, J.; Shabanamol, S.; Rishad, K.; Jisha, M. Growth enhancement of rice (Oryza sativa) by phosphate solubilizing Gluconacetobacter sp. (MTCC 8368) and Burkholderia sp. (MTCC 8369) under greenhouse conditions. 3 Biotech 2015, 5, 831-837. [CrossRef] [PubMed]

134. Brunner, S.; Goos, R.; Swenson, S.; Foster, S.; Schatz, B.; Lawley, Y.; Prischmann-Voldseth, D. Impact of nitrogen fixing and plant growth-promoting bacteria on a phloem-feeding soybean herbivore. Appl. Soil Ecol. 2015, 86, 71-81. [CrossRef]

135. Gopalakrishnan, S.; Srinivas, V.; Samineni, S. Nitrogen fixation, plant growth and yield enhancements by diazotrophic growthpromoting bacteria in two cultivars of chickpea (Cicer arietinum L.). Biocatal. Agric. Biotechnol. 2017, 11, 116-123. [CrossRef]

136. Navarro-Torre, S.; Barcia-Piedras, J.; Mateos-Naranjo, E.; Redondo-Gomez, S.; Camacho, M.; Caviedes, M.; Pajuelo, E.; RodríguezLlorente, I. Assessing the role of endophytic bacteria in the halophyte Arthrocnemum macrostachyum salt tolerance. Plant Biol. 2017, 19, 249-256. [CrossRef]

137. Pham Thi Thu, H.; Nguyen Thu, T.; Nguyen Dang Nguyen, T.; Le Minh, K.; Do Tan, K. Evaluate the effects of salt stress on physico-chemical characteristics in the germination of rice (Oryza sativa L.) in response to methyl salicylate (MeSA). Biocatal. Agric. Biotechnol. 2020, 23, 1-6. [CrossRef]

138. Dehnavi, A.; Zahedi, M.; Ludwiczak, A.; Perez, S.; Piernik, A. Effect of Salinity on Seed Germination and Seedling Development of Sorghum (Sorghum bicolor (L.) Moench) Genotypes. Agronomy 2020, 10, 859. [CrossRef]

139. Liu, T.; Li, R.; Jin, X.; Ding, J.; Zhu, X.; Sun, C.; Guo, W. Evaluation of Seed Emergence Uniformity of Mechanically Sown Wheat with UAV RGB Imagery. Remote Sens. 2017, 9, 1241. [CrossRef]

140. Dellaquila, A.; Spada, P. The effect of salinity stress upon protein synthesis of germinating wheat embryos. Ann. Bot. 1993, 72, 97-101. [CrossRef]

141. Fercha, A.; Capriotti, A.; Caruso, G.; Cavaliere, C.; Stampachiacchiere, S.; Chiozzi, R.; Laganà, A. Shotgun proteomic analysis of soybean embryonic axes during germination under salt stress. Proteomics 2016, 16, 1537-1546. [CrossRef] [PubMed] 
142. Zhang, C.; Luo, W.; Li, Y.; Zhang, X.; Bai, X.; Niu, Z.; Zhang, X.; Li, Z.; Wan, D. Transcriptomic Analysis of Seed Germination Under Salt Stress in Two Desert Sister Species (Populus euphratica and P. pruinosa). Front. Genet. 2019, 10. [CrossRef] [PubMed]

143. Liu, L.; Xia, W.; Li, H.; Zeng, H.; Wei, B.; Han, S.; Yin, C. Salinity Inhibits Rice Seed Germination by Reducing $\alpha$-Amylase Activity via Decreased Bioactive Gibberellin Content. Front. Plant Sci. 2018, 9, 275. [CrossRef]

144. Mahmood, A.; Turgay, O.; Farooq, M.; Hayat, R. Seed biopriming with plant growth promoting rhizobacteria: A review. FEMS Microbiol. Ecol. 2016, 92. [CrossRef]

145. Zulueta-Rodríguez, R.; Hernández-Montiel, L.; Murillo-Amador, B.; Rueda-Puente, E.; Capistrán, L.; Troyo-Diéguez, E.; CórdobaMatson, M. Effect of Hydropriming and Biopriming on Seed Germination and Growth of Two Mexican Fir Tree Species in Danger of Extinction. Forests 2015, 6, 3109-3122. [CrossRef]

146. Prasad, J.; Gupta, S.; Raghuwanshi, R. Screening Multifunctional Plant Growth Promoting Rhizobacteria Strains for Enhancing Seed Germination in Wheat (Triticum aestivum L.). Int. J. Agric. Res. 2017. [CrossRef]

147. Vaishnav, A.; Kumari, S.; Jain, S.; Varma, A.; Tuteja, N.; Choudhary, D. PGPR-mediated expression of salt tolerance gene in soybean through volatiles under sodium nitroprusside. J. Basic Microbiol. 2016, 56, 1274-1288. [CrossRef]

148. Sarropoulou, V.; Dimassi-Theriou, K.; Therios, I.; Koukourikou-Petridou, M. Melatonin enhances root regeneration, photosynthetic pigments, biomass, total carbohydrates and proline content in the cherry rootstock PHL-C (Prunus avium $\times$ Prunus cerasus). Plant Physiol. Biochem. 2012, 61, 162-168. [CrossRef]

149. Tal, O.; Haim, A.; Harel, O.; Gerchman, Y. Melatonin as an antioxidant and its semi-lunar rhythm in green macroalga Ulva sp. J. Exp. Bot. 2011, 62, 1903-1910. [CrossRef] [PubMed]

150. Uçarlı, C. Effects of Salinity on Seed Germination and Early Seedling Stage; IntechOpen: London, UK, 2020.

151. Halo, B.; Khan, A.; Waqas, M.; Al-Harrasi, A.; Hussain, J.; Ali, L.; Adnan, M.; Lee, I. Endophytic bacteria (Sphingomonas sp. LK11) and gibberellin can improve Solanum lycopersicum growth and oxidative stress under salinity. J. Plant Interact. 2015, 10, 117-125. [CrossRef]

152. Bharti, N.; Pandey, S.; Barnawal, D.; Patel, V.; Kalra, A. Plant growth promoting rhizobacteria Dietzia natronolimnaea modulates the expression of stress responsive genes providing protection of wheat from salinity stress. Sci. Rep. 2016, 6. [CrossRef]

153. El-Esawi, M.; Alaraidh, I.; Alsahli, A.; Alamri, S.; Ali, H.; Alayafi, A. Bacillus firmus (SW5) augments salt tolerance in soybean (Glycine max L.) by modulating root system architecture, antioxidant defense systems and stress-responsive genes expression. Plant Physiol. Biochem. 2018, 132, 375-384. [CrossRef]

154. Vimal, S.; Patel, V.; Singh, J. Plant growth promoting Curtobacterium albidum strain SRV4: An agriculturally important microbe to alleviate salinity stress in paddy plants. Ecol. Indic. 2019, 105, 553-562. [CrossRef]

155. El-Nahrawy, S.; Yassin, M. Response of Different Cultivars of Wheat Plants (Triticum aestivum L.) to Inoculation by Azotobacter sp. under Salinity Stress Conditions. J. Adv. Microbiol. 2020, 20, 60-79. [CrossRef]

156. Sun, L.; Lei, P.; Wang, Q.; Ma, J.; Zhan, Y.; Jiang, K.; Xu, Z.; Xu, H. The Endophyte Pantoea alhagi NX-11 Alleviates Salt Stress Damage to Rice Seedlings by Secreting Exopolysaccharides. Front. Microbiol. 2020, 10, 3112. [CrossRef]

157. Pinedo, I.; Ledger, T.; Greve, M.; Poupin, M. Burkholderia phytofirmans PsJN induces long-term metabolic and transcriptional changes involved in Arabidopsis thaliana salt tolerance. Front. Plant Sci. 2015, 6, 466. [CrossRef] [PubMed]

158. Manaf, H.; Zayed, M. Productivity of cowpea as affected by salt stress in presence of endomycorrhizae and Pseudomonas fluorescens. Ann. Agric. Sci. 2015, 60, 219-226. [CrossRef]

159. Khan, M.; Asaf, S.; Khan, A.; Ullah, I.; Ali, S.; Kang, S.; Lee, I. Alleviation of salt stress response in soybean plants with the endophytic bacterial isolate Curtobacterium sp. SAK1. Ann. Microbiol. 2019, 69, 797-808. [CrossRef]

160. Kharusi, L.; Yahyai, R.; Yaish, M. Antioxidant Response to Salinity in Salt-Tolerant and Salt-Susceptible Cultivars of Date Palm. Agriculture 2019, 9, 8. [CrossRef]

161. Ryu, C.-M.; Farag, M.; Hu, C.-H.; Reddy, M.; Kloepper, J.; Paré, P. Bacterial Volatiles Induce Systemic Resistance in Arabidopsis. Plant Physiol. 2004, 134, 1017-1026. [CrossRef] [PubMed]

162. Chen, L.; Liu, Y.; Wu, G.; Njeri, K.; Shen, Q.; Zhang, N.; Zhang, R. Induced maize salt tolerance by rhizosphere inoculation of Bacillus amyloliquefaciens SQR9. Physiol. Plant. 2016, 158, 34-44. [CrossRef] [PubMed]

163. Liu, H.; Wang, J.; Sun, H.; Han, X.; Peng, Y.; Liu, J.; Liu, K.; Ding, Y.; Wang, C.; Du, B. Transcriptome Profiles Reveal the Growth-Promoting Mechanisms of Paenibacillus polymyxa YC0136 on Tobacco (Nicotiana tabacum L.). Front. Microbiol. 2020, 11, 4174. [CrossRef]

164. Li, J.; Han, G.; Sun, C.; Sui, N. Research advances of MYB transcription factors in plant stress resistance and breeding. Plan Signal. Behav. 2019, 14, 1613131. [CrossRef]

165. Finatto, T.; Viana, V.; Woyann, L.; Busanello, C.; da Maia, L.; de Oliveira, A. Can WRKY transcription factors help plants to overcome environmental challenges? Genet. Mol. Biol. 2018, 41, 533-544. [CrossRef] [PubMed]

166. Qin, Y.; Tian, Y.; Liu, X. A wheat salinity-induced WRKY transcription factor TaWRKY93 confers multiple abiotic stress tolerance in Arabidopsis thaliana. Biochem. Biophys. Res. Commun. 2015, 464, 428-433. [CrossRef]

167. Yu, Y.; Wang, N.; Hu, R.; Xiang, F. Genome-wide identification of soybean WRKY transcription factors in response to salt stress. SpringerPlus 2016, 5. [CrossRef]

168. Wu, B.; Hu, Y.; Huo, P.; Zhang, Q.; Chen, X.; Zhang, Z. Transcriptome analysis of hexaploid hulless oat in response to salinity stress. PLoS ONE 2017, 12, e171451. [CrossRef] 
169. Thomas, J.; Kim, H.; Rahmatallah, Y.; Wiggins, G.; Yang, Q.; Singh, R.; Glazko, G.; Mukherjee, A. RNA-seq reveals differentially expressed genes in rice (Oryza sativa) roots during interactions with plant-growth promoting bacteria, Azospirillum brasilense. PLoS ONE 2019, 14, e217309. [CrossRef]

170. Malviya, M.; Solanki, M.; Singh, R.; Htun, R.; Singh, P.; Verma, K.; Yang, L.-T.; Li, Y.-R. Comparative analysis of sugarcane root transcriptome in response to the plant growth-promoting Burkholderia anthina MYSP113. PLoS ONE 2020, 15. [CrossRef] [PubMed]

171. Ho, W.; Hill, C.; Doblin, M.; Shelden, M.; van de Meene, A.; Rupasinghe, T.; Bacic, A.; Roessner, U. Integrative Multi-omics Analyses of Barley Rootzones under Salinity Stress Reveal Two Distinctive Salt Tolerance Mechanisms. Plant Commun. 2020, 1. [CrossRef] [PubMed]

172. Safdarian, M.; Askari, H.; Shariati, J.V.; Nematzadeh, G. Transcriptional responses of wheat roots inoculated with Arthrobacter nitroguajacolicus to salt stress. Sci. Rep. 2019, 9, 1792. [CrossRef] [PubMed]

173. Xie, S.; Wu, H.; Chen, L.; Zang, H.; Xie, Y.; Gao, X. Transcriptome profiling of Bacillus subtilis OKB105 in response to rice seedlings. BMC Microbiol. 2015, 15. [CrossRef] 